\title{
Collective Mechanical Behavior of Epithelial Cells - The Impact of Micro-Wounding
}

\author{
Dissertation \\ for the award of the degree \\ Doctor rerum naturalium \\ of the Georg-August Universität Göttingen \\ within the doctoral program \\ "International Max Planck Research School - \\ Physics of Biological and Complex Systems" \\ of the Georg-August University School of Science (GAUSS)
}

submitted by

Susanne Karsch

from Nordhorn

Göttingen, December 2018 


\title{
Thesis Committee:
}

First referee:

Second referee:

Third referee:

\section{Examination Board:}

First reviewer:

Second reviewer:

\section{Extended Examination Board:}

Prof. A. Janshoff

Institute of Physical Chemistry,

University of Göttingen

Prof. S. Köster

Institute for X-ray Physics,

University of Göttingen

Prof. J. Großhans

Institute of Developmental Biochemistry

University Medical Center Göttingen

Prof. A. Janshoff

Institute of Physical Chemistry,

University of Göttingen

Prof. J. Großhans

Institute of Developmental Biochemistry

University Medical Center Göttingen

\author{
Prof. S. Köster \\ Institute for X-ray Physics, \\ University of Göttingen \\ Dr. F. Rehfeldt \\ Third Institute of Physics - Biophysics \\ University of Göttingen \\ Prof. S. Rizzoli \\ Department of Neuro- and Sensory Physiology \\ University Medical Center Göttingen \\ Dr. T. Burg \\ Biological Micro- and Nanotechnology \\ Max Planck Institute for Biophysical Chemistry
}

Date of oral examination: 24th january 2019 
I, Susanne Karsch, hereby certify that my doctoral thesis entitled "Collective Mechanical Behavior of Epithelial Cells - The Impact of Micro-Wounding" has been written independently and with no other sources and aids than quoted.

Göttingen, 2018

Susanne Karsch 



\section{The Beauty of Biophysics Lies in the Beauty of Life}

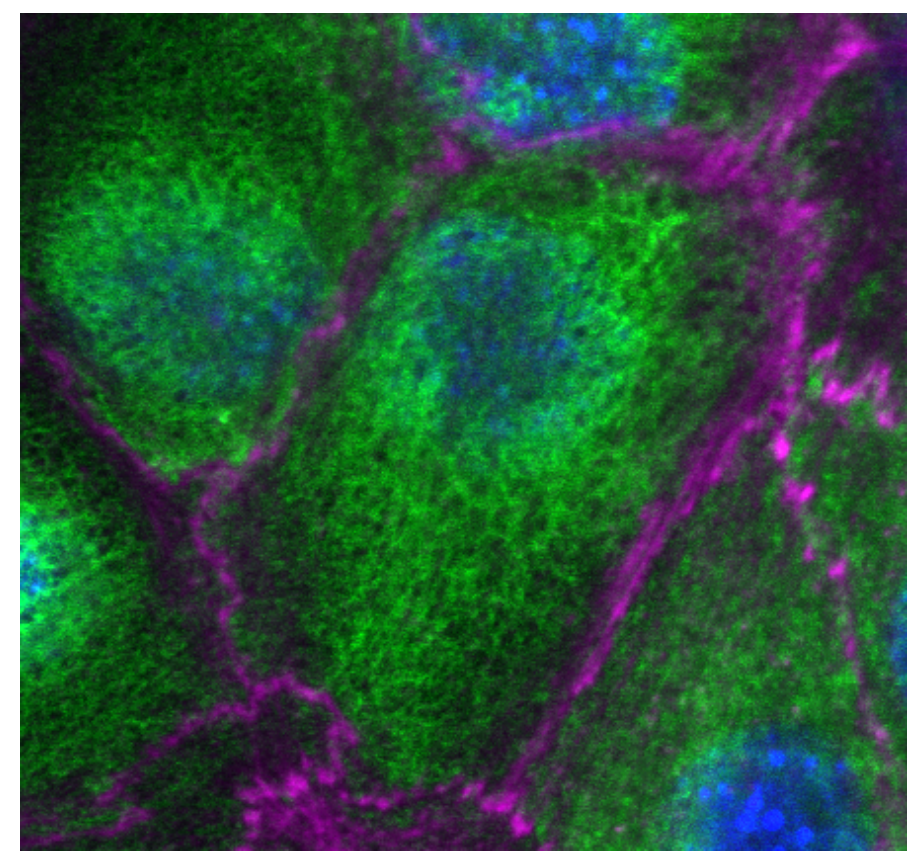

Keratinocytes stained for keratin (green), actin (magenta)

and the DNA (blue)

-To my family- 



\section{Abstract}

For epithelia, which are lining organs or blood vessels, the mechanical integrity of a whole cell sheet is indispensable. The question arises, how neighboring cells mechanically work together to build a functioning cell sheet. In order to address this question, epithelial cells were monitored during layer formation and critical culture conditions such as temperature to characterize the cell sheet behavior exposed to different environmental cues. To disturb cell sheet homeostasis, single cells were mechanically manipulated with a new micro-wounding technique which finally leads to a complete cell failure and subsequently a single-cell wound. Via optical microscopy the closure process was followed and an efficient purse string mechanism was found for MDCK II cells. The closure was accompanied by an increase in mechanical pretension, which was detected with atomic force microscopy. The increase in pretension was also found in cells which are not in direct contact with the wound but separated by up to three cell widths from the wounding site. This indicates that also small disturbances such as micro-wounding elicit a coordinated response in cell sheets, most probably mediated by cell junctions. Since the paradigm organ for wounding is the skin, keratinocyte cells were used for which a genetically modified knock out, lacking the entire keratin network, exists. In these knock out cells, junction localization and actin architecture was altered. Functional studies showed that differences in cell-cell coupling and barrier function, measured with electric cell-substrate impedance sensing, occur. Cells lacking the keratin network also showed a fragile layer integrity and an increase in wound size indicating an impaired response to micro-wounding. These changes were attributed to an altered mechanical homeostasis in individual cells and subsequently also the cell sheet.

In conclusion, this new approach of micro-wounding demonstrated that even single-cell wounds induce a collective mechanical response in epithelial cell ensembles and that wellcontrolled single-cell mechanical homeostasis is crucial for proper cell sheet function. 



\section{Contents}

1. Introduction 1

1.1. Epithelial cells . . . . . . . . . . . . . . . . . . . . . . 2

1.2. Contributors to cell mechanics . . . . . . . . . . . . . . . . . . . 4

1.2.1. The cell membrane . . . . . . . . . . . . . . . . . . . . . . 4

1.2.2. The cytoskeleton . . . . . . . . . . . . . . . . . . . . 4

1.2.3. Cell-cell and cell-matrix adhesions . . . . . . . . . . . . . . . 8

1.2.4. Early formation of epithelial cell-cell junctions . . . . . . . . . . 11

1.3. Cell lines . . . . . . . . . . . . . . . . . . . . . . . . . . . . . . . . . . 13

1.3.1. MDCK $\|$ cells . . . . . . . . . . . . . . . . . . . . . 13

1.3.2. Keratinocytes . . . . . . . . . . . . . . . . . 13

1.4. Mechanics and mechanosensing of a single cell . . . . . . . . . . . . . . 14

1.5. Collective mechanical behavior of epithelial cells . . . . . . . . . . . 18

1.6. Micro-wound healing mechanisms of epithelial cells . . . . . . . . . . . . 21

2. Materials and methods 25

2.1. Cell culture . . . . . . . . . . . . . . . . . . . . . . . . . . . 25

2.1.1. MDCK $\|$ cells . . . . . . . . . . . . . . . . . . . 25

2.1.2. Keratinocytes . . . . . . . . . . . . . . . . . . . 25

2.1.3. Culture conditions during the experiments . . . . . . . . . . 2 26

2.1.4. Transfection of E-Cadherin-GFP cells . . . . . . . . . . . . . . . 27

2.2. Wounding strategies for a confluent cell layer . . . . . . . . . . . . . . . 28

2.2.1. Induction of large wounds . . . . . . . . . . . . . . . . . . . 28

2.2.2. Induction of single-cell defects . . . . . . . . . . . . . . . . . . . . 29

2.3. Light microscopy techniques . . . . . . . . . . . . . . . . . . . . . . . . 30

2.3.1. Cell labeling . . . . . . . . . . . . . . . . . . . . . . . . 30

2.3.2. Chemicals used during cell experiments . . . . . . . . . . . . . . 31

iii 
2.3.3. Video and image acquisition . . . . . . . . . . . . . . . . . 32

2.3.4. Video and image processing . . . . . . . . . . . . . . . . 33

2.3.5. Cell labeling and image acquisition protocol for keratinocyte ex-

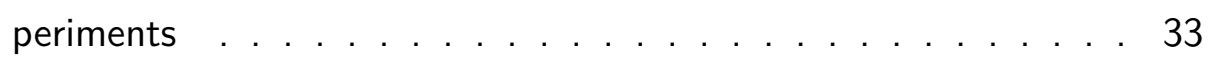

2.4. Patterning of confluent cell layers . . . . . . . . . . . . . . 35

2.5. Atomic force microscopy . . . . . . . . . . . . . . . . . . . 36

2.5.1. Basic setup of an AFM . . . . . . . . . . . . . . 36

2.5.2. Tension model . . . . . . . . . . . . . . . . . 37

2.5.3. Force curve recording and processing . . . . . . . . . . . . . . 40

2.5.4. Topography scans . . . . . . . . . . . . . . . . . . . . . 41

2.6. Electric cell-substrate impedance sensing . . . . . . . . . . . . . . . 41

2.7. Cell adhesion noise spectroscopy . . . . . . . . . . . . . . . . . . . . . 42

3. Results 45

3.1. Impact of culture conditions on MDCK II cells . . . . . . . . . . . . 45

3.1.1. Culture medium as critical factor . . . . . . . . . . . . . . . . . 45

3.1.2. Impact of light exposure during live experiments . . . . . . . . . 46

3.1.3. Temperature dependent behavior of MDCK II cells . . . . . . . . 47

3.2. Growth and onset of collective behavior of MDCK II cell layers . . . . . . 52

3.2.1. Growth behavior of a MDCK II cell layer . . . . . . . . . . . . . 52

3.2.2. Early cell-cell junction formation in a growing cell layer . . . . . . 53

3.2.3. Controlling cell-cell junction geometry using micro patterning . . 57

3.3. Classical wound healing essays to create large wounds . . . . . . . . . . 58

3.4. Single-cell defects in a confluent MDCK II layer . . . . . . . . . . . . . . 61

3.4.1. Characteristic closure of a single-cell defect . . . . . . . . . . . . 61

3.4.2. Mechanical properties of cells neighboring the single-cell defect . 63

3.4.3. Mechanical properties of cells neighboring larger wounds . . . . . 65

3.4.4. Spatial extent of altered mechanics in the wounded cell layer. . . 65

3.4.5. Constant cell geometries during closure . . . . . . . . . . . . . . 67

3.4.6. Contributors to the collective closure . . . . . . . . . . . . . . 68 
3.5. Influence of keratin deficiency on barrier function and mechanical integrity in keratinocyte cell sheets . . . . . . . . . . . . . . . . . . 73

3.5.1. Absence of the keratin network leads to altered junction and cytoskeletal architecture . . . . . . . . . . . . 73

3.5.2. Barrier function is impaired in keratin lacking epidermal cell sheets 76

3.5.3. Altered single cell mechanics and inefficient wound closure in keratin lacking cells . . . . . . . . . . . . . . . . . . 78

3.5.4. Loss of mechanical coupling in keratin lacking epidermal sheets . 80

3.5.5. Insertion of K14 rescues phenotype but not the full functionality. 82

4. Discussion

4.1. Importance of culture conditions on cell morphology . . . . . . . . . . . 87

4.2. Junction establishment and onset of collective behavior during layer growth 90

4.3. Classical large-scale wound healing assays . . . . . . . . . . . . . . . . 91

4.4. Effects of single-cell defects in MDCK II layers . . . . . . . . . . . . . . 92

4.5. Impact of keratin deficiency on mechanical homeostasis in keratinocyte cell sheets . . . . . . . . . . . . . . . . . . . . . . . 94

$\begin{array}{lr}\text { 5. Conclusion } & 99\end{array}$

\begin{tabular}{lr}
\hline A. Appendix & 129
\end{tabular} 



\section{Introduction}

From a biological point of view the human body is a huge accumulation of cells. All types of cells have to work together to form a functioning body.

The largest building blocks of an organism are the organs which fulfill specific tasks in the organism (figure 1.1). The skin, for example, has an important barrier function for the organism to block pathogens or external chemicals to enter the organism. Organs itself consist of several types of tissues. To build for example the skin, tissues as the epidermis or dermis are stacked on top of each other and have to function together. Zooming further in, the epidermis consists of cells, mostly keratinocytes [1]. All these building blocks on different length scales have to contribute to the functionality of the larger structures by building cooperative collectives. Especially for the skin, mechanical properties such as elasticity are important to allow body movements but at the same time the barrier must maintain. In order to achieve this goal, the whole organ has to act as a collective entity but also every smaller building block, as the tissue or even single cells, have to fulfill specific mechanical requirements. A loss of homeostasis on any of these levels can lead to severe diseases as, e.g., cancer, where cell growth becomes abnormal, invasiveness increases and the mechanical phenotype changes [2]. Additionally, this homeostasis can be disturbed by external stimuli, as for example macroscopic wounding. Mechanisms must exist, which deal with this perturbance. In healthy organisms a pre-defined signal cascade starts after wounding, which involves several cell types and signaling pathways in order to close the wound and gain homeostasis again [3].

In this study the events that take place after wounding shall be investigated via examining epithelial cells which line surfaces and cavities and hence form barriers in an organism. The method of in vitro wounding of cell cultures allows to monitor how these cells react to a small disturbance and regain homeostasis again. A deeper understanding on the cellular and tissue level will help to understand how the small building blocks have to work together to build a functioning larger collective. 


\section{Introduction}

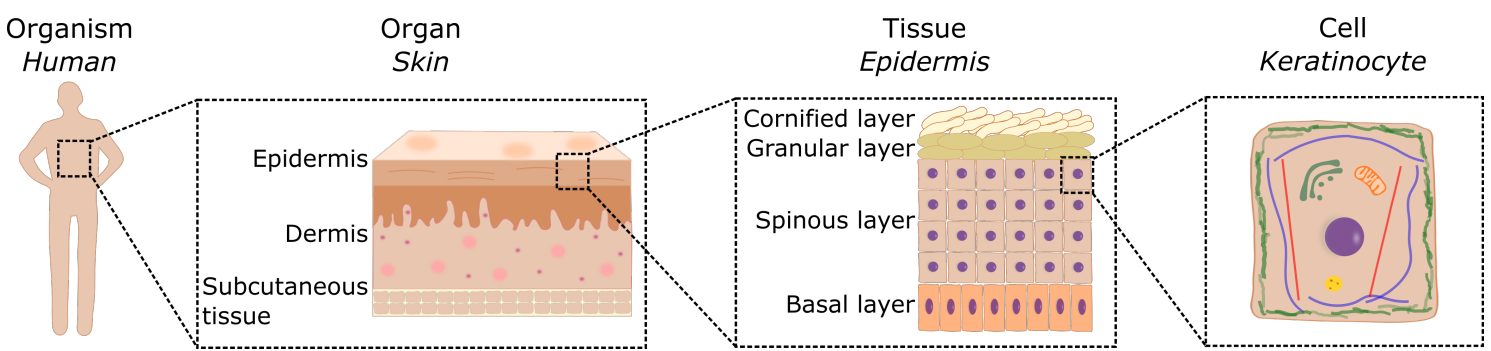

Figure 1.1.: To ensure a functioning organism, subunits on several length scales have to work properly together. The biggest organ in the human body is the skin [1], consisting of several types of tissues such as for example the dermis and the epidermis. The most outer tissue, the epidermis, itself consists of several layers as the basal layer or the spinous layer, where mostly keratinocytes as the main cell type can be found.

\subsection{Epithelial cells}

The human body consists of diverse types of cells, as for example, muscle cells, epithelial cells or nerve cells. They all developed from a pluripotent embryonic stem cell through the differentiation process and became specific in shape and function. Furthermore, almost all the cells share the same organelles and structural elements which can be found in figure 1.2 and will be shortly introduced:

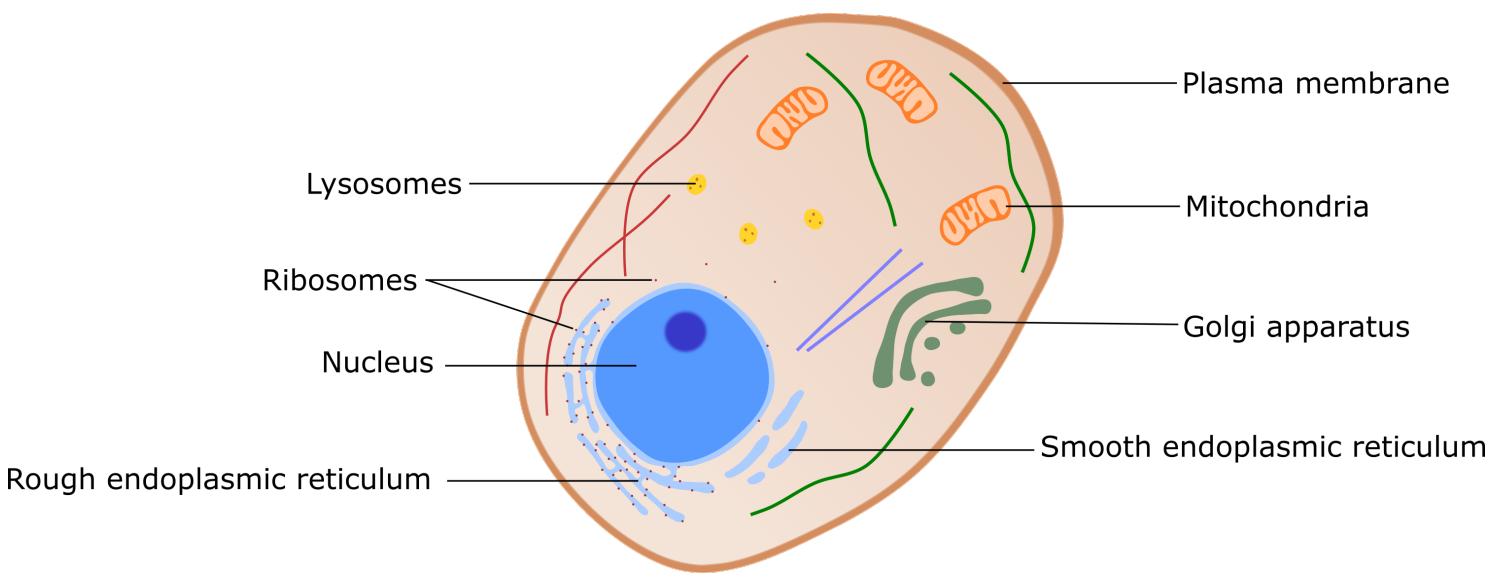

Figure 1.2.: An eukaryotic cell. Eukaryotic cells are surrounded by a membrane build up from a lipid bilayer. Inside the cell, the nucleus contains the DNA and the nucleus surrounding membrane is continuous with the endoplasmic reticulum (ER). The rough ER is covered with protein synthesizing ribosomes while the smooth is not. The golgi apparatus allows vesicle formation for transport processes and lysosomes are specific vesicles for protein digestion. Mitochondria are major players in energy supply for chemical processes inside the cell. Cytoskeletal components are only adumbrated and will be explained in more detail in section 1.2 .2 . 
Central for these cells is the surrounding plasma membrane which is not as rigid as cell walls in plant cells but effectively separates the cytoplasm from the extracellular surrounding. To fully maintain cell stability, the cytoskeleton comes into play which will be explained in more detail in section 1.2.2. For energy supply, membrane surrounded mitochondria exist while the endoplasmic reticulum (ER) is important for protein synthesis and transport. The rough endoplasmic reticulum is studded with ribosomes, which are major sites for protein synthesis. Another organelle, the golgi apparatus, engulfs proteins received from the ER with membrane vesicles to allow transport throughout the cell. Protein digestion and organelle degradation takes place in lysosomes which contain specific enzymes for this task. The cells nucleus is involved in the differentiation process and cell division as it contains the genetic material, the deoxyribonucleic acid (DNA). This organelle is separated as well by a membrane system, the nuclear envelope. More detailed information on general cell structure and function can be found in prominent biology text books as [4].

Considering different types of cells especially in vertebrates, epithelial cells are a very pronounced cell type. More than $60 \%$ of the cells in these organisms are epithelial cells [5]. Epithelial cells maintain chemical gradients within an organism and separate liquids from neighboring compartments. Furthermore, they provide the mechanical stability of this barrier. Epithelial cells can be found throughout the whole body and can adapt different shapes depending on the location (see figure 1.3). However, all monolayered epithelia have in common that they are highly axially polarized and have an apical surface covered with microvilli. Microvilli are membrane protrusions filled with cytoskeletal structures which increase the membrane surface area allowing increased nutrient absorption or chemical secretion. Laterally, epithelial cells are tightly connected to the neighboring cells via cell-cell-junctions (see section 1.2.3). This tight coupling is crucial for efficient barrier formation. At the bottom of the cell, the basal side, epithelial cells are connected to the extracellular matrix, the basal lamina. 


\section{Introduction}

A

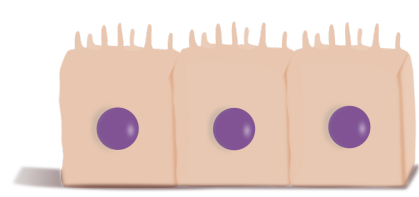

B

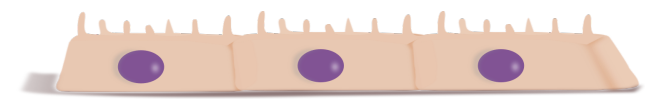

C

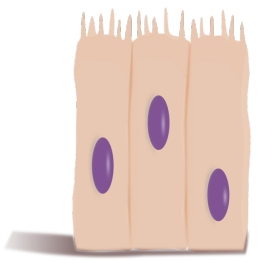

Figure 1.3.: Geometry of epithelial cells. Monolayered epithelial cells, called simple epithelium, adapt different morphologies depending on their location in the body. (A) Cuboidal cells can be found in the kidney, (B) squamous cells in the lung alveoli and (C) columnar cells for example in the intestine.

\subsection{Contributors to cell mechanics}

\subsubsection{The cell membrane}

The cell membrane consists of a lipid bilayer with a hydrophobic core in the middle of the bilayer and hence acts as a diffusion barrier. The average thickness of a membrane is $5 \mathrm{~nm}$ [5]. Still, the lipid and protein composition can be very diverse depending on the place and function inside a cell or the actual cell type [6]. Furthermore, the architecture especially the packing parameter of the individual lipids determines the curvature and hence the overall shape of the membrane [7]. In general, the outer cell membrane is supported by the underlying cytoskeleton building a composite two dimensional solid sheet. This not only limits diffusion inside the membrane but the composite creates an overall cell tension comparable to a surface tension in liquid droplets [8]. The tension regulation has been proven to be a major factor in many cell processes such as endocytosis rates during mitosis [9] or lamellipodia extension [10]. Only during tether or bleb formation, where hollow evaginations without cytoskeletal support form, tension of the membrane alone can be estimated [11].

\subsubsection{The cytoskeleton}

The cytoskeleton is an integral part inside all animal cells. As mentioned above, animal cells do not have a cell wall to give mechanical stability and the outer cell membrane without support also lacks this property. Hence structures inside the cell have to build a scaffold supporting the fluid-like membrane and dictating the cell shape. Functions as spatial organization inside the cell, physical and biochemical connection to the surrounding cells and coordinated force generation are carried out by the cytoskeleton [12]. 
The cytoskeleton consists of three different types of filaments, namely actin filaments, intermediate filaments (IFs) and microtubules (MTs) (figure 1.4), having different intrinsic properties which will be introduced in the following. Here, more structural and biological aspects will be in focus while their contributions to single and collective cell mechanics will be dealt with in section 1.4 and 1.5 .
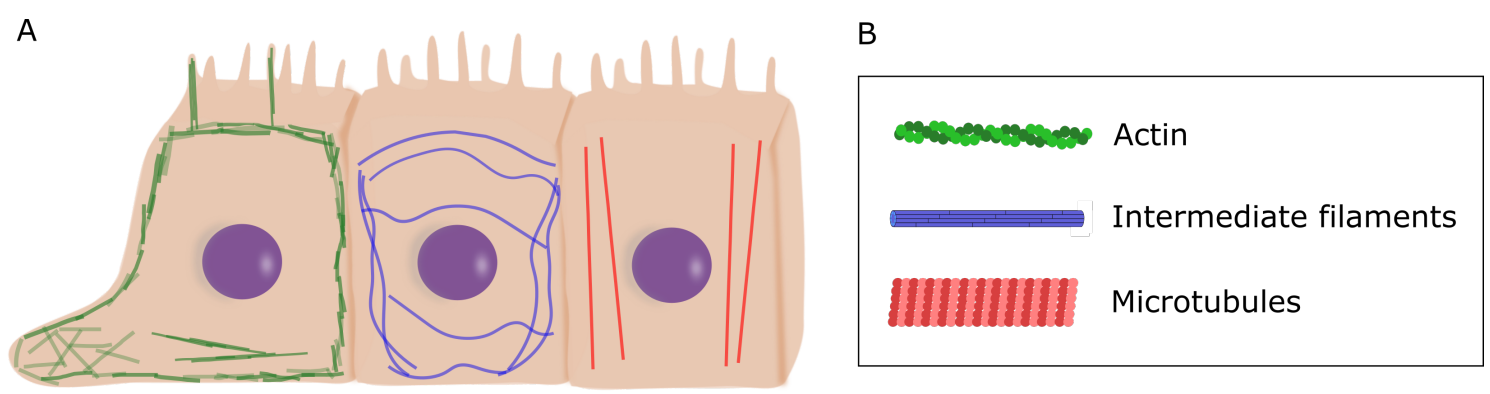

Figure 1.4.: Cytoskeletal filaments inside the cell. (A) The cytoskeleton is the scaffold for the cell which can be found at different locations inside the cell and in different shapes. Actin filaments (green) build the cells cortex underneath the membrane and span lamellipodia in a meshwork like structure. Apical in microvilli and basal in stress fibers actin filaments are aligned parallel to each other. Intermediate filaments (blue) build a highly motile and flexible network spanning the whole cell. Microtubules (red) are stiff rods oriented vertically inside the cell. (B) All three types of filaments show different filament architecture. While actin consists of one type of globular monomers building two strands twisted around each other, intermediate filaments more show a rodlike structure packed together from several elongated subunits. Microtubules build hollow rods consisting of two different types of monomers arranged in 13 distinct lines called protofilaments.

\section{Actin filaments}

Actin filaments, also called microfilaments, are the major player for exerting forces inside the cell and also macroscopically in muscle contraction. They consist of globular $42 \mathrm{kDa}$ actin monomers which measure $6 \mu \mathrm{m}$ in diameter and have an intrinsic polarity [13]. Therefore, also the polymer has distinct ends with $\approx 10$ times larger association rate at the barbed end (plus end) than at the pointed end (minus end) [14]. Furthermore, the polymerization rates are ATP-dependent [15]. Through polymerizatian, a doublestranded helical structure with a diameter of 5-9 $\mathrm{nm}$ is build [5]. In vitro filament length of up to $60 \mu \mathrm{m}$ can be reached but the average length lies around $\mathrm{L}=20 \mu \mathrm{m}[16]$. In vivo mostly smaller length of a few $\mu \mathrm{m}$ are present [17]. Looking at filament mechanics, these filaments have a persistence length of about $L_{p}=15 \mu \mathrm{m}$ and hence behave like a semi-flexible polymer [18]. 


\section{Introduction}

In cells, these filaments are highly crosslinked and build different types of network structures depending on the position inside the cell. The crosslinking via special proteins is either passive as in case of filamin or $\alpha$-actinin or can be active as in the case of myosin motors. Here, under consumption of ATP, conformational changes inside individual myosin molecules enable the motor to walk across actin filaments [19]. Due to the alignment of lots of motors and actin filaments, the filaments get sheared with respect to each other and contractile forces can be exerted within the actin structures. Several other actin binding proteins exist which induce branching, severing or capping of filaments [20]. Further actin binding proteins which link actin filaments to other cell structures were found as well: for example ezrin couples actin to the membrane [21] while plakins link actin to other cytoskeletal filaments [22]. These diverse dynamics and interactions make actin an important constituent in a lot of physiological processes.

As mentioned above, different types of actin structures exist at different locations inside the cell. In the actin cortex being located underneath the plasma membrane, the actin builds an isotropic and highly crosslinked network with a meshsize ranging from 20-250 nm and a thickness of 50-100 nm [23. As explained above, this network is connected to the plasma membrane being especially important for tension regulation inside cells [24].

Basal, actin fibers mainly form thick fibers. The individual filaments are bundled and thereby build so called stress fibers [20]. Here, myosin is highly active making the stress fibers powerful contractile structures being important for physically sensing the environment via focal adhesions (further information in section 1.4).

For migrating cells, lamellipodia and filopodia formation are crucial. Filopodia are fingerlike protrusions where the actin is aligned with the barbed end to the front to probe the surrounding in front of the cell. Lamellipodia consist of branched actin networks where the actin related protein $2 / 3$ (Arp2/3) complex is mainly responsible for structure determination. Lamellipodia build up at the leading edge of the cell, dragging the cell body along [20].

\section{Intermediate filaments}

There exist 5 major subtypes of intermediate filaments (IFs). In epithelial tissue, mainly type I and II keratins are found in the cytoplasm while in the nucleus type V IFs called 
lamins are present [25]. All types show a unique polymerization pattern. The monomer has an extended central $\alpha$-helical rod domain flanked by specific $\mathrm{N}$ - and C-termini. The monomers self-assemble into coiled-coil dimers, mostly heterodimers. These dimers antiparallely align with another dimer to form a tetramer which is not polar. Eight tetramers align next to each other to form unit length filaments with a length of about $65 \mathrm{~nm}$ [26]. Linear alignment of these filaments allows to elongate the whole filament which has a final diameter of about $10 \mathrm{~nm}[25]$.

IFs have a smaller persistence length $\left(L_{p} \leqslant 2 \mu \mathrm{m}\right)$ than actin [26]. Their filament length can reach up to $20 \mu \mathrm{m}$ [17] being on the order of typical cell sizes. Due to the small persistence length, they mostly adapt a buckled contour. Similar to actin bundling proteins, also bundling proteins for intermediate filaments exist such as filaggrin or plectin [5]. In terms of cytoskeletal features, intermediate filaments build up dense network structures where filament assembly starts in the cell periphery and filament integration and bundling enhances during inward movement. In the center of the cell, a mature network is formed around the nucleus as a perinuclear cage and partial disassembly of filaments takes place [27].

\section{Microtubules}

Microtubules (MTs) are the stiffest of the cytoskeletal filaments. They have a persistence length of $L_{p}=1000-5000 \mu \mathrm{m}$ forming a stiff rod inside a cell and reaching length of 5-15 $\mu \mathrm{m}$ [17]. They consist of $\alpha$ - and $\beta$-tubulin which form a heterodimer as subunit. These subunits can assemble into hollow tubes with 13 parallel protofilaments. Because of the heterogeneous subunit, also MTs have a polarization with the fast growing plus end consisting of $\beta$-tubulin and the minus end of $\alpha$-tubulin [5].

MTs are important for trafficking and protein transport inside the cell [5]. Due to the intrinsic polarization, molecular motors being packed with a specific cargo can move uniformly along the filaments and use them as tracks, mostly to the outer part of the cell. Additionally, MTs build up the mitotic spindle which separates the chromosomes during cell division [5]. Also for MTs, associated proteins exist which can induce bundling and cross linking [22]. 


\section{Introduction}

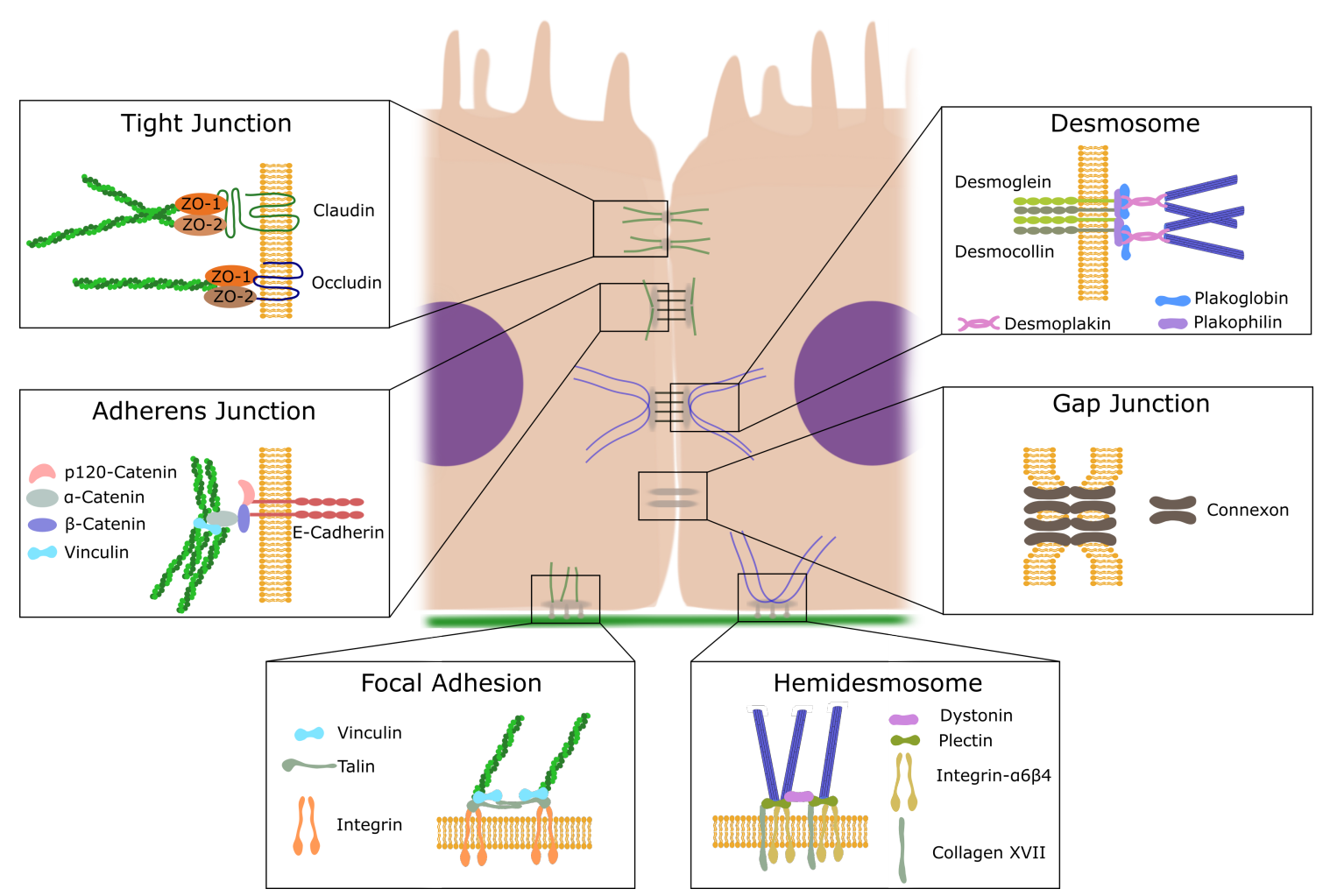

Figure 1.5.: Junctions at the cells' periphery. The cell is connected to neighboring cells and the underlying matrix. Most apical, tight junctions consisting of ZO-1 (zonula occludens-1), ZO-2 and transmembrane proteins as claudin and occludin are present and regulated by actin fibers. Underneath adherens junctions formed by E-cadherin, several catenins and vinculin connect mechanically the actin cytoskeleton of neighboring cells. Intermediate filaments are anchored via desmosomes (consisting cytoplasmically of plakoglobin, plakophilin and desmoplakin as well as desmocollin and desmoglein as transmembrane proteins) to their neighbors. Located lateral basal, gap junctions allow chemical transport between cells via connexons. Anchors to the underlying matrix are either focal adhesions or hemidesmosomes containing different integrin isoforms as transmembrane proteins. Either vinculin and talin or plectin and dystonin are members of the cytoplasmic plaque in focal adhesions and hemidesmosomes, respectively. Note, that the junctions mostly consist of far more proteins than shown in this figure, however for clarity only the most prominent ones are depicted here. Subfigures adapted from [5].

\subsubsection{Cell-cell and cell-matrix adhesions}

In vivo, the cell is not an isolated object but highly interconnected with other neighboring cells and also the surrounding environment (figure 1.5). Different multi protein complexes allow signaling to and sensing from the outside and contribute to different tasks. The composition of all the protein complexes and their biological relevance will be outlined in the following while detailed contributions to mechanical aspects follow in sections 1.4 
and 1.5 .

\section{Gap junctions}

Gap junctions allow the cell-to-cell transmission of molecules [28]. At these sites, the two membranes of neighboring cells come in close contact and the small gap in between is bridged mostly by the transmembrane protein connexin [5]. Connexins therefore form a direct passageway between the two cytoplasms allowing inorganic ions and small watersoluble proteins to diffuse directly from one cell to another.

\section{Tight junctions}

Tight junctions are important for the formation of diffusion barriers and thus are crucial for epithelial cell sheets. They are able to regulate the paracellular transport of water and can be found at the apical side of epithelial cells. Tight junctions consist of several proteins being either transmembrane such as claudin or occludin [29] or parts of the cytoplasmic plaque like ZO-1 (zonula occludens-1) [30]. The extracellular domains of transmembrane proteins of two adjacent cells build a tight seal holding the two cell membranes in close proximity [31]. For the maintenance of this binding calcium needs to be present. Tight junction complexes form rings completely encircling the cells [31]. Besides their function as diffusion barriers, they are also important for establishment and maintenance of cell polarity [32]. Due to this ring structure, they divide the membrane in an apical and a lateral/basal part which can contain different transmembrane proteins and lipid mixtures.

In addition, there is also evidence that tight junctions are connected to cytoskeletal components like actin which can actively regulate the diffusion barrier through actomyosin contraction [33].

\section{Adherens junctions}

Adherens junctions directly connect actin cytoskeletons of neighboring cells via a multi protein aggregate.

The transmembrane protein is E-cadherin where the extracellular part consists of five repeating domains E1 - E5 which can bind to opposing E-cadherin in a trans-interaction [34]. As for all classical cadherins, the homophilic binding is calcium dependent [35]. 


\section{Introduction}

Inside the cytoplasm, E-cadherin is bound to p120-catenin, which further connects to $\beta$-catenin and $\alpha$-catenin which is finally connected to the actin cytoskeleton. In addition, several other proteins can be found in this protein plaque [36]. Importantly, $\alpha$-catenin can change its conformation to an open state being stabilized by vinculin, which then provides more actin binding sites and strengthens the cytoskeletal connections [37]. Additionally, adherens junctions dynamically rearrange and build different structures with the cytoskeleton. On the one hand, they can be either assembled in small spots as punctate junctions where the actin filaments perpendicularly terminate at the junctions, hence contraction acts perpendicular to the cell membrane which is discussed to be important in wound closure [38, 39]. On the other hand, complexes are known where actin filaments are connected parallel to larger adherens junction complexes. These regions normally form circumferential belts underneath the tight junctions around cells and are known as zonula adherens [40]. Dynamical rearrangement and remodelling of these complexes is highly important for shape changes and movement in epithelial tissue [41].

\section{Desmosomes and hemidesmosomes}

Desmosomes and hemidesmosomes are anchoring junctions connected to the IF network. Desmosome transmembrane proteins are desmoglein and desmocollin which belong to the cadherin superfamily [42]. Inside the cell, the protein plaque mostly consists of plakoglobin, plakophilin and desmoplakin which further connects to IFs [43]. Desmosomes are adhesion complexes at the lateral membrane site between two neighboring cells whereas hemidesmosomes basally connect the IF filament network of one cell to the underlying matrix, the basement membrane. Furthermore, hemidesmosomes show a different protein architecture than desmosomes in which integrin heterodimers couple the membrane to the extracellular matrix (ECM). Inside the cell, plakin proteins such as plectin and bullous pemphigoid antigens build the protein plaque bridging integrin and the IF network [44].

Desmosomal adhesion is calcium dependent. However, it was shown that during maturation a hyperadhesive state can be reached [45]. In this state, the adhesion strength is higher and depletion of calcium does not weaken the interaction. This switch to a hyperadhesive state is reversible which is important e.g. in reepitheliazation during 
wound healing where migration of epithelial cells and hence weak cell-cell interactions are fundamental [46].

\section{Focal adhesions}

Focal adhesions (FAs) are the cells' anchor to connect the actin cytoskeleton to the ECM. At focal adhesions, integrins are embedded in the membrane which provide binding sites for ECM proteins. FAs show a complex protein structure with over 50 reported associated proteins [47]. Important proteins inside the cellular adhesion plaque are paxillin, talin and also vinculin [48]. In a force dependent manner, they rearrange or change conformation in order to build stable connections to actin stress fibers at the basal side [49]. During migration the dynamic establishment and rearrangement of focal adhesion spots is essential [50]. Focal adhesions are known to be sensors of external forces and force transducers inside the cell [51] which will be further introduced in section 1.4 .

\subsubsection{Early formation of epithelial cell-cell junctions}

Different types of junctions serve different purposes and all of them are necessary for proper tissue function. Especially the barrier formation and induction of cell polarity are necessary from early on. Hence, it is not surprising that also junction assembly takes place in a coordinated way. Proper protein recruitment in early stages is a key to junction formation.

Upon early contact of two neighboring lamellipodia, E-cadherin and $\alpha$-catenin are recruited to the site of contact. This induces local actin polymerization prior to the actin remodeling and formation of the peripheral actin belt [52]. Studies showed, that cis interactions between cadherins are not necessary for adherens junctions formation but that the formation of cadherin nanoclusters increases the anchoring of actin [53]. Moreover, independently of existing cell-cell contact, low-density E-cadherin nanoclusters are present in the cell membrane and are delimited by actin [54]. However, for initial cellcell contact and the later formation of tight junctions, E-cadherin trans interactions are crucial [55, 56]. In calcium switch experiments, ZO-1 can be found in the initial adhesion spots as well due to its binding to $\alpha$-catenin [57]. Only at later stages the proper formation of tight junction complexes including ZO-1 at the apical side of cells can be observed [57]. Capaldo showed that depletion of $\alpha$-catenin causes long-term disruption 


\section{Introduction}

of tight junctions ( $\mathrm{TJs}$ ) while depletion of E-cadherin only delays the formation of $\mathrm{TJ}$ complexes but does not disrupt established junctions [58].

Going to larger structures, the positioning of TJs within a multilayered tissue is determined by E-cadherin-induced signaling [59] highlighting the intertwined regulation of the junctions.

For desmosome formation, individual proteins are transported via vesicles in distinct stages to the plasma membrane [60]. Stabilization through linkage to intermediate filaments then leads to the formation of a desmosomal plaque [52]. Also for desmosome formation, a contribution of E-cadherin was found: in early studies it was shown that blocking E-cadherin delays desmosome formation [56] and in recent studies it was found that an interaction between desmoglein and E-cadherin facilitates desmosome assembly [61]. However, desmosomal cadherin in combination with only plakoglobin and desmoplakin is already sufficient to form a desmosomal plaque [60].

All these studied showed that on the protein level, E-cadherin is a major player in triggering very early cell-cell adhesion as it also interferes with desmosome and tight junction formation.

Upon layer growth, mature adhesions form. The exact time-dependent growth behavior depends on several factors such as culture surface [62] or seeding density. During maturation, actin and cell junctions rearrange with a mechanism of combined adhesion and compaction to form a confluent layer as shown with a fluorescently tagged E-cadherin protein [63]. This regulation and establishment of physical contact between cells is a major event in collective behavior emergence.

\subsection{Cell lines}

\subsubsection{MDCK II cells}

Madin-Darby Canine Kidney (MDCK) cells were derived from the kidney of an adult female cocker spaniel in 1958 by Madin and Darby. This cell line was firstly characterised by Gaush in 1966 [64]. The parental cell line showed a lot of heterogeneity [65] so subtypes were isolated. These nowadays available strains show different characteristics and should not be confounded [66]. In this study only MDCK II cells were used which stem from one of these subclones. Together with other strains, they serve as a widely used 
model for epithelial cell polarity and cell junction studies [67, 68]. In addition, several aspects of collective phenomena are already studied with MDCK II cells as e.g. migration behavior [69, 70] or growth regimes on elastic substrates [62]. A detailed literature review of these collective phenomena follows in section 1.5 .

\subsubsection{Keratinocytes}

The second cell line used in this thesis are primary murine keratinocytes isolated from the epidermis of mouse embryos. Also, a knock out mouse strain was generated in which the type I keratin gene cluster was deleted [71] (called $\mathrm{Kty}^{-/-}$). This resulted in a complete loss of keratin filaments in the epidermal cells [71, 72]. Moreover, lentiviral transduction was used to rescue the knock out cells and reexpress keratin 14 in the cells [72]. Thereby, three different types of structural different cells are accessible which allow to dissect keratin contributions to the cells collective mechanical behavior.

Keratinocytes are the most prominent cell type in the epidermis [1] and build a multilayered structure (see figure 1.1). Cells in a proliferative state can be found in the basal layer which is in direct contact with the basal lamina. During differentiation they traverse the spinous and the granular layer until they end up in the most outer cornified layer as dead cells. During this process, the keratinocytes express different protein patterns. In early differentiation (basal keratinocytes) they mostly contain the keratin pair $\mathrm{K} 5 / \mathrm{K} 14$. In later stages $\mathrm{K} 1 / \mathrm{K} 10$ gene expression is dominant [73]. In addition, protein composition of the desmosomes changes during this process [43].

The epidermis as the most outer layer of human skin is the first structure being affected upon macroscopic wounding. In the first stage of wound healing, platelets build a plaque to cover open injuries. Later on keratinocytes are major players and wander into the wounded area which is accompanied by upregulation of specific keratin isoforms [3, 74] (detailed information in section 1.6).

\subsection{Mechanics and mechanosensing of a single cell}

Cells can on the one hand sense and respond to mechanical cues from the outside but on the other hand also change their internal mechanics and apply contractile forces to the surrounding. 


\section{Introduction}

Concerning extracellular mechanical cues, it was shown that e.g. stem cells express different lineage markers after being placed on substrates with different elasticities [75]. These markers were typical for cells which are exposed to the same elasticity regime in their native environment as of the substrate. Furthermore, a phenomenon called durotaxis is known which describes the matrix stiffness guided migration behavior of cells [76]. These two phenomena can be explained by the cells' ability to sense the surrounding via the adhesion spots to the substrate. So, the mechanical cue at the focal adhesion starts biochemical and biophysical processes inside the cell known as mechanotransduction [77].

Evidence is arising that also cadherin-mediated junctions function in a similar manner but this time mechanical signals from neighboring cells are sensed at the adhesion sites [78]. In the following, the aim is to shine light on how cell structures like adhesion sites and the cytoskeleton respond to these external stimuli and define the cells mechanics (figure 1.6).

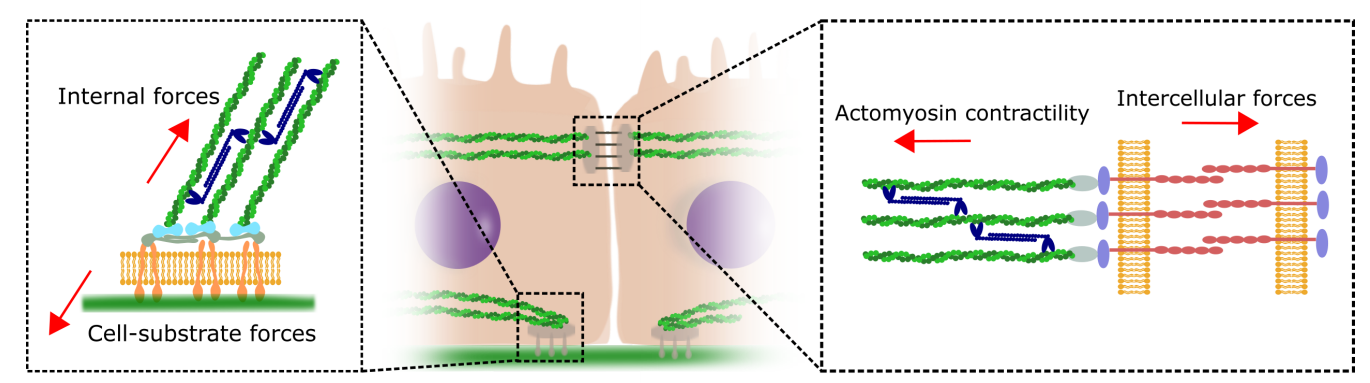

Figure 1.6.: Mechanosensing of cells. Major contributors to mechanosensing are actin coupled adhesions. At adherens junctions intercellular forces are applied and transmitted while at focal adhesions cellsubstrate forces arise. Internal forces due to myosin (blue) moving along actin filaments lead to internal contraction of the cell which is counterbalanced at the junctions. Thereby, cell junctions are the first stages of mechanosensing while the cytoskeleton is also affected by external cues and determines the overall cell mechanics.

\section{Adhesion sites}

The direct contact of a single cell to its surroundings are the adhesion sites and their protein complexes as described above. These multiprotein aggregates are the first step in force sensing and transmission. Via microscopy images it can be shown that for example adherens junctions or focal adhesions differ in size and distribution depending on 
their surrounding and their maturation state [54, 79]. Application of mechanical forces can regulate the size of the junctions [80]. Via novel synthesized Förster resonance energy transfer (FRET) sensors being integrated in the adhesion molecules it is possible to measure the forces acting within junctional proteins. Forces of $2.5 \mathrm{pN}$ can be found for vinculin in stable focal adhesions [81] and forces in the same range were also found for the cytoplasmic domain of E-cadherin [82]. One important aspect in this discussion is the interaction of the anchoring proteins to the cytoskeleton. In adherens junctions and also focal adhesions, specific proteins can undergo conformational changes and by this expose new binding sites and foster the adhesion as e.g. $\alpha$-catenin, vinculin and talin [39, 47, 83, 84]. Ligand coated beads brought in contact with either integrins or cadherins and loaded with force could induce changes in bond strength [85, 86] giving proof for outside-in signaling at adhesion sites. Moreover, it is stated that bond lifetimes in the minimal cadherin/catenin/actin complex increase upon force [87]. Neither integrin nor cadherin adhesion spots are isolated but studies showed that force application upon E-cadherin can even change focal adhesions and further cell contractility [88]. Vice versa, substrate traction forces transmitted via integrins can influence tension measured at cell-cell contacts [89]. Furthermore, recent studies stated that these adhesion sites compete for the recruitment of vinculin, which is a major player for mechanotransduction. In early stages, vinculin is predominantly found at focal adhesions, however, upon monolayer formation, more and more gets recruited to adherens junctions and becomes a mechanosensitive element there [90]. This transition from cell-matrix to cell-cell adhesions might be a crucial switch mechanism in cell mechanics being also important for later discussed collective behavior [91].

All these findings show, that the adhesion sites are the first essential key players in cell mechanosensing and mechanotransduction, especially at the actin coupled adhesion sites. For desmosomes, mechanosensing and -transduction still remains an open question. Due to its connection to the keratin network, internal cell contractility will not directly affect these adhesion sites. However it is consensus, that desmosomes are crucial for maintenance of tissue integrity and morphogenesis [92, 93] especially due to their ability to form hyperadhesive states. Their remodeling seems to be crucial for proper tissue homeostasis [94]. During the last years also evidences were gathered which show that desmosomes might also be able to interfere with mechanotransduction signaling pathways and are not only altered by them (e.g. the hippo pathway [95]). Also 


\section{Introduction}

proteins inside desmosomes fulfill prerequisites to potentially serve as mechanosensors [95]. Moreover, recent studies showed that through interfering with the IF-desmoplakin linkage, cell mechanics, cell-cell forces and cell-substrate forces were altered [96]. In the upcoming years further studies will help to unravel the concrete role of desmosomes actively participating in mechanosensing and mechanotransduction in cell sheets.

Concerning tight junctions, barrier function efficiency can be altered via external stress [97] or changes in cellular contractility [98]. Also tight junction positioning within a tissue is influenced by E-cadherin mediated adhesion and further established contractile forces [59]. In all cases, actin, which is linked to the tight junction complex [33] is a key player but direct implications for tight junctions influencing mechanotransduction are not reported yet. Along the same line, classical AFM studies showed that also apical cell mechanics change only marginally when ZO-1 alone is depleted [99]. Double knockdown of ZO-1 and ZO-2, however, seems to increase apical epithelial tension measured via noncontact acoustic frequency-modulation AFM [100].

All in all, nowadays only adherens junctions and focal adhesions are well identified to actively initiate mechanotransduction at the cells periphery and thereby influence the cells mechanical behavior.

\section{Cytoskeleton}

The overall cell mechanics are determined by the architecture of cytoskeletal components. As seen in section 1.2.2, single filaments of the cytoskeleton have different architecture, filament mechanics and crosslinking which in turn leads to different contributions with respect to the overall cell mechanics. Bundled actin filaments are rather stiff and in the presence of myosin, they exert contractile forces. Also the actin cortex at the cells periphery is a major contributor to cell mechanics [8]. Intermediate filaments are flexible and less resistant to bending [19] but can be stretched at least 2.6 fold [101]. Studies showed that microtubules in contrast bear compressive loads inside cells and buckle under force application [102]. All filaments together build a composite network in the cell resembling a tensegrity structure [103]. Microrheological analysis of this composite network shows the elastic and viscous contributions to overall cell mechanics on different time scales [104], which can be used, e.g., as a marker for the malignancy of cells [2]. The cytoskeleton can rearrange and adapt upon external stimuli. Studies showed that 
after application of cyclic cell stretching, actin stress fibers reorient along the direction of minimal substrate deformation [105] and the amount of stress fibers and coupling to adhesion spots changes [106]. Also during rigidity driven differentiation, diverse actin patterns arise on cells cultured on elastic substrates [107]. Biochemical signaling pathways as the hippo pathway or Rho GTPase signaling are believed to be activated in the sensing mechanism and can induce remodeling of the cytoskeleton [108]. Especially RhoA and Rac1 are of high importance for actin remodelling and mechanotransduction [108]. They can be activated by mechanical stimuli and subsequently drive specific actin remodelling. In this context, Rac is known to promote lamellipodia formation and emergence of branched actin networks while RhoA in combination with the downstream Rho-associated kinase are major players in actomyosin contractility [91].

For microtubules it was shown that the outgrowth in the cells periphery is increased by application of mechanical stress [109]. Moreover, it is known that microtubules are involved in regulation of focal adhesion size [49] and that they are important factors in the front-rear polarization of migrating cells [110]. But a direct role for microtubules in mechanosensing via cell adhesion complexes in cell sheets is not known [108].

Concerning intermediate filaments, strain-stiffening was observed in IF polymer networks [11] being important for cell integrity during force application. Especially the unique filament viscoelastic properties make intermediate filaments an important factor for single cell mechanics upon deformation [112]. With gene engineering it was found that keratinocytes lacking the keratin network exhibit softer cell mechanics when cultured on substrates [72] or when being freely suspended in solution [113]. Also faster adhesion was observed in cells lacking the keratin network [114]. Moreover, in single migrating mesendoderm cells, keratin organization is regulated by tension which is applied on cellcell contacts [115]. Still, the large number of different IFs would allow to vary mechanical properties determined by IFs in different cell types [26]. Along this line, studies suggest that the different keratin expression patterns in keratinocytes during differentiation and wound healing might imply different compliance of the cells [116].

So, studies are arising which show the importance of IFs in single-cell mechanical properties. Also for cell sheet migration, an important role is attributed to intermediate filaments during the last years (see next section) but a general understanding of direct IF-mediated mechanoresponses inside individual cells is still missing [108]. 


\section{Introduction}

\subsection{Collective mechanical behavior of epithelial cells}

Aspects of single cell mechanics especially as response to external signals were discussed above. However, several cells have to work together to contribute to a functioning tissue. This section highlights the resulting cohesiveness and collective behavior emerging in cell cultures and how signals are believed to be transmitted throughout several cells.

Collective cell sheet migration and tissue mechanics are crucial collective phenomena especially during wound repair and regeneration.

\section{Substrate adhesion in collective migration}

One important aspect in collective migration is the underlying substrate with which the cells interact through focal adhesions. Faster migration was observed on stiffer substrates and is explained by the supracellular contractile force transmission [117]. Leader cells in migrating MDCK cell layers directly induce large traction forces to the substrate while follower cells are connected via actomyosin cables being under tension [118]. An important signaling molecule for leader cell formation but also general collective movement was identified called merlin, which is member of the hippo pathway [119]. Moreover, propagating mechanical waves were monitored leading to a coordinated tissue expansion [120]. Through observation it was found that velocity correlation length can reach up to $200 \mu \mathrm{m}$ [69]. Insights into this behavior give studies which found that cell-cell junctions associated with catenins and cadherins are crucial for a coordinated movement on substrates [121, 122]. Along the same line, intercellular forces measured with monolayer stress microscopy seem to work cooperatively between neighboring cells in order to allow collective migration [70].

\section{Actomyosin induced pretension as prerequisite for collective behavior}

In general, the interplay between forces at cell-cell junctions and cell-substrate adhesions seems to be the key to collective migration phenomena. The cells pretensed actomyosin cytoskeleton inbetween these junction complexes transmits and contributes to these forces coupled into and exerted by each cell. So, for migrating cell sheets it is stated that they "can thus be seen as tissues under tension" [123]. This is also nicely demonstrated with the formation of keratinocyte epithelial bridges, which can span non-adhesive areas 
upon migration on micropatterned surface [124].

But also non-migrating epithelial cells show a tissue-level tension. This was found e.g. for MDCK II cells cultured on collagen gels by deep AFM indentation experiments [52] or also for MCF-7 epithelial cells which show an immediate relaxation upon wounding of an area of 10 cells [125]. Upon layer formation after cell seeding, a first steep increase in tissue-level tension coincides with the formation of adherens junctions even though it is noteworthy that traction stresses to the substrate also contribute [52]. These experiments proofed, that actomyosin activity coordinated by junctions is also crucial for building tissue-level tension in non-migrating tissues. Additionally, for longer times, it was found that the monolayer tension stabilizes at a lower steady-state value. During this time, changes in cellular morphology occur which are prerequisite to gain homeostatic cell tensions [52].

\section{General tissue mechanics}

Furthermore, studies with completely freely suspended monolayers, so without any substrate support, were conducted [126]. They showed that after application of low stress at the monolayer, the strain reaches a plateau while for high stress a creep behavior occurs, demonstrating the viscoelastic behavior and a partial fluidization of cell sheets at large strains. Upon monolayer stretching, each cell elongates in the direction of stretch and gets thinner. This demonstrates that single cell mechanics determines also tissue mechanical responses upon stretch. Moreover, cell rearrangements within tissues are an important factor for tissue mechanics. In homeostatic epithelial tissue, cell division, extrusion and intercalation are crucial mechanisms to display a tissue fluidity on longer time scales [127, 128]. Rearrangement of cell-cell contacts is prerequisite to allow tissue remodeling in living tissues.

Simulations allow to tune the contributions of cell elasticity, cortical contractility and cell-cell adhesion on epithelial packing and resulting cell morphologies in monolayered cell sheets [129]. A soft and a hard regime can be classified where either cell-cell adhesion or contractility inside individual cells, dictate cell shape and tissue properties as wound closure [130] or tissue elasticity upon stretching [131]. Only a precise balance of these contributions allows to reproduce experimental results from living cell sheets [129].

This also holds for 3D cell cultures: in 3D keratinocyte cell culture it was found that 


\section{Introduction}

the amount of tension across adherens junctions determines the stability of tight junctions and subsequently proper polarization and function of the epidermal tissue [59]. Especially in 3D systems which are often more comparable to the in vivo tissue, force homeostasis has to be tightly regulated. Local distortions in cell shape and cortical tension are necessary for differentiation and delamination in stratifying epidermis [132]. This is accompanied by an increase in cell-cell adhesion to allow junctional rearrangement and a local fluid-like state so that a single cell delaminates from the basal and enters the suprabasal layer in the epidermis.

So the interplay of cell-cell adhesion and actin-induced contractility are major determinants for collective behavior in epithelial tissue. However as more and more evidence is arising that also the other cytoskeletal filaments and especially IFs contribute to single cell mechanics (section 1.4), also collective behavior can be affected by these filaments.

\section{Contributions from intermediate filaments}

Especially for collective cell sheet migration and cell sheets upon stretch, a major role of intermediate filaments was identified during the last years: It was shown that keratin-free cells exhibit larger invasiveness and increased migration [113, 114]. Also, intermediate filaments are thought to help sensing the direction and strength of applied forces on cell sheets [108]. Studies in migrating Xenopus mesendoderm cell sheets revealed an accumulation of $\mathrm{K} 8 / \mathrm{K} 18$ at spots where force application at cadherins happened [115]. Other studies in migrating cell sheets showed, that intermediate filaments can affect cell migration by regulating traction forces in astrocytes [133] or mesendoderm cells [134]. It is stated that keratin filaments are required to balance stresses in the tissue during collective migration [134].

Also at high strain in freely-suspended monolayers, keratin filaments are a major factor for generating a nonlinear mechanical response through filament alignment and elongation [126]. Another aspect is that specific posttranslational modifications or structural reorganization of intermediate filaments can influence collective behaviors as migration or development [17]. Moreover, the amount of human diseases associated with mutations of IF coding genes shows the importance of IFs for mechanical homeostasis in tissues and organs [135] but the picture is not complete yet. 


\subsection{Micro-wound healing mechanisms of epithelial cells}

Major parts of this section are published in the article Single-cell defects cause a longrange mechanical response in a confluent epithelial cell layer [136]. Text is taken either word-by-word or is closely related.

Wound healing and subsequent closure is a very important physiological process in living organisms [137]. Especially for epithelial cells, which form a tight barrier that prevents molecules to cross the epithelium and provide mechanical stability [138], the integrity and tight regulation of the cell layer and their resulting collective behavior are indispensable [127]. Therefore, proper wound closure also at the $\mu \mathrm{m}$-scale must be ensured to restore tissue homeostasis but parameters like wound size [139, 140] and wound geometry [141] can influence the closing. For closing larger wounds, migration and hence lamellipodia and leader cell formation are important [140, 142,-144]. Contrastingly, for small wounds of the size of one or only few cells, the wound is mostly closed by a multi-step mechanism relying on the action of an actomyosin purse string [145-149]. Depending also on the way of wounding, both mechanisms can be present to different extents [150]. In this context, Trepat and coworkers used traction force microscopy for wounds of the size of $\approx 20$ cells and thereby found a force pattern that can be explained by a two-stage process including both mechanisms [151]. At an early stage, leading actin protrusions from cells adjacent to the wound generate traction forces pointing away from the defect, indicating that wound closure is driven by cell migration. At a later stage, traction forces also point inwards due to action of the actomyosin ring lining the wound and generating tension via focal adhesions to the underlying substrate, which deforms and drags the cell sheet inwards.

For wounding of a single cell in a confluent MDCK II cell layer, as it is performed in this study, it was found that an explicit multistep mechanism takes place: initially the dying cell provides a signal for the adjacent cells by exerting tension on the neighboring cells through a contractile apical F-actin ring [152, 153]. Then, Rho and Rho-kinase localize at the wound margin and a multicellular actomyosin purse string is established at the wound margin [154, 155]. In a next step, myosin light chain kinase is activated and the actomyosin ring starts to contract, while moving from apical to basal [153, 155]. Lastly, F-actin protrusions from the neighbors become visible at the basal plane, which leads 


\section{Introduction}

to the final cell extrusion [153, 155]. Caspase activity and the sphingosine 1-phosphat pathway are essential for the final extrusion process [156, 157].

So the closure process was well studied over the years. But to what extent neighbors are affected and collective behavior is necessary is less well understood and will be in the scope of this thesis.

For keratinocytes, single-cell wound healing is largely unexplored. Large wounds, however, especially in mouse model systems are widely investigated due to their medical relevance. For reepithelialization, keratinocytes have to migrate to wounded areas. During this process, the keratins $\mathrm{K} 6, \mathrm{~K} 16$ and $\mathrm{K} 17$ are upregulated [158]. A possible explanation for this upregulation is that these filaments give the cells pliability on the one hand but sufficient mechanical strength on the other [116]. For homeostatic epidermis, it is postulated that $\mathrm{K} 5 / \mathrm{K} 14$ heterodimers present in cells in the basal layer support more pliability, while $\mathrm{K} 1 / \mathrm{K} 10$ heterodimers present in cells in the suprabasal layers support stronger mechanical resilience [116]. K6, K16 and K17, however, are not expressed in the epidermis under homeostatic conditions in adult humans or mice [74]. In order to be able to migrate, cell-cell and cell-substrate contacts have to be disassembled [158]. It was shown that $\mathrm{PKC} \alpha$ is a major signaling molecule in this process, enabling the cell to switch from the hyperadhesive state to a weaker adhesive calcium-dependent state [46]. Besides migration, also a purse string mechanism, similar to the one in MDCK II cells, was reported already for embryonic epidermal wound closure [74].

In this study, single-cell wounding is applied to keratinocytes to compare the response to MDCK II cells and to analyze the time course of wound closure. A special focus in this section will lie on the presence and absence of the keratin network and how this affects single cell mechanics and collective behavior in the layer upon wounding. 


\section{Materials and methods}

\subsection{Cell culture}

The cell lines used in this work were thankfully cultivated by Anja Herdlitschke, Angela Rübeling and Dr. Tabea Oswald (all University of Göttingen, Germany).

\subsubsection{MDCK II cells}

MDCK II cells (Strain II, Health Protection Agency, Salisbury, United Kingdom) were cultured in Earls minimum essential medium (Lonza, Basel, Switzerland) at $37{ }^{\circ} \mathrm{C}$ and $5 \% \mathrm{CO}_{2} .4 \mathrm{mM} \mathrm{L-glutamine}$ (Biochrom, Berlin, Germany) and $10 \%(\mathrm{v} / \mathrm{v}$ ) fetal calf serum (FCS, BioWest, Nuaillé, France) were added to the medium. Every 2-3 days the cells were subcultured via trypsinization (trypsin/EDTA $0.5 \% / 0.2 \%$, Biochrom, Berlin, Germany). For experiments without sterile conditions, additionally $0.2 \mathrm{mg} / \mathrm{ml}$ penicillin/streptomycin ( $\mathrm{P} / \mathrm{S}, \mathrm{PAA}$, Pasching, Germany) was added to the medium.

\subsubsection{Keratinocytes}

Gene engineering of mice lacking the entire keratin multiprotein family and further isolation of primary keratinocytes from these mice was performed according to Vijayaraj [71] in the group of Prof. Magin (Institute of Biology \& SIKT, University of Leipzig, Germany). Stable cell cultures of wild-type keratinocytes (WT), keratin knock-out cells (Type I, called $\mathrm{Kty}^{-/}$) and cells re-expressing keratins by lentiviral gene transfer (K14) were subcultured and passaged by ourself according to the protocol described by Seltmann in [113]: Cells were grown in FAD medium (Dulbecco modified eagle medium, hams F12, low calcium (50 $\mu \mathrm{M})$, Biochrom, Berlin, Germany, supplements see table 2.1) at $32{ }^{\circ} \mathrm{C}$ and $5 \% \mathrm{CO}_{2}$. Every $3-4$ days they were passaged using trypsin / EDTA 


\section{Materials and methods}

(0.025 \% trypsin / $0.02 \%$ EDTA in PBS, both Biochrom, Berlin, Germany) for few minutes at $37{ }^{\circ} \mathrm{C}$. Cells were seeded in fresh medium at a density of 30,000 cells $/ \mathrm{cm}^{2}$ on collagen coated plastic ware. For collagen coating, collagen I (bovine collagen I, Life Technologies, Carlsbad, USA) was diluted in $0.02 \mathrm{M}$ acetic acid (in ultra pure water, Carl Roth, Karlsruhe, Germany) and incubated for $30 \mathrm{~min}$ at $32{ }^{\circ} \mathrm{C}$, so that a surface coverage of $5 \mathrm{\mu g} / \mathrm{cm}^{2}$ was achieved for every culture ware. Prior to every experiment, one day after seeding, FAD was changed to $\mathrm{FAD}^{+}$medium containing $1.2 \mathrm{mM} \mathrm{CaCl}_{2}$ to establish proper junctions and induce terminal differentiation, which is blocked in normal FAD medium. If not stated otherwise, the experiments were performed $48 \mathrm{~h}$ after calcium switch. For K14 cells, $8 \mu \mathrm{g} / \mathrm{ml}$ puromycin (Sigma-Aldrich, St. Louis, USA) was added to the media at any time.

Table 2.1.: Supplements in FAD medium for keratinocyte cell culture.

\begin{aligned} & \hline Concentration Supplement \\ & \hline $10 \%(\mathrm{v} / \mathrm{v})$ FCS Gold (neutralized chelex treated, \\ & PAA, Pasching, Germany) \\ & $0.18 \mathrm{mM}$ adenine (Sigma-Aldrich, St. Louis, USA) \\ & $0.5 \mu \mathrm{g} / \mathrm{ml}$ hydrocortisone (Sigma-Aldrich, St. Louis, USA) \\ & $2.5 \mu \mathrm{m} / \mathrm{ml}$ insulin (Sigma-Aldrich, St. Louis, USA) \\ & $100 \mathrm{pM}$ cholera toxin (Sigma-Aldrich, St. Louis, USA) \\ & $10 \mathrm{ng} / \mathrm{ml}$ EGF (Invitrogen, Carlsbad, USA) \\ & $1 \mathrm{mM}$ sodium pyruvate (Invitrogen, Carlsbad, USA) \\ & $100 \mu \mathrm{mg} / \mathrm{ml}$ P/S (Invitrogen, Carlsbad, USA) \\ & $2 \mathrm{mM}$ Glutamax (Invitrogen, Carlsbad, USA) \\ & \hline\end{aligned}

\subsubsection{Culture conditions during the experiments}

For experiments, cells were seeded in tissue culture treated petri dishes (ibidi $\mu$-Dish, low, sometimes with grid (Grid-500), Martinsried, Germany) with $3 \mathrm{ml}$ medium. When keratinocytes were used, also the petri dishes were coated with collagen I. In case of no $\mathrm{CO}_{2}$ supply during the experiment, $15 \mathrm{mM}$ HEPES (Biochrom, Berlin, Germany) was added to the culture medium for both cell lines. The heating setup (petri dish heater, JPK Instruments, Berlin, Germany) was set to either $37^{\circ} \mathrm{C}$ (for MDCK II cells) or $32{ }^{\circ} \mathrm{C}$ (for keratinocytes). Medium was changed every 2 hours to compensate the slow $\mathrm{pH}$ 
change observed also in the HEPES buffered medium.

\subsubsection{Transfection of E-Cadherin-GFP cells}

The experimental procedure described in this section was performed together with Angela Rübeling (Institute of Organic and Biomolecular Chemistry, University of Göttingen, Germnay).

In one experiment, MDCK II cells were transfected with E-Cadherin-GFP plasmid (expressed in $\mathrm{DH} 5 \alpha$ cells, Addgene, Cambridge, USA). The bacteria were incubated at $37^{\circ} \mathrm{C}$ overnight on an agar plate before harvesting and culture in LB media (supplements see table 2.2 in deionized water, $\mathrm{pH}$ 7.0). Purification was done via the NucleoBond Xtra Midi EF Kit (Macherey-Nagel, Düren, Germany) following the guidelines of the manufacturer. The DNA content was checked via agarose gel electrophoresis. Plas-

Table 2.2.: Composition of LB media and agar solution used for plasmid amplification.

\begin{tabular}{rl}
\hline Concentration & Supplement \\
\hline $0.01 \mathrm{~g} / \mathrm{ml}$ & $\begin{array}{l}\text { tryptone/peptone ex casein } \\
\text { (Carl Roth, Karlsruhe, Germany) }\end{array}$ \\
$0.005 \mathrm{~g} / \mathrm{ml}$ & yeast extract (Carl Roth, Karlsruhe, Germany) \\
$0.01 \mathrm{~g} / \mathrm{ml}$ & $\mathrm{NaCl}$ (Fluka, Buchs, Switzerland) \\
$100 \mu \mathrm{g} / \mathrm{ml}$ ampicillin (Carl Roth, Karlsruhe, Germany) \\
$\mathrm{added}$ only for agar plate: \\
$15 \mathrm{~g} / \mathrm{l}$ agar (Carl Roth, Karlsruhe, Germany) \\
\hline
\end{tabular}

mid insertion into MDCK II cells was done with a transfection system consisting of Gene Pulser Xcell (Bio-Rad Laboratories, Munich, Germany) including a ShockPod cuvette chamber (Bio-Rad Laboratories, Munich, Germany) and a CE module (Bio-Rad Laboratories, Munich, Germany). $75 \%$ confluent grown cells were trypsinated (trypsin/EDTA $0.5 \% / 0.2 \%$; Biochrom, Berlin, Germany) and centrifuged $\left(4{ }^{\circ} \mathrm{C}, 1200 \mathrm{U} / \mathrm{min}, 3 \mathrm{~min}\right)$. The pellet was resuspended in $1 \mathrm{ml}$ ice-cold OptiMEM (Life Technologies, Carlsbad, USA) and diluted to $2.5 \times 10^{6} \mathrm{cells} / \mathrm{ml}$. $400 \mu \mathrm{l}$ cell suspension was filled in an ice-cold cuvette ( $4 \mathrm{~mm}$ gap width) and $25 \mu \mathrm{g}$ of plasmid was added. Electroporation was done with $250 \mathrm{~V}$ and $950 \mu \mathrm{F}$ as electrical parameters. The suspension was quickly added to $500 \mu \mathrm{l}$ culture medium with double amount of FCS $(20 \%(\mathrm{v} / \mathrm{v})$, BioWest, Nuaillé, 


\section{Materials and methods}

France) and seeded in a petri dish. One day after electroporation G418 (800 $\mu \mathrm{g} / \mathrm{ml}$, Carl Roth, Karlsruhe, Germany) was added to kill untransfected cells.

\subsection{Wounding strategies for a confluent cell layer}

Several types of wounding strategies were employed during this study which will be described in the following. In general, wounding on the cell scale is not defined properly. Here, it will be employed as a failure or the general absence of cell neighbors in an intact surrounding cell layer.

\subsubsection{Induction of large wounds}

Holes of at least several cell widths are classified as large wounds. Three different strategies were employed to create these.

\section{Use of a culture insert}

A classical way to induce large wounds are culture inserts (figure 2.1 A). Via 2 well silicone inserts (ibidi, Martinsried, Germany) a defined cell free gap of $500 \mu \mathrm{m}$ width can be created when detaching the silicone. 6,000 MDCK II cells in $70 \mu$ medium were seeded in each chamber and 175,000 cells in $1 \mathrm{ml}$ in the outer part. Cells were cultured for 2 days before the experiment. For the experiment, the silicone insert was removed with a sterile tweezer and the medium was changed to HEPES and P/S containing medium.

\section{Performance of a scratch assay}

Scratch assays are easy and well-developed assays for mimicking wound healing [159] (figure 2.1B). Via a steril pipette tip (200 $\mu$, Eppendorf, Hamburg, Germany), a scratch was manually induced in an already confluent grown layer.

\section{Intrinsic wounds in not fully confluent cell layer}

Furthermore, intrinsic holes in a cell layer were investigated (figure 2.1 C). Shortly before complete confluency, small holes are still present in the everywhere else homogeneous 
cell layer. In our case, this was achieved with a seeding density of 125,000 cells/ibidi dish and culture for 2 days.

A

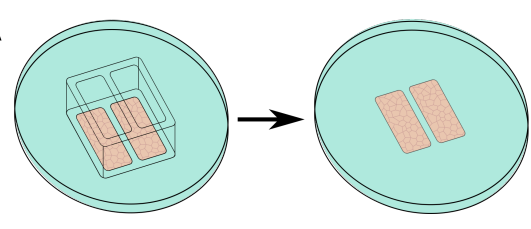

B

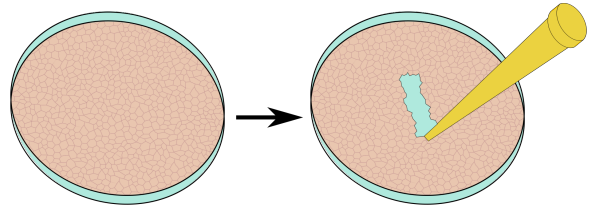

C

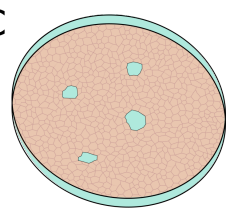

Figure 2.1.: Three methods were used to create cell layers with large wounds. (A) Either a culture insert was used or (B) a classical scratch assay with a pipette was performed. Lastly, (C) not fully confluent grown cells were used for getting cells without proper neighbor connections.

\subsubsection{Induction of single-cell defects}

Additionally, single-cell defects were created in a homogeneous confluent cell layer (figure 2.2.). If several defects per dish were induced, separation widths of minimal $200 \mu \mathrm{m}$ were in between the defects to avoid cross-talk of effects.

\section{Micropipette action}

One technique to induce a single cell defect is mechanical wounding of a single cell. Here, a micromanipulator setup (Femtojet and InjectMan NI2, Eppendorf, Hamburg, Germany) was used to mechanically scratch across a cell via $x-y$ movements of a glass capillary (Femtotip, Eppendorf, Hamburg, Germany) (figure 2.2 A). A change in phase contrast indicates successful wounding.

\section{Laser ablation}

The experimental procedure described in this paragraph was performed together with D. Kong (Institute for Developmental Biochemistry, Medical School, University of Göttingen).

Laser ablation is commonly used on the single cell level but also on the subcellular level to sever cell compartments very precisely [160]. Here, it was used to induce a single-cell wound non-mechanically (figure 2.2 B) as a control to the above mentioned method. The microscope setup did not allow heating for the time of wounding. The laser ablation setup (DSPL-355/14, 355 nm, 70 mJ/pulse; Rapp Optoelectronic, Wedel, Germany) 


\section{Materials and methods}

was adjusted so that no cavitation bubble occured destroying the neighbors. This was achieved with the click and fire mode with 40 pulses within $200 \mathrm{~ms}$ and a laser power of 2 - 3\%. A 40x oil objective (NA 1.3, EC Plan-NEOFLUAR; Carl Zeiss, Oberkochen, Germany) was used during the ablation. A change in contrast shortly after the laser pulses indicates success in cell ablation.
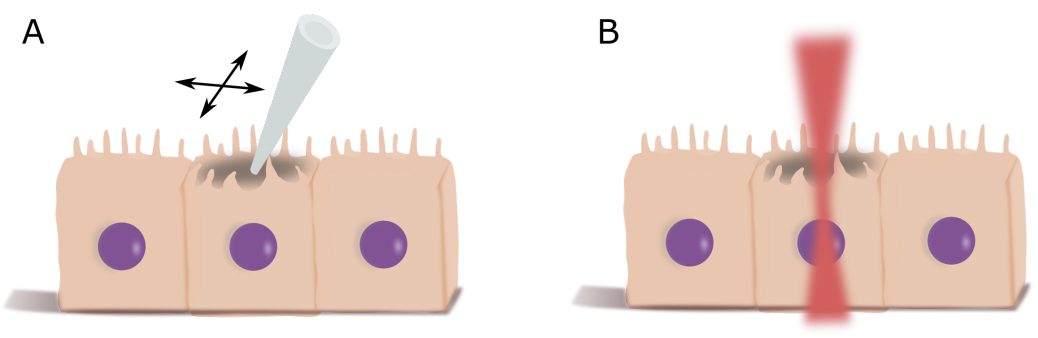

Figure 2.2.: Two methods were used to create single-cell defects. (A) Micropipette action mechanically destroyed a cell while (B) laser ablation was a contact-less method to create a single-cell wound.

\subsection{Light microscopy techniques}

During this thesis, phase contrast, conventional fluorescence and confocal laser scanning microscopy were used as light microscopy imaging techniques. As these techniques are nowadays standard microscopy techniques for a lot of research topics, the physical principles will not be explained here. For further information, the reader is referred to prominent text books such as [161].

\subsubsection{Cell labeling}

Cells were labeled according to a standard protocol:

At the desired time point, cells were washed with $\mathrm{PBS}^{-/-}$before fixation with paraformaldehyde (Fluka, Buchs, Switzerland, $4 \%$ in $\mathrm{PBS}^{-/}$) for $20 \mathrm{~min}$ at room temperature. Permeabilization of the membrane and blocking of unspecific binding was achieved by adding blocking buffer (5\% (w/v) bovine serum albumin (BSA, IgG free, Carl Roth, Karlsruhe, Germany) and $0.3 \%$ (w/v) Triton-X 100 (Sigma-Aldrich, St. Louis, USA) in $\mathrm{PBS}^{-/-}$) for $30 \mathrm{~min}$ at room temperature.

Either two step labeling with a primary antibody targeting the specific protein and a 
fluorescently labeled secondary antibody or direct one-step labeling were applied. All dyes and antibodies were diluted in dilution buffer $(1 \%(\mathrm{w} / \mathrm{v})$ BSA and $0.3 \%(\mathrm{w} / \mathrm{v})$ Triton-X 100 in PBS) to the desired concentration and incubated as seen in table 2.3. Between labeling steps, cells were rinsed three times with $\mathrm{PBS}^{-/-}$for $5 \mathrm{~min}$ and placed on a shaker.

Table 2.3.: The antibody targeted structures with incubation time and concentration of the used antibodies.

\begin{tabular}{|c|c|c|c|}
\hline Target & Reagent & Conc. & $\begin{array}{l}\text { Incubation } \\
\text { time }\end{array}$ \\
\hline E-cadherin & $\begin{array}{l}\text { mouse anti-E-cadherin IgG2a } \\
\text { (BD Biosciences, Heidelberg, Germany) }\end{array}$ & $5 \mu \mathrm{g} / \mathrm{ml}$ & $1 \mathrm{~h}$ \\
\hline ezrin & $\begin{array}{l}\text { mouse anti-ezrin IgG1 } \\
\text { (BD Biosciences, Heidelberg, Germany) }\end{array}$ & $2.5 \mu \mathrm{g} / \mathrm{ml}$ & $1 \mathrm{~h}$ \\
\hline F-actin & $\begin{array}{l}\text { Alexa Fluor } 488 \text { or Alexa Fluor } 546 \\
\text { conjugated Phalloidin } \\
\text { (Life Technologies, Carlsbad, USA) }\end{array}$ & $165 \mathrm{nM}$ & $45 \mathrm{~min}$ \\
\hline keratin & $\begin{array}{l}\text { anti-cytokeratin } 14, \text { mouse monoclonal } \\
\text { (Abcam, Cambridge, UK) }\end{array}$ & $1 \mu \mathrm{g} / \mathrm{ml}$ & $1 \mathrm{~h}$ \\
\hline $\begin{array}{l}\text { podocalyxin } \\
\text { (GP 135) }\end{array}$ & $\begin{array}{l}\text { monoclonal MlgG1 } \\
\text { (DSHB Hybridoma Product 3F2/D8) }{ }^{1}\end{array}$ & $5 \mu \mathrm{g} / \mathrm{ml}$ & $1 \mathrm{~h}$ \\
\hline DNA & $\begin{array}{l}\text { 4',6-diamidino-2-phenylindole (DAPI) } \\
\text { (Life Technologies, Carlsbad, USA) }\end{array}$ & $50 \mathrm{ng} / \mathrm{ml}$ & $15 \mathrm{~min}$ \\
\hline ZO-1 & $\begin{array}{l}\text { mouse anti-ZO-1 } \\
\text { (Thermo Fisher Scientific, Waltham, USA) }\end{array}$ & $5 \mu \mathrm{g} / \mathrm{ml}$ & $1 \mathrm{~h}$ \\
\hline $\begin{array}{l}\text { secondary } \\
\text { antibody }\end{array}$ & $\begin{array}{l}\text { Alexa Fluor } 488 \text { or Alexa Fluor } 546 \text { lgG goat- } \\
\text { anti-mouse } \\
\text { (Thermo Fisher Scientific, Waltham, USA) }\end{array}$ & $5 \mu \mathrm{g} / \mathrm{ml}$ & $1 \mathrm{~h}$ \\
\hline
\end{tabular}

\subsubsection{Chemicals used during cell experiments}

To analyze the single-cell wound closure, one inhibitor and a calcium indicator were used:

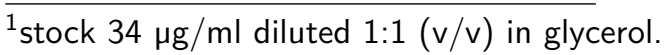




\section{Materials and methods}

\section{Rho-kinase inhibitor Y27632}

The rho-kinase inhibitor Y27632 (dihydrochloride monohydrate, Sigma-Aldrich, St. Louis, USA) was added in a concentration of $10 \mu \mathrm{M} 1 \mathrm{~h}$ prior to the wounding experiment. Also during wound closure it was still present in the medium.

\section{Calcium indicator}

The calcium indicator Fluo-4 calcium imaging kit (Thermo Fihser Scientific, Waltham, USA) was used according to the protocol of the supplier: The probenecid was diluted in

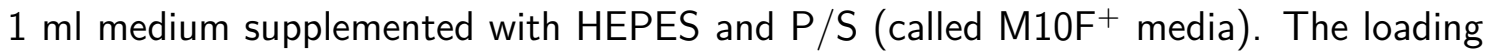
solution consisted of $1 \%(\mathrm{v} / \mathrm{v})$ power load concentrate, $0.1 \%(\mathrm{v} / \mathrm{v})$ Fluo-4-AM solution

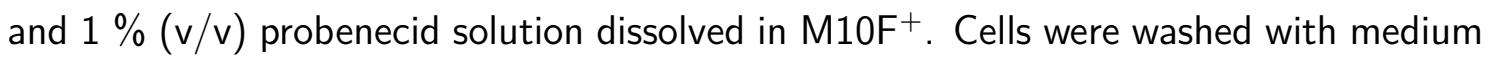
before incubation with loading solution at $37{ }^{\circ} \mathrm{C}$ and room temperature for $20 \mathrm{~min}$. Cells were washed once more with medium before being used for the experiment. During the experiment, $1 \%(\mathrm{v} / \mathrm{v})$ neuro backdrop background suppressor solution (also component of the kit) was added to the medium.

\subsubsection{Video and image acquisition}

Fluorescence and phase contrast microscopy were performed with an Olympus IX 81 microscope (Olympus, Tokyo, Japan) equipped with a 10x objective (UPLFLN 10X2PH, $N A=0.3$, Olympus, Tokyo, Japan) and a 40x objective (LUCPLFKN 40XPH, NA=0.6, Olympus, Tokyo, Japan) and an XM10 camera (Olympus, Tokyo, Japan). For fluorescence illumination an EXFO X-cite 120 PC lamp (Excelitas Technologies Corp., Waltham, USA) and for bright field illumination a $12 \mathrm{~V} / 100 \mathrm{~W}$ halogen lamp (U LH 100, Olympus, Tokyo, Japan) were used. Image acquisition was done with the cellsens dimension software (Olympus, Tokyo, Japan).

For bright field imaging with the 40x objective, the exposure time was adjusted to $50 \mathrm{~ms}$ and the light intensity to $5 \mathrm{~V}$. For fluorescence imaging, 200 - $400 \mathrm{~ms}$ exposure time and $100 \%$ light intensity were used. When using the $10 x$ objective, the exposure time was reduced accordingly. For capturing videos, the experiment manager embedded in the software was programmed in a way that the sample was only exposed to light during image acquisition. For characterizing the closure process, video acquisition was started 
immediately after wounding with a frame rate of 1 frame per minute. For recording the wounding process, the live mode was used capturing images roughly every 80 milliseconds.

Confocal laser scanning microscopy (CLSM) was done with the FluoView1200 system (Olympus, Tokyo, Japan) equipped with a 100x oil-immersion objective (UPLFLN $100 \mathrm{XO} 2 \mathrm{PH}$, numerical aperture $(\mathrm{NA})=1.3$; Olympus, Tokyo, Japan). Diode lasers of 405, 488 and $561 \mathrm{~nm}$ wavelength were used for excitation. A scan rate of $20 \mu \mathrm{s} / \mathrm{pixel}$ was chosen for images with the size of 1024 pixel x 1024 pixel $(127 \mu \mathrm{m} \times 127 \mu \mathrm{m})$. The laser intensities were set to $2 \%$ and the detector sensitivity (HV-value) was adjusted to 500. Each scan line was scanned twice using the kalman filter option. The pinhole size was set to $185 \mu \mathrm{m}$ for slices with a height of $0.5 \mu \mathrm{m}$.

Depending on the brightness in the resulting images, slight adjustments of the imaging parameters were performed deviating from the above mentioned standard imaging parameters.

\subsubsection{Video and image processing}

Videos and images were mostly processed with ImageJ [162], the exception were images acquired with high magnification at the CLSM, which were processed with the Imaris software (Olympus, Tokyo, Japan). Only linear brightness and contrast adjustments were made during processing.

Geometrical parameters of the wounded cells and their neighbors were measured by manually selecting cell boundaries from fluorescence or phase contrast images.

\subsubsection{Cell labeling and image acquisition protocol for keratinocyte experiments}

The experimental protocol for samples prepared and imaged by Fanny Büchau (Institute for Biology \& SIKT, University of Leipzig, Germany) which are presented in section 3.5. deviated from the above mentioned protocol and read as follows:

For staining tight junction proteins, fixation was done by incubation for $30 \mathrm{~min}$ with icecold ethanol and $3 \mathrm{~min}$ incubation with acetone at room temperature before finally three washing steps were performed with tris-buffered saline (TBS). For staining $\alpha$-tubulin, 


\section{Materials and methods}

cells were fixed for $5 \mathrm{~min}$ at $-20{ }^{\circ} \mathrm{C}$ in methanol. For all other proteins, cells were incubated for $10 \mathrm{~min}$ at $4{ }^{\circ} \mathrm{C}$ in $4 \%$ formalin solution (diluted in PBS), then washed 3 times with $\mathrm{NH}_{4} \mathrm{Cl}$ and once with TBS.

Permeabilization was done via 5 min incubation with $0.1 \%$ Triton/PBS. All antibodies were diluted in a solution of $1 \%$ BSA in TBS according to table 2.4 and incubated for $1 \mathrm{~h}$. Between primary and secondary antibody incubation, samples were washed 3 times in TBS for 5 min. F-actin staining with Phalloidin-Alexa488 (diluted 1:40, PromoCell, Heidelberg, Germany) was performed simultaneously with the incubation of the secondary antibody. Finally the slides were washed 3 times in TBS for 5 min each, briefly rinsed in aqua bidest and mounted with Prolong Gold (Invitrogen, Carlsbad, USA). Images were collected using a Axiolmager (Carl Zeiss, Oberkochen, Germany) equipped with Apotome.2 (Carl Zeiss, Oberkochen, Germany) using a 63x/ 1.4 NA oil immersion objective and an AxioCam MRm (Carl Zeiss, Oberkochen, Germany). Image analysis and processing were performed using Zen Software 2012 (Carl Zeiss, Oberkochen, Germany) and Photoshop CS6 (Adobe Systems, San José, USA).

Table 2.4.: The antibodies inculding dilution used for keratinocyte experiments presented in section 3.5

\begin{tabular}{llll}
\hline Antibody & Supplier & Dilution & Host \\
\hline primary antibodies: & & & \\
occludin (OC-3F10) & Thermo Fisher Scientific, Waltham, USA & $1: 250$ & mouse \\
ZO-1 & Thermo Fisher Scientific, Waltham, USA & $1: 200$ & rabbit \\
claudin1 & self-made by AG Niessen (Cologne) & $1: 400$ & rabbit \\
claudin4 & Thermo Fisher Scientific, Waltham, USA & $1: 400$ & rabbit \\
E-cadherin (ECCD-2) & Invitrogen, Carlsbad, USA & $1: 1000$ & rat \\
p120-catenin (15D2) & Santa Cruz, Dallas, USA & $1: 100$ & mouse \\
$\begin{array}{l}\text {-tubulin } \\
\text { myosin-light chain 2 }\end{array}$ & Abcam, Cambridge, UK & $1: 1000$ & rabbit \\
Phospho-myosin-light & Cell Signaling Technology, Danvers, USA & $1: 100$ & rabbit \\
chain (Thr18/Ser19) & & $1: 100$ & rabbit \\
tubulin & Sigma Aldrich, St. Louis, USA & $1: 500$ & mouse \\
secondary antibodies: & & & \\
anti-mouse-, anti-rabbit-, & Dianova, Hamburg, Germany & $1: 800$ & donkey \\
anti-rat-Alexa488, Cy3 & & & \\
\hline
\end{tabular}




\subsection{Patterning of confluent cell layers}

Defined collagen patterns were created on glass bottom dishes via the microscale plasmainitiated protein patterning ( $\mu$ PIPP, figure 2.3 A) described by Segerer et al. [163]. The protocol was established in close cooperation with S. Nehls [164]. Poly-(dimethylsiloxane) (PDMS) was mixed containing 10 times more base than curing agent (Sylgard Elastomer Kit, Dow Corning, Midland, USA). The mixture was degassed for $15 \mathrm{~min}$ and poured onto a silicon wafer with the desired pattern. Hardening took place at $50{ }^{\circ} \mathrm{C}$ within $4 \mathrm{~h}$. The PDMS stamp was pressed on the glass bottom dish and placed in oxygen plasma for $1 \mathrm{~min}$. $50 \mu \mathrm{l}$ of $0.5 \mathrm{mg} / \mathrm{ml}$ of PLL-PEG-TRITC (poly(L-lysine)(20 kDa)-grafted [3.5] with poly(ethylene glycol) $(2 \mathrm{kDa})$ and labeled with tetramethylrhodamine; SuSoS, Dübendorf, Suisse) was pipetted in the dish close to the stamp and left to dry for 1 h. By capillary forces the solution is sucked between the PDMS giving rise to a regular pattern (figure $2.3 \mathrm{~B}$ ). The stamp was removed and after washing with $\mathrm{PBS}^{-/-}$, collagen $\left(20 \mu \mathrm{g} / \mathrm{ml}\right.$ in $\left.\mathrm{PBS}^{-/-}\right)$was added for $1 \mathrm{~h}$ before finally washing with $\mathrm{PBS}^{-/}$.

Visualization of collagen was done via antibody incubation. As primary monoclonal anti-collagen type I antibody (mouse, Sigma-Aldrich, St. Louis, USA) was used in a concentration of $9.4 \mu \mathrm{g} / \mathrm{ml}$ diluted in $\mathrm{PBS}^{-/}$and Alexa Fluor 488 (see table 2.3) as the secondary antibody. For experiments, cells were seeded on the patterns in different densities and fixed for staining as described earlier in this thesis.

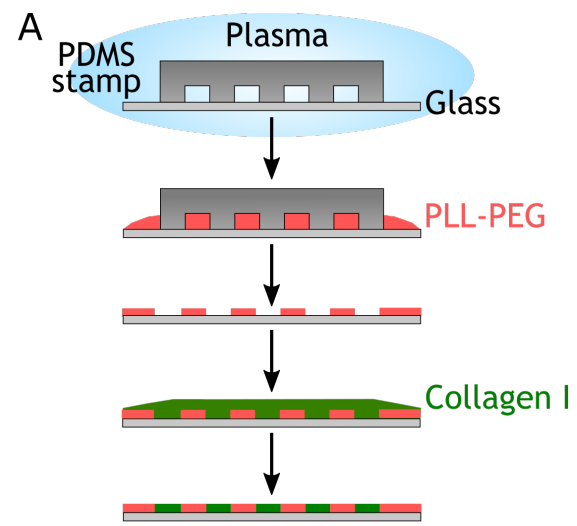

B

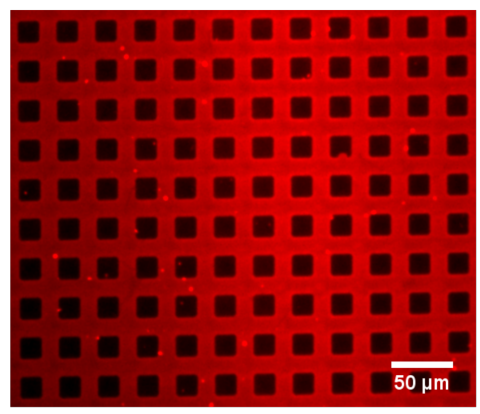

Figure 2.3.: Micro-patterning on a glass according to the protocol by Segerer et al. [163]. (A) PDMS stamp and the glass are activated in oxygen plasma. PLL-PEG is first used to block sites for cell adhesion while collagen binds to free spaces after stamp removal and enables cell adhesion there. (B) Thereby, a regular pattern (PLL-PEG shown in red) can be transferred to the glass. 


\section{Materials and methods}

\subsection{Atomic force microscopy}

The atomic force microscope (AFM) was invented in 1986 by Binnig and Quate as an improvement of the scanning tunnel microscope [165]. Since then it has become a very versatile tool widely used in science. The basic idea is that a nanometer-sized tip comes in contact with a surface while the normal forces and the position of the tip are recorded simultaneously. This allows to image surface topographies on the one hand but also allows to investigate their mechanical properties on the other hand. Both measurement modes were used and will be explained here. As this method plays a central role in this thesis and is not a standard technique in most laboratories, also the fundamentals will be explained in more detail here.

\subsubsection{Basic setup of an AFM}

The basic idea of an AFM is shown in figure 2.4 A. The central piece of an AFM, the cantilever, is brought in contact with the sample. Depending on the interaction with the sample, the tip will feel an attractive or repulsive force and the cantilever will bend accordingly. Via a laser directed onto the cantilever, the deflection can be correlated to a voltage change on a quadrant photo diode. By recording a force curve on a rigid sample, the proportionality factor between the voltage signal and the cantilever bending during contact can be calculated. Also the spring constant $\kappa$ can be derived with a calibration routine which is based on the thermal fluctuations of the cantilever [166]. Both parameters are necessary to convert the photo diode signal with the help of Hookes law to the actual force. In the scanning mode, a certain cantilever bending serves as a set point which is used to adapt the cantilever height accordingly during $x y-$ scanning. Therefore, the real surface topography can be converted into a digital image. In this thesis, only the contact mode is used. Here, the cantilever tip is dragged along the surface and is in permanent contact to the sample while other imaging modes use cantilever oscillations to probe the topography.

Force curves were performed so that the cantilever is lowered in $z$ direction onto the sample. At a certain indentation force set point, the movement is reversed and the cantilever is retracted again. Both, the indentation part after the tip is brought in contact with the sample (figure $2.4 \mathrm{~A}^{\prime}$ ), but also the retraction part where interactions 
still bend the cantilever downwards (figure $2.4 \mathrm{~A}$ ") bears information on the mechanical characteristics of the sample:

If the sample is as soft as the cantilever, both will be deflected during the indentation. In this case the contact region is non-linear, which is due to the change in the contact area of the tip and the sample (figure $2.4 \mathrm{~B}$, black curve). The force-indentation-relationship is commonly described with the Hertz model (equation 2.1, [167]) or Sneddon model (equation 2.2. [168]) depending on the tip geometry. In both cases, one fit parameter is used to calculate the Young's modulus $E$ of the sample.

$$
\begin{aligned}
& F=\frac{4 E \sqrt{r}}{3\left(1-\nu^{2}\right)} d^{\frac{3}{2}} \\
& F=\frac{2 E \tan \alpha}{\pi\left(1-\nu^{2}\right)} d^{2}
\end{aligned}
$$

Here, $\nu$ is the Poisson's ratio ( $\nu=0.5$ for incompressible samples) and $\mathrm{d}$ the indentation depth. For a sphere, the radius $r$ has to be taken into account while for a cone the half-opening angle $\alpha$ as indenter shape characteristic is considered.

Derivation of this model needs to assume a homogeneous and isotropic material. However, this is not the case for biological samples. An alternative to analyze force curves, the tension model, will be explained in the next section.

The cantilever retraction forces shine light on adhesive properties of the sample. This can be due to ligand-receptor interactions (when the tip is specifically coated with a protein) or unspecific binding. Here, unspecific binding is used to pull membrane tethers from the cells surface (figure $2.4 \mathrm{~A}$ " and 2.4 B light grey). However, with e.g. cells glued to the cantilever, whole cell adhesion properties on specific protein coated surfaces can be measured as well.

After a general introduction of atomic force microscopy, now the applied tension model and the experimental steps will be in focus.

\subsubsection{Tension model}

Force curves were analyzed according to the tension model which was previously introduced in the context of cells [8, 169, 170]. In this model, the cell is treated as a prestressed surface around a liquid body which was already hinted at in earlier sec- 


\section{Materials and methods}

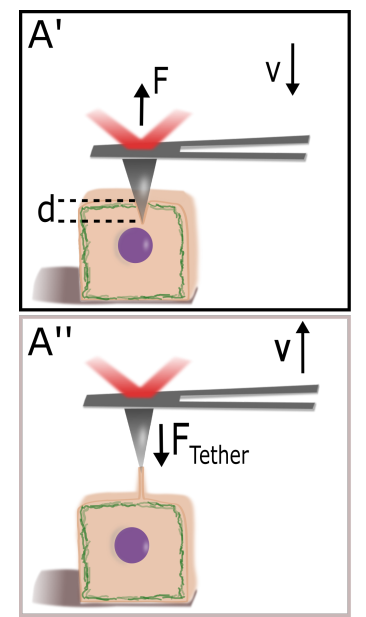

$B$

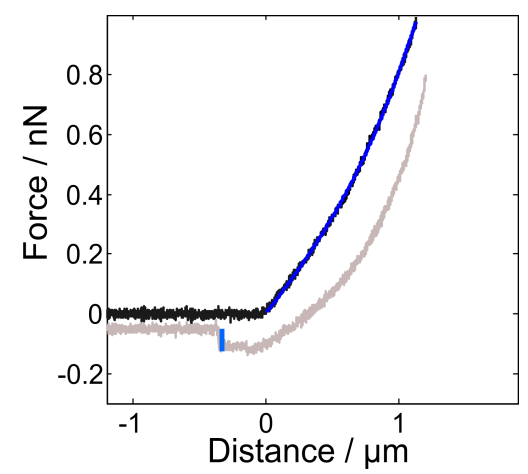

Figure 2.4.: Probing the cells' mechanical properties with an atomic force microscope. (A') A soft cantilever is lowered until an indentation set point force at maximal indentation depth $d$ is reached. To measure the force $F$, a laser directed onto the cantilever, is used. (A") During retraction, adhesive forces due to e.g. membrane tube pulling can be quantified. (B) Fit (blue) of a force indentation curve (black) allows to quantify the cells' mechanics. Rupture forces in the retraction curve (light grey) are visible as sudden force jumps.

tions. For cells aspirated in a micropipette this assumption is obvious and the surface tension origins from a shell build up by the cytoskeletal cortex and the surrounding membrane. For adherent epithelial cells, this model can also be applied. Here, the apical part of the cell, which is exclusively probed by the tip, is mostly dominated by the cytoskeleton-membrane interplay. To account for the origin of the isotropic tension $T$ resisting indentation, the cells pretension $\mathrm{T}_{0}$ and the area dilatation upon indentation has to be taken into account (equation 2.3).

$$
\mathrm{T}=\mathrm{T}_{0}+\mathrm{K}_{\mathrm{A}} \frac{\Delta \mathrm{A}}{\mathrm{A}_{0}}
$$

$\mathrm{K}_{\mathrm{A}}$ is the area compressibility modulus and $\Delta \mathrm{A}$ is the area change compared to the initial area $A_{0}$. For large area expansion, membrane from reservoirs such as protrusions or caveolae has to be recruited as the membrane would otherwise rupture under expansion of more than $\approx 3 \%$ [171].

In low indentation $T_{0}$ is predominant which composes the membrane tension $T_{m}$ and tension contributions from the contractile actomyosin cortex $T_{\text {act }}$ (equation 2.4). To distinguish major contributions, the membrane tension alone can be estimated from 
tether pulling experiments. Here, hollow membrane tubes form which are devoid of an intact cytokeleton. The tether force $F_{t}$ measured during tether rupture is related to the membrane tension as indicated in equation 2.5] [11] and combines contributions from the membranes' in-plane tension and contributions from the attachment of the cytoskeleton to the membrane [8].

$$
\begin{aligned}
\mathrm{T}_{0} & =\mathrm{T}_{\mathrm{m}}+\mathrm{T}_{\mathrm{act}} \\
\mathrm{T}_{\mathrm{m}} & =\frac{\mathrm{F}_{\mathrm{t}}^{2}}{8 \kappa \pi^{2}}
\end{aligned}
$$

$\kappa$ indicates the bending stiffness of the membrane and is on average $20 \mathrm{k}_{\mathrm{B}} T$ [172]. During indentation, if conformal contact with a conical indenter and an originally flat surface is assumed, the following derivation of the tension is valid (figure 2.5):

$$
\mathrm{F}=\int_{0}^{2 \pi \mathrm{a}} \mathrm{T} \sin \Theta \mathrm{ds}=2 \pi \mathrm{Td} \cos \Theta
$$

Here, $a$ is the contact radius at surface level, $\Theta$ the base angle, $s$ the edge length of the cone and $d$ the indentation depth. The flat surface is assumed to have tension contributions as indicated above for cells (equation 2.3). With the new surface area $\mathrm{A}_{\mathrm{n}}=\pi \mathrm{as}+\left(\mathrm{A}_{0}-\pi \mathrm{a}^{2}\right)$, the total change in area is:

$$
\Delta A=A_{n}-A_{0}=\frac{\pi d^{2}}{\sin ^{2} \Theta}\left(\cos \Theta-\cos ^{2} \Theta\right)
$$

Insertion into equation 2.6 and integration gives the force

$$
F=2 \pi T_{0} \cos \Theta \cdot d+\frac{2 \pi^{2}}{\tan ^{2} \Theta A_{0}} K_{A}(1-\cos \Theta) \cdot d^{3} .
$$

This shows, that force curves obtained with a conical indenter can be fitted with a polynomial consisting of a linear and a cubic term. The linear fit coefficient is dominated by the pretension $T_{0}$ and the cubic coefficient by the area compressibility modulus $K_{A}$. 


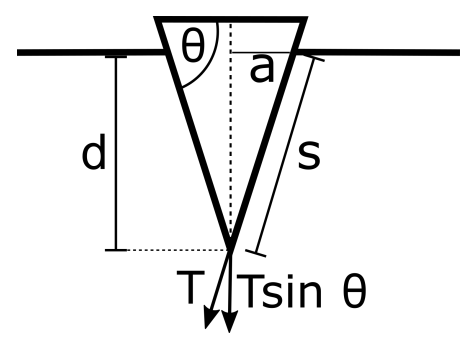

Figure 2.5.: A cone indenting a flat surface. By assuming conformal contact the tension contributions can be calculated from geometrical considerations.

\subsubsection{Force curve recording and processing}

Force curves were recorded with a NanoWizard II/IV AFM (JPK Instruments, Berlin, Germany). Calibration to measure the sensitivity and the spring constant was done by applying the thermal noise method [166] for every used cantilever. These were $C$ levers from MLCT-Cantilevers (Bruker AFM Probes, Camarillo, USA) with a nominal spring constant of $10 \mathrm{pN} / \mathrm{nm}$. For tether pulling, the cantilevers were first cleaned with isopropanol and demineralized water before coating. Coating was done by incubation with $2.5 \mathrm{mg} / \mathrm{ml}$ concanavalin A-FITC conjugate (Sigma-Aldrich, St. Louis, USA) in PBS for 90 minutes at room temperature.

For force indentation experiments, force maps with a resolution of 2 or $4 \mu \mathrm{m} /$ pixel were recorded. Individual force curves were performed with a tip velocity of $3 \mu \mathrm{m} / \mathrm{s}$ and a set point of $1 \mathrm{nN}$. A dwell of $0.5 \mathrm{~s}$ at maximal indentation depth in constant height mode increased the likelihood of tether pulling. By visual inspection, force curves were baselinecorrected and a contact point was defined, both with help of a home-written MATLAB (The MathWorks, Natick, USA) script kindly provided by Dr. Ingo Mey (Institute for Organic and Biomolecular Chemistry, University of Göttingen, Germany). The contact regime was fitted with the polynomial

$$
F(d)=\tilde{T}_{0} \cdot d+\tilde{K}_{A} \cdot d^{3}
$$

in accordance with the above described tension model. If the contact point could not be unequivocally assigned or a drop in the force signal was found in the indentation part (indicating a breakthrough of the membrane) the force indentation curves were excluded from analysis. In the retraction part, force jumps after a force plateau were measured to obtain tether forces $F_{t}$. 
Statistical analysis was done with the Wilcoxon rank sum test implemented in the MATLAB-software with $* * *$ indicating $\mathrm{p}<0.001, * *$ indicating $0.001<\mathrm{p}<0.01$ and $*$ indicating $0.01<\mathrm{p}<0.05$.

\subsubsection{Topography scans}

For topography scans cells were chemically fixed (20 min incubation at room temperature with $4 \%$ paraformaldehyde (Fluka, Buchs, Switzerland) in $\mathrm{PBS}^{-/-}$and kept in $\mathrm{PBS}^{-/-}$ after washing. Topography scans were performed with a NanoWizard IV AFM (JPK Instruments, Berlin, Germany) and an MLCT-Cantilever (Bruker AFM Probes, Camarillo, USA) in contact mode. After equilibration at room temperature, the lowest possible set point was chosen so that the topography could still be captured. Gains were adjusted so that no ringing (oscillations in signal due to over-regulation) was observed. A scan rate of $1 \mathrm{~Hz}$ or $0.5 \mathrm{~Hz}$ was chosen for these comparably soft samples.

\subsection{Electric cell-substrate impedance sensing}

Electric cell-substrate impedance sensing (ECIS) was invented in the early nineties by Lo, Keese and Giaever to monitor the motion of confluent tissue cells [173, 174]. The idea is that electrodes change their electric properties when cells are cultured on them (figure 2.6 A). By applying an AC current between the working and counter electrode, the frequency dependent impedance can be monitored over time allowing to judge the cells' motion and barrier properties. In an electrode chamber filled only with cell medium, the low frequency regime is dominated by the electrical properties of the naked electrode having a powerlaw characteristic of a constant phase element (figure $2.6 \mathrm{~B}$ ). At the high frequency regime the bulk resistance of the medium can be estimated. After seeding cells on the electrodes, an impedance increase can be monitored which is mainly influenced by the permittivity of the cell layer, the cell-substrate distance and the membrane capacitance [175].

For keratinocyte experiments, 8W1E eletrode arrays (8W1E PET ECIS culture ware, Ibidi, Munich, Germany) and the Z $\Theta$ set-up (Applied Biophysics, Troy, USA) were used with the actual samples placed in an incubator at standard culture conditions. The electrodes were coated with collagen $A$ according to the coating protocol described in 

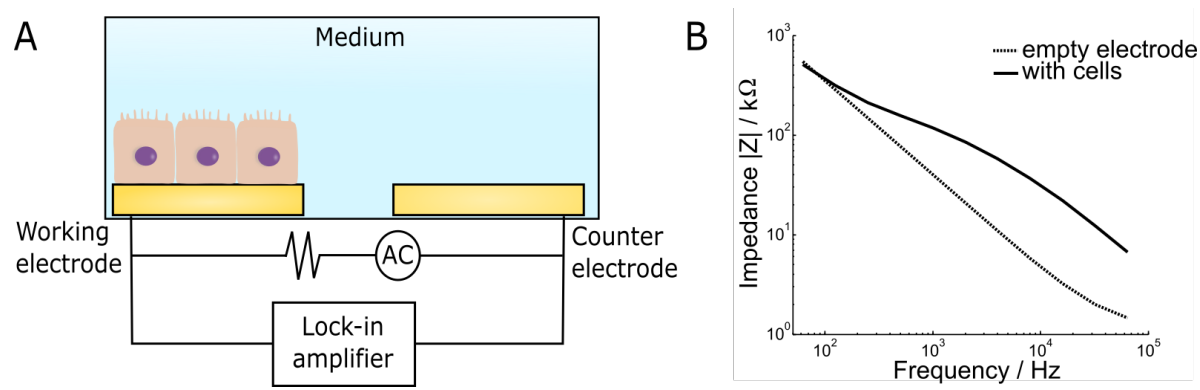

Figure 2.6.: The principle of electric cell-substrate impedance sensing. (A) Cells are seeded on gold electrodes where an alternating current is applied. (B) The impedance spectrum of an empty electrode (dashed) is mainly influenced by the electrodes electrical properties (low frequency regime) and the cell medium (high frequency regime). An electrode covered with a confluent cell layer shows a shift to higher impedance (solid) allowing to assess parameters as cell-substrate distance, membrane capacitance and the permittivity of the cell layer.

the cell culture section for keratinocytes. A baseline was recorded for $1 \mathrm{~h}$ with only 400 $\mu \mathrm{l}$ medium filled in the chambers and under standard culture conditions in an incubator. Afterwards, the medium was removed and 75,000 cells in $400 \mu$ FAD medium were inserted in every well. Roughly $30 \mathrm{~h}$ after culture, the medium was changed to FAD ${ }^{+}$ (high calcium) medium to allow junction formation. As controls, one well was left with only medium and one cell-covered well per cell population was kept with FAD medium. Measurements were performed at 11 frequencies (ranging from $0.0625 \mathrm{kHz}$ to $64 \mathrm{kHz}$ ) and as fast as possible $(\approx 3$ minutes measuring time per well).

For fluctuation analysis, the power spectral density of the detrended impedance signal of each well was calculated individually at steady-state conditions ( $\mathrm{t}>120 \mathrm{~h}$ ) and the slope arising in a double logarithmic plot was fitted.

\subsection{Cell adhesion noise spectroscopy}

Cell adhesion noise spectroscopy is a new method applicable for example for proliferation, adhesion and signaling assays. Parameters as adhesion strength, cytosolic properties and membrane properties can be monitored over time with a spatial resolution smaller than a cell radius 2 .

The core of cell adhesion noise spectroscopy is a silicon chip with nearly 100,000 small integrated electrolyte-oxide metal-oxide-semiconductor field effect transistors. When they

\footnotetext{
${ }^{2}$ based on the video "Venneos - Cell Analysis beyond Vision"
} 
are covered with e.g. living cells, one can describe the system electrically as a core-coat conductor where the medium filled cleft between the cell and the surface is conducting while the basal cell membrane and the solid-electrolyte interface are insulating coats (figure 2.7 A) [176]. The voltage fluctuations of an uncovered sensor origin in JohnsonNyquist noise and thermal fluctuations leading to a characteristic power spectral density (figure 2.7 B) [176]. Through the addition of cells, so called specific cell adhesion noise is added to the spectrum. By analyzing the shape of the added cell adhesion noise, local cell/membrane changes can be detected when measuring the voltage fluctuations on every transistor.
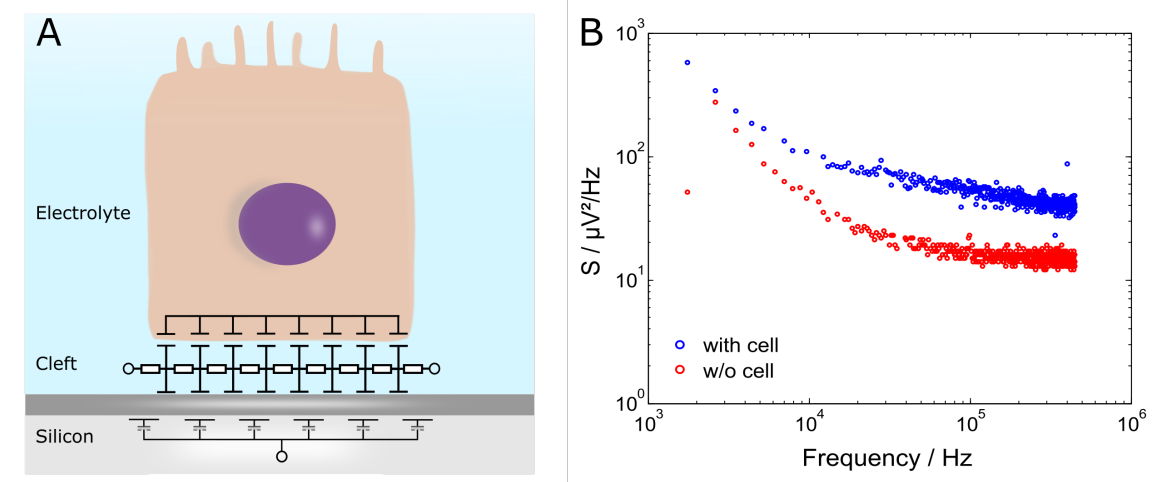

Figure 2.7.: The principle of cell adhesion noise spectroscopy. (A) Voltage fluctuations on cell covered field effect transistors can be electrically described as fluctuations from core-coat conductors. Figure based on [176]. (B) The power spectral density $S$ allows to judge whether a single transistor is cell covered (blue) or uncovered (red) and deduce parameters as adhesion strength or membrane properties (outlier are not shown).

The used setup was a CAN-Q setup (Venneos, Stuttgart, Germany) including chips, software and device which was additionally equipped with a top view Axio Zoom microscope (Zeiss, Oberkochen, Germany). The chips were cleaned according to the manufacturers protocol. The empty chip filled with only $800 \mu \mathrm{l}$ medium (containing HEPES and P/S) was measured first before adding 120,000 cells in $200 \mu \mathrm{l}$ of the same medium. The chip containing the cells was kept in an incubator at standard culture conditions and only for measurements it was inserted in the CAN-Q device. Noise spectra were collected at different time points for 10 minutes. After $30 \mathrm{~h}$, the old medium in the chip was replaced by fresh medium. 



\section{Results}

In chapter 3.5 results of experiments with keratinocytes are described. All other chapters show results of experiments with MDCK II cells.

\subsection{Impact of culture conditions on MDCK II cells}

MDCK II cells can have different shapes and morphology depending on their state. For example, under long culture, the cells start to transport liquid through their lateral junctions leading to a loss of adhesion of whole cell patches [177]. In this section, several changes in appearance are described and visualized which were found due to specific culture conditions.

\subsubsection{Culture medium as critical factor}

Under normal conditions, the nucleus including nucleoli and the cell junctions can be distinguished quite easily in phase contrast images (figure 3.1 A). However, also irregular junctions, which looked like small blisters between cells, were found during experiments (figure $3.1 \mathrm{~A}^{\prime}$ ). It was observed that in a confluent petri dish, this small blisters formed earlier in tightly packed regions with a high cell density. By adding fresh medium, the blisters at junctions mostly vanished indicating that the freshness of the medium and its supplements was a major factor. If this state was kept too long, and the blisters increased in size, the effect was irreversible, indicating a severe harm to the cells.

Another irregular appearance included inhomogeneities inside the cells (figure 3.1 B). It was found out that amphotericin $B$, which was added in live experiments with unsterile conditions against fungal infections, caused this appearance. Therefore, it is advised to not use this antifungal agent in experiments with MDCK II cells. Possible modes of action are discussed in section 4.1 . 


\section{Results}
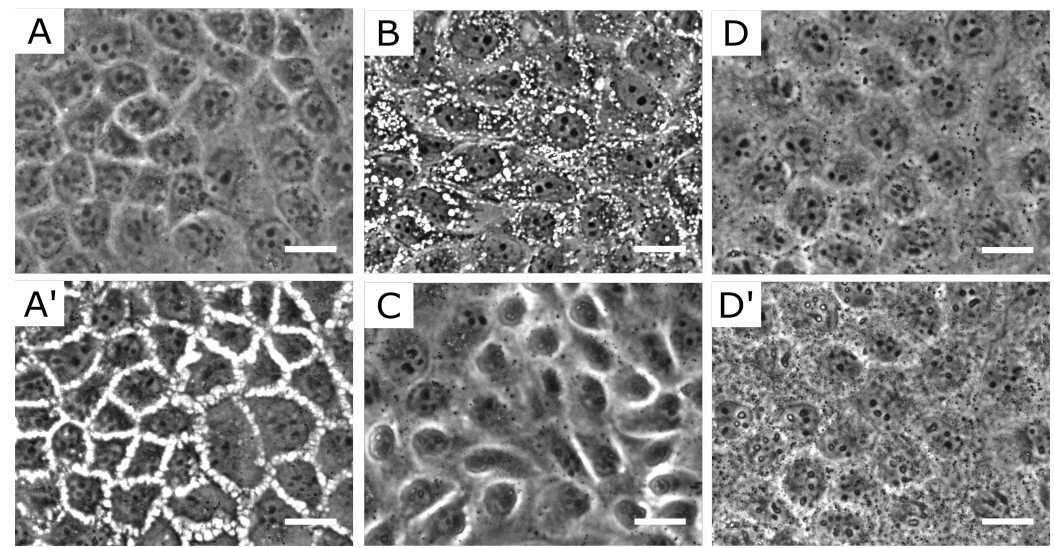

Figure 3.1.: Critical culture conditions during live cell experiments imaged with phase contrast microscopy. (A) normal MDCK II cells and (A') the same cells showing a stress response (B) MDCK II cells treated with the antifungal supplement amphotericin $B$, containing bright roundish spots in the cytoplasm. (C) MDCK II cells cultured for $1 \mathrm{~h}$ without $\mathrm{CO}_{2}$ supply and HEPES buffer. (D) MDCK II cells before and (D') 30 minutes after fluorescence light exposure under standard imaging conditions. All scale bars: $20 \mu \mathrm{m}$.

Another culture supplement in live experiments is HEPES to compensate the missing buffer effect of sodium bicarbonate and $\mathrm{CO}_{2}$ which is present during normal culture in an incubator. Absence of HEPES to samples without $\mathrm{CO}_{2}$ supply resulted in an irregular and more roundish shape of the cells after 60 minutes of culture (figure 3.1 C). Also the cell junctions looked different in phase contrast microscopy indicating the importance of $\mathrm{pH}$ buffering in live cell experiments.

\subsubsection{Impact of light exposure during live experiments}

Another, often neglected, harm to cells in live experiments is photo damage. Fluorophor photobleaching leads to this cell damage, but also high light intensity alone can induce irreversible damage of e.g. the DNA [178]. With every illumination of the fluorophors free radicals are generated and diffuse through the medium. Unlabelled MDCK II cells exposed for $30 \mathrm{~min}$ to standard conditions during fluorescence image acquisition (40x objective, power of $60 \mathrm{~mW}, 600 \mathrm{~ms}$ exposure time, 2 frames per minute, the shutter was closed between image acquisition) already showed drastic changes (figure 3.1 D') compared to the state before light irradiation (figure 3.1 D). As a result of the irradiation, 
the cytosol looked less homogeneous and fragmented and also nucleoli appeared altered. For phenol red, a common cell culture medium supplement to indicate $\mathrm{pH}$ changes, it is known to fluorescence at an excitation wave length of $440 \mathrm{~nm}$ [178]. In this experiment, medium without phenol red was used but the damage was still visible. One reason might be the presence of riboflavin and tryptophan in the culture medium [179] but also the addition of HEPES leads to an increase in cytotoxicity [180]. Therefore, the lowest possible amount of light exposure and a careful observation of cell behavior is inevitable in live cell experiments.

\subsubsection{Temperature dependent behavior of MDCK II cells}

Furthermore, temperature sensitivity of cells could lead to biased results when temperature is not well controlled during experiments. The data and images shown in this section were acquired and processed by Linnea Hoheisel during her bachelor thesis at the Faculty of Chemistry, University of Göttingen.

\section{Microscopy observations at different temperatures}

Firstly, the cells were optically characterized with phase contrast microscopy after 2 hours of incubation at different temperatures. A change in appearance was detected between $44{ }^{\circ} \mathrm{C}$ and $45{ }^{\circ} \mathrm{C}$ (figure $3.2 \mathrm{~A}$ ). At $44{ }^{\circ} \mathrm{C}$ and below the cell layer looked homogeneous, however, at higher temperature, local irregularities appeared. In close-ups (figure 3.2 B) the irregular shape of single cells became more obvious and some cells seemed to form blebs or elongate in one direction. All in all, the layer integrity got disrupted when incubated at temperatures higher than $44{ }^{\circ} \mathrm{C}$ for 2 hours. At lower temperatures, the cells seemed to show irregularities in the cytoplasm as seen in figure $3.2 \mathrm{~B}$. At room temperature $(\mathrm{RT})$, small blisters resembling these appearing after amphotericin $B$ treatment were observed inside the cell. Already at a temperature of $25^{\circ} \mathrm{C}$ this effect nearly completely vanished and the cells appeared very similar to the ones kept at $37^{\circ} \mathrm{C}$. These findings showed that MDCK II cells are very sensitive to temperature changes and obvious shape changes can already be observed with phase contrast microscopy. To gain further insights in the cells' response to temperature changes, actin and ezrin which are both important players in cell mechanics, were fluorescently labeled for these culture 


\section{Results}

conditions.
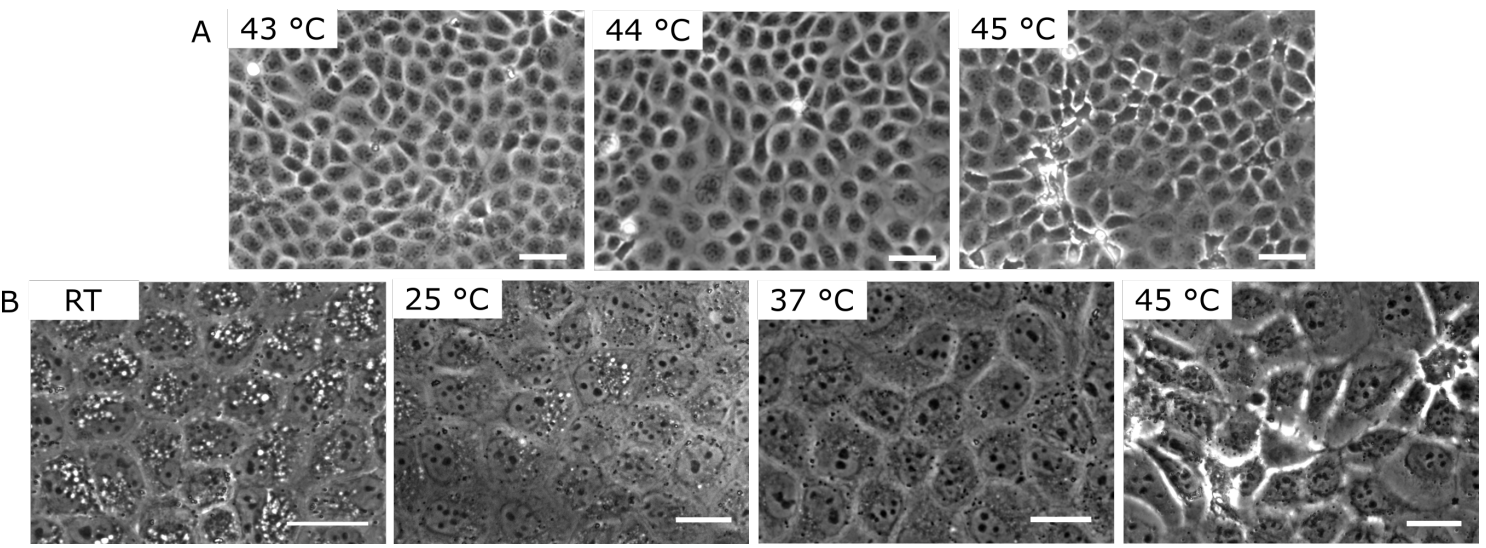

Figure 3.2.: Phase contrast images of MDCK II cells incubated for $2 \mathrm{~h}$ at different temperatures. (A) A change in morphology can be seen at a temperature between $44{ }^{\circ} \mathrm{C}$ and $45^{\circ} \mathrm{C}$. The layer integrity gets disrupted at these temperatures. Scale bars: $50 \mu \mathrm{m}$. (B) Magnifications show that single cells reveal irrgeluarities in the cytoplasm at low temperatures as room temperature (RT) and a very abnormal shape including blebbing at $45{ }^{\circ} \mathrm{C}$. Scale bars: $20 \mu \mathrm{m}$.

Striking changes were seen basal for MDCK II cells. Stress fibers were most pronounced at $30{ }^{\circ} \mathrm{C}$ and $37^{\circ} \mathrm{C}$ (figure 3.3) and the actin appearance was very similar at these temperatures. At room temperature only fragments of stress fibers were visible and round aggregates appeared.

Furthermore, at elevated temperatures $\left(44^{\circ} \mathrm{C}\right)$ no stress fibers were seen at the basal side. However, it seemed that the lateral actin cortex was more enhanced at these temperatures. This demonstrated that MDCK II actively remodel cytoskeletal components upon temperature change, which suggests that temperature does also affect cell mechanics.

Ezrin, as a major contributor in actin-membrane attachment, did not show obvious changes in the appearance at the apical side of the cells (figure 3.4). In contrast, ezrin revealed an enhanced localization at lateral junctions at high temperatures. This nicely agreed with the observation of increased actin at junctions at high temperatures. At low temperatures, the appearance was the same as for $37^{\circ} \mathrm{C}$.

In conclusion, the cell actively reacted to temperature changes by remodeling inner structures, but to a different extent depending on the structure. Further, we hypothesized that this remodeling is accompanied by changes in the cells' mechanics which was tested next. 

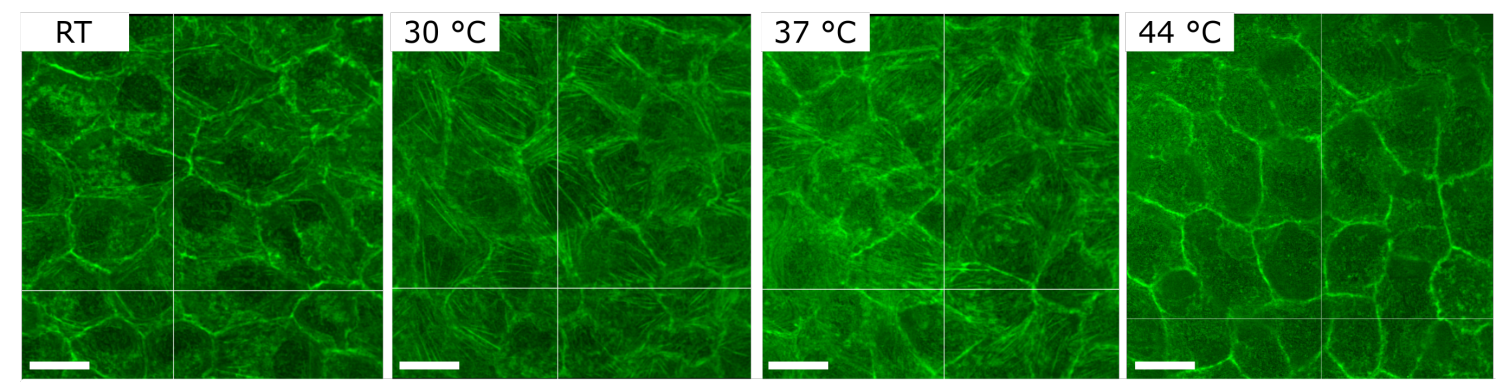

Figure 3.3.: Confocal images of MDCK II cells cultured for $2 \mathrm{~h}$ at different temperatures show a difference in actin appearance. Actin stainings (green) show proper stress fibers at the basal plane only at mid temperatures. At room temperature (RT) only a few fragments appear while at higher temperature none can be seen. At $44{ }^{\circ} \mathrm{C}$, lateral junctions seem more pronounced. Scale bars: $20 \mu \mathrm{m}$.
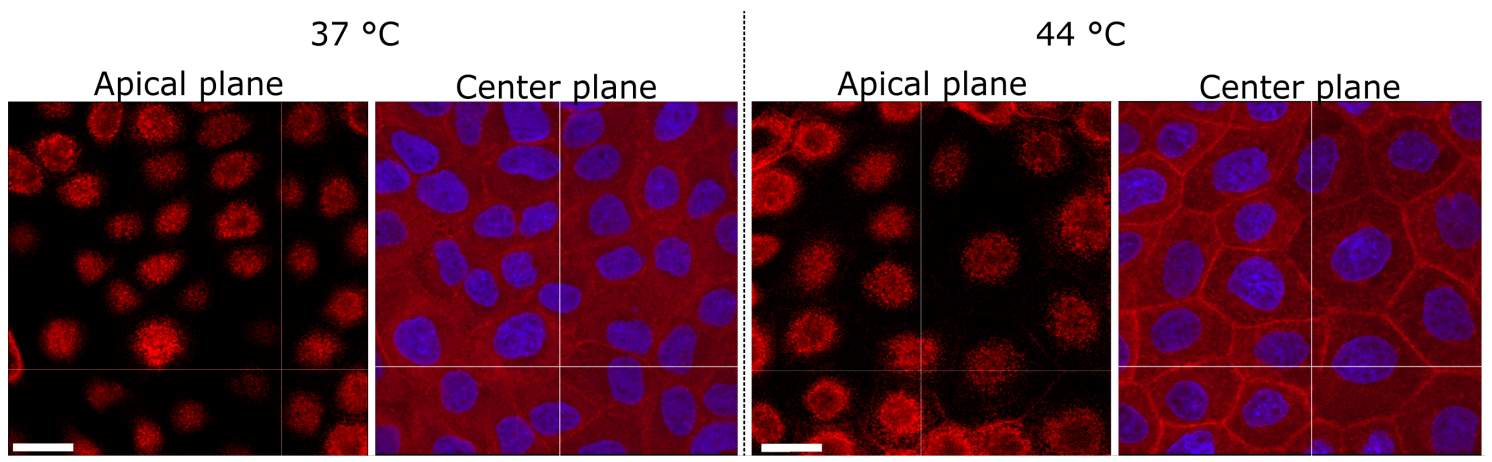

Figure 3.4.: Confocal images of MDCK II cells cultured for $2 \mathrm{~h}$ at different temperatures and stained for ezrin at different z-planes. At high temperatures, ezrin is lateral enhanced at the junctions in the center of the cell. In the apical plane, the ezrin appearance does not change with increasing temperatures. Scale bars: $20 \mu \mathrm{m}$.

\section{Mechanical changes upon temperature change}

To determine mechanical changes upon different temperatures, MDCK II cells were incubated for roughly $1 \mathrm{~h}$ in a cell culture incubator at the desired temperature before being placed in the AFM heating setup and measured at 2 different spots, giving roughly $90 \mathrm{~min}$ and $120 \mathrm{~min}$ as total incubation time.

The mean tether force $F_{\text {tether }}$ showed a strong dependency on temperature (figure 3.5 $\mathrm{A}$, left). In the range between room temperature and $45^{\circ} \mathrm{C}$, the tether force decreased with increasing temperature. The mean force at $25{ }^{\circ} \mathrm{C}$ was more than $100 \%$ larger compared to the mean force at temperatures above $40^{\circ} \mathrm{C}$. A closer look at the cumulative probabilities of the measurement at $90 \mathrm{~min}$ incubation time showed that the whole distribution was shifted to lower values with roughly $70 \mathrm{pN}$ being the minimal tether 


\section{Results}

force at $23{ }^{\circ} \mathrm{C}$ and the largest measured tether force at temperatures above $40{ }^{\circ} \mathrm{C}$ (figure $3.5 \mathrm{~A}$, right). With this finding, the tether force showed the most pronounced effect upon temperature change.

The apparent area compressibilty modulus $\tilde{\mathrm{K}}_{\mathrm{A}}$ (figure $3.5 \mathrm{~B}$ ) was also decreasing with higher temperature. However, the value at $25{ }^{\circ} \mathrm{C}$ seemed to be an outlier. A similar behavior was observed for the apparent pretension $\tilde{\mathrm{T}}_{0}$ but here the outlier was not larger but smaller as expected (figure 3.5 C). A tendency to lower mean values with increasing temperature can also be found in this parameter. Looking at the cumulative probabilities and ignoring the outlier at $25{ }^{\circ} \mathrm{C}$, the overall trend of smaller values at higher temperatures was confirmed for $\tilde{\mathrm{K}}_{\mathrm{A}}$ and $\tilde{\mathrm{T}}_{0}$. For $\tilde{\mathrm{K}}_{\mathrm{A}}$ the population was also shifted while for $\tilde{T}_{0}$ mostly the appearance of larger pretensions (around $0.3 \mathrm{mN} / \mathrm{m}$ and higher) was largely reduced at higher temperatures while the lower boundary of measured pretensions was similar for all temperatures.

All in all, the measurements at the two different time points showed the exact same trends even though different areas were measured, pointing to a general and longerlasting mechanical change. Summing up, this analysis showed that indeed not only internal structures but also mechanical parameters are affected by temperature changes. A reason for the outlier at $25{ }^{\circ} \mathrm{C}$ might be a phase change in the membrane and will be further discussed in chapter 4.1. All these results presented in section 3.1 showed, that MDCK II cells are very sensitive to culture conditions and that critical observation is a prerequisite for generating reproducible results.

\section{Temperature control efficiency}

The microscopy observations described in section 3.1.3 were acquired after incubation in a standard cell culture incubator while for mechanical measurements the temperature was controlled with a petri dish heating system supplied by the AFM manufacturer (figure 3.6 A). During data acquisition, inconsistent results appeared which led to a temperature check of the cell medium which was kept in the AFM petri dish heating system. Figure 3.6 $B$ shows that there was a discrepancy between set point and actual medium temperatures at different positions in the petri dish under measurement conditions. This finding should be kept in mind when judging the AFM experiments. However, taken this finding into account, it even supports the hypothesis that already small temperature changes largely 

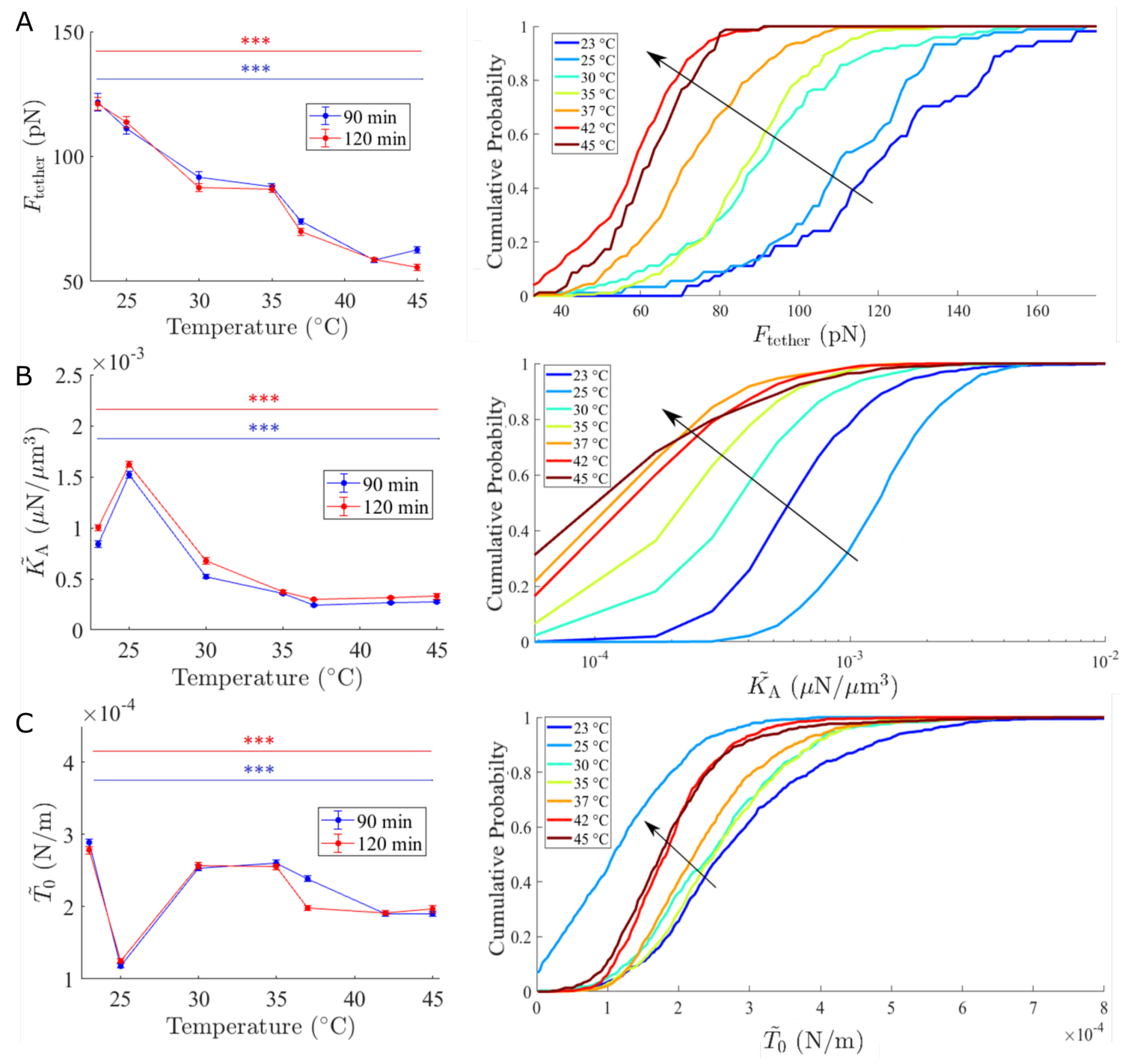

Figure 3.5.: Cell mechanics changes upon different temperatures. (A) The tether force $F_{\text {tether }}$ shows the strongest change with decreasing force for increasing temperature. For (B) $\tilde{K}_{A}$ and (C) $\tilde{T}_{0}$ the same trend is seen but the decrease is less pronounced. The data at $25^{\circ} \mathrm{C}$ represents an outlier of the trend. Left: mean of population for two different total incubation times including standard error of the mean. Right: cumulative probability distributions for all temperatures and an incubation time of 90 minutes. Figure by Linnea Hoheisel. [181]

affect the mechanical state of cells. If set point temperatures would have been reached also in the middle of the petri dish, this presumably would have led to larger changes in the cells' mechanics. 

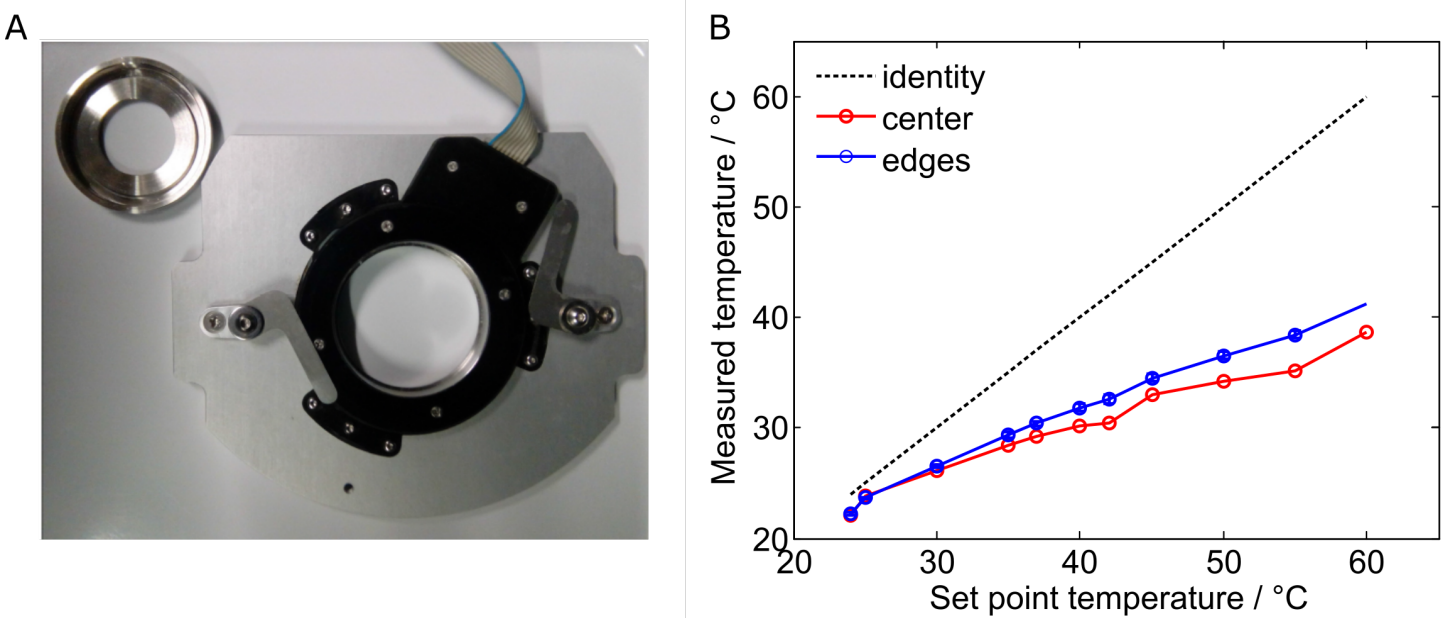

Figure 3.6.: Heating efficiency in the used AFM compatible heating system. (A) The heating system supplied by the AFM manufaturer heats an inserted metal adapter (top left) to the set point temperature and controls the temperature at this component. A petri dish is inserted in this metal adapter for experiments. (B) The set point temperature (black dashed line) can not be reached at the edges (blue, mean and standard deviation of all 4 edges) or in the center (red) of the medium filled petri dish.

\subsection{Growth and onset of collective behavior of MDCK II cell layers}

\subsubsection{Growth behavior of a MDCK II cell layer}

Firstly, the large-scale formation of a MDCK II cell layer was observed with cell adhesion noise spectroscopy. The growth of the cell layer was followed from the time point of cell seeding to the full coverage. To highlight the area covered by cells, the integral of the power spectral density of every measuring pixel is color coded.

Single round cells were barely detectable but small islands were clearly distinguishable within 50 minutes after seeding (figure 3.7). These small islands stayed local on the time scale of 10 minutes indicating a low migration rate. However, a significant growth of cell patches was observed within this time period (white circle in figure 3.7). Over time the cell clusters grew and coalesced to large cohesive clusters. This showed the cells tendency to form stable cell clusters: Once contact was established, cells did not separate from the cluster anymore but stayed local. After $\approx 70$ hours a full coverage was nearly reached.

Next, a closer look at the early formation of cell junctions with fluorescence microscopy 


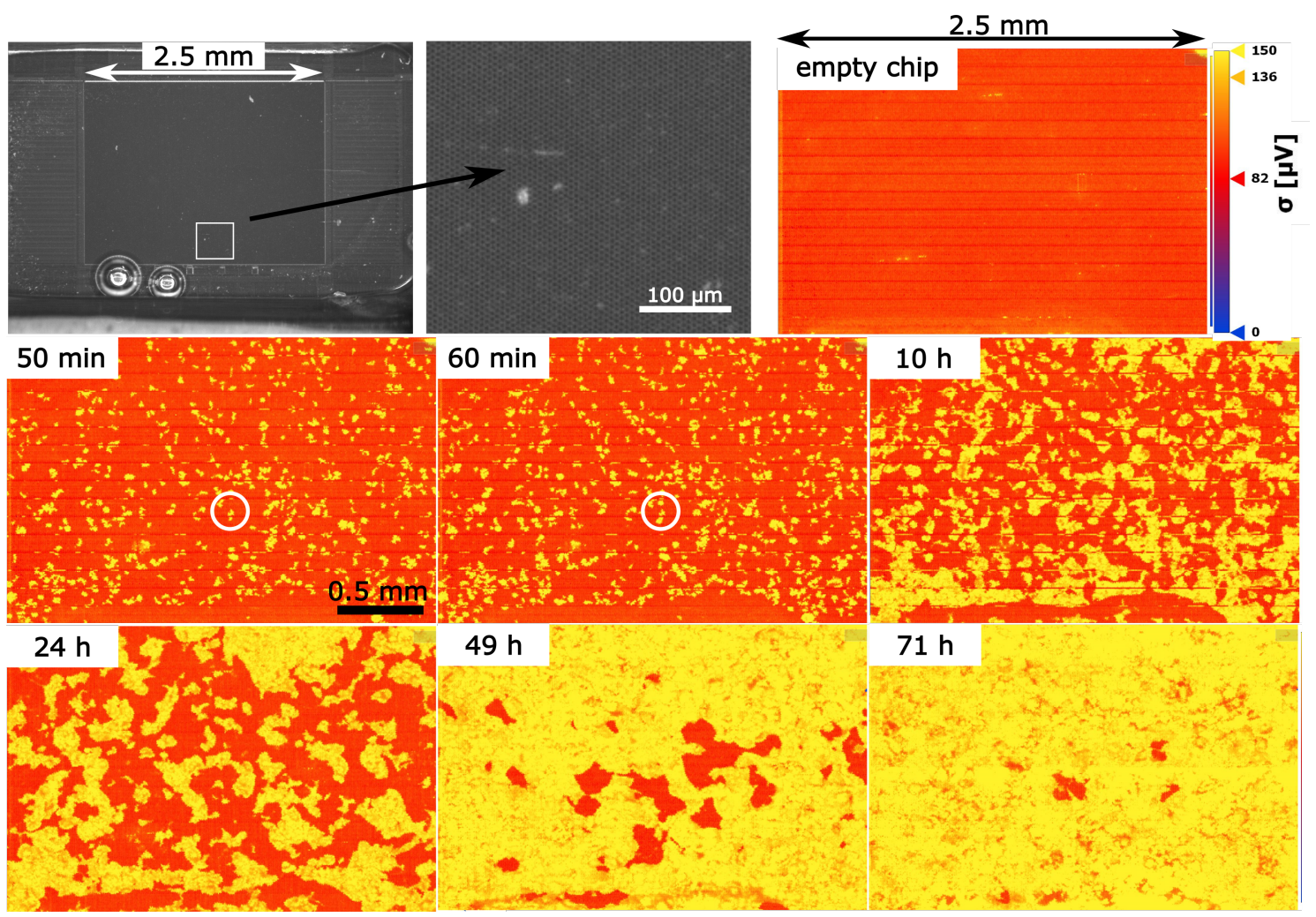

Figure 3.7.: The formation of an MDCK II cell layer observed with cell adhesion noise spectroscopy. The setup allows to measure a large area $\left(1.6 \times 2.5 \mathrm{~mm}^{2}\right)$ with a comparable small pixel resolution of $6.5 \mu \mathrm{m}($ zoom $)$. In a cell experiment, the formation and growth of small clusters very early after seeding (white circles) to the nearly confluent cell layer after $71 \mathrm{~h}$ hours can be detected. The integrated power spectral density allows to judge the pixel coverage and visualize the growth of the cell layer. Line defects visible in the early adhesion and the empty chip are due to miscommunication from the chip to the device and only appear sporadically.

was performed. This is the onset of collective behavior in cell clusters which is crucial for MDCK II cells.

\subsubsection{Early cell-cell junction formation in a growing cell layer}

Sample preparation and image recording for results shown in this section were performed by Philipp Schröder at the Faculty of Chemistry, University of Göttingen [182].

During the establishment of a fully confluent layer, proper cell-cell junctions have to form which ensure polarity in cells but also communication between the cells. As seen in the section above, growing MDCK II layers firstly form small islands before these coalesce 


\section{Results}

to larger structures. Therefore, at a low seeding density of 10,000 cells $/ \mathrm{cm}^{2}$ only small islands were visible while at a high density of 70,000 cells $/ \mathrm{cm}^{2}$ a nearly confluent layer existed already after 1 day of culture.

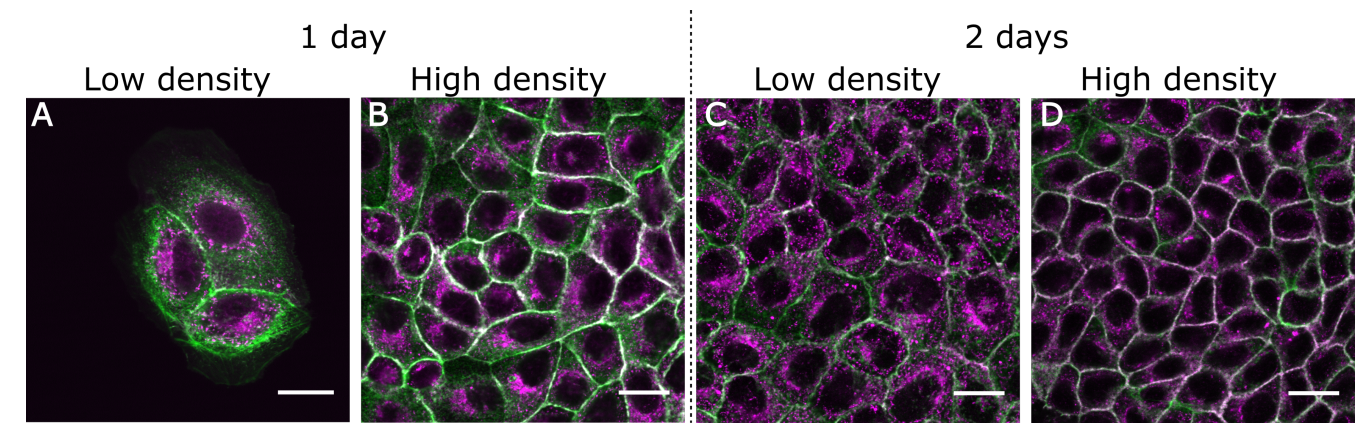

Figure 3.8.: Confocal images of MDCK II cells stained for E-cadherin (magenta) and actin (green) at different seeding densities and culture times. (A) Low seeding densities $\left(10,000 \mathrm{cells} / \mathrm{cm}^{2}\right)$ lead to small islands 1 day after culture where E-cadherin is mostly localized in the cytoplasm. (B) At a higher cell density $\left(70,000\right.$ cells $\left./ \mathrm{cm}^{2}\right)$ after the same culture time, E-cadherin is located at cell-cell junctions (white). (C) In low density seeded cells a weak accumulation of E-cadherin at the junctions after 2 days of culture is observed. (D) The least accumulation of E-cadherin in the cytoplasm is visible after 2 days of culture of densly seeded cells. All scale bars: $20 \mu \mathrm{m}$.

Comparing E-cadherin appearances of cells seeded with these densities and cultured for 1 and 2 days, large differences became visible. In low densities and 1 day of incubation, Ecadherin was mostly localized in the cytoplasm and only sparsely at the junctions (figure 3.8 A). In more densely seeded cells, an accumulation of E-cadherin at the junctions became visible but still a cytoplasmic signal was present (figure $3.8 \mathrm{~B}$ ). Comparing this result to a low seeding density after 2 days of culture, the E-cadherin signal at junctions under the latter conditions was less pronounced. The least cytoplasmic signal was seen in densely seeded cells after 2 days of culture.

A different picture was seen for ZO-1, an important protein in tight junctions. Already in small cell islands ( 1 day of culture, low seeding density) ZO- 1 was highly accumulated at the junctions (figure $3.9 \mathrm{~A}$ and $\mathrm{B}$ ). The signal got even more pronounced, when a higher cell density was seeded (figure $3.9 \mathrm{C}$ and D). This showed that ZO-1 was exclusively found at the junctions in this very early state. Tight junctions form a barrier and are known to maintain surface polarity by separating basal parts from lateral parts [183]. This polarity allows the cell to have different protein compositions but also differences in lipid mixtures at these membrane parts [183]. This can be demonstrated with the glycoprotein 135 (GP 135), which is known as a very early apical marker protein [184, 185]. As seen in figure 

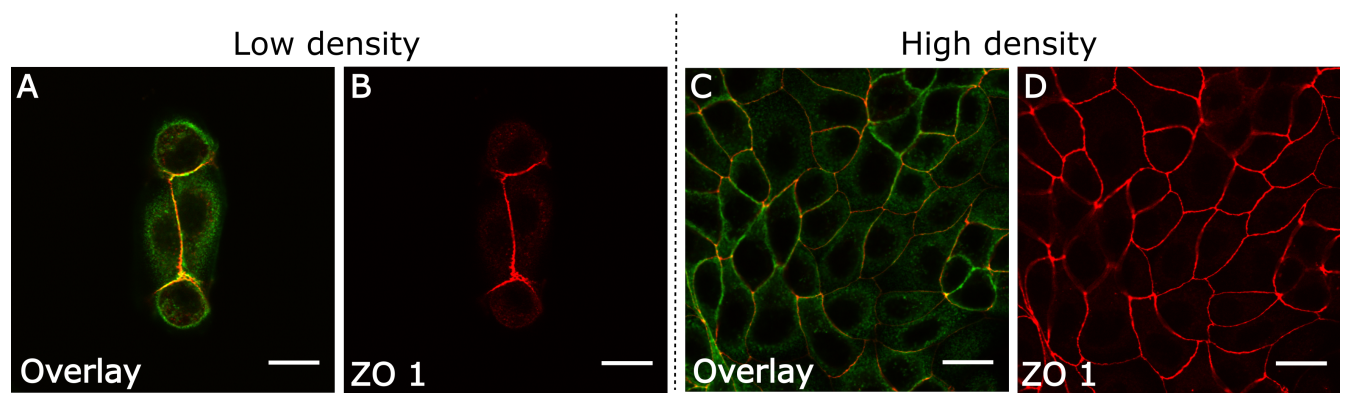

Figure 3.9.: Confocal images of MDCK II cells stained for ZO-1 (red) and actin (green) after 1 day of culture. (A) Already in small cell islands an accumulation of ZO-1 at cell-cell junctions is visible while (B) in the cytoplasm nearly no signal is detectable. (C) In densly seeded cells the signal at the junctions is even stronger. (D) ZO-1 is exclusively localized at the junctions. All scale bars: $20 \mu \mathrm{m}$.

3.10 A in the xz-plane, GP 135 exclusively localized at membrane parts not touching the substrate, already in the early state where only islands have formed. When the cells were denser (high density, 1 day of culture), GP 135 was found exclusively at the apical membrane, the lateral membrane was devoid of it (figure $3.10 \mathrm{~B}$ ). Colocalization with ZO-1 showed that exactly the position of ZO-1 determined the separation of different membrane compositions, which was demonstrated here with the staining for GP 135 (figure $3.10 \mathrm{C}$ ). This implied that also apical-basal polarity is established very early in a newly forming cell layer.
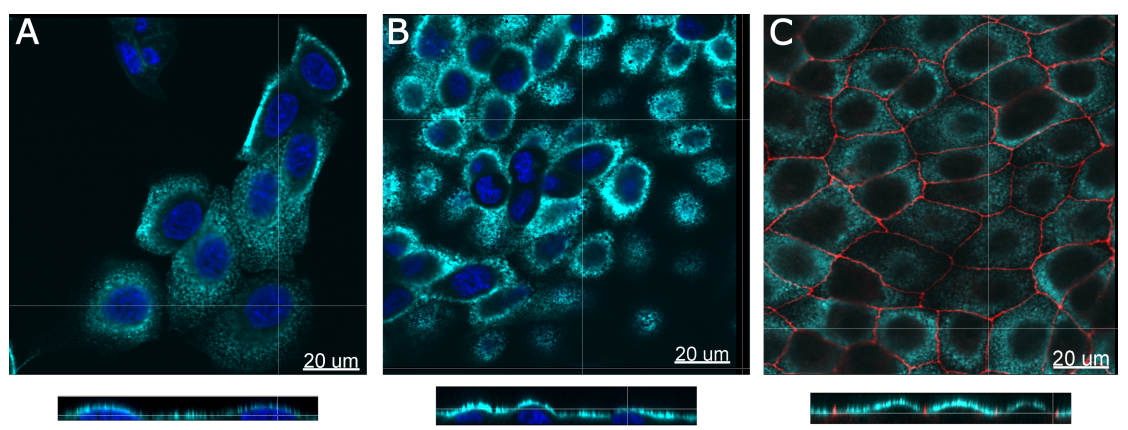

Figure 3.10.: Confocal images showing apical polarity in cells with different seeding densities. Xy images and $\mathrm{xz}$ sections of MDCK II cells at different seeding densities after 1 day of culture demonstrate the localization of the apical marker GP 135 (cyan) with respect to ZO-1 (red). Nucleus shown in blue. (A) Already at a low seeding density GP 135 exclusively localizes at membrane parts not touching the underlying substrate. (B) With a high seeding density it becomes obvious that also the lateral membrane is devoid of GP 135. (C) Co-staining with ZO-1 shows that the position of ZO-1 and the localization of GP 135 give a consistent picture.

Looking at the three dimensional shape of the cells revealed that in early adhesion (high 


\section{Results}

density, 1 day of culture) the cells were extended in the xy-plane and very flat (figure 3.11 A). After 2 days of culture the cells were much smaller due to cell division which takes place roughly once per day for MDCK II cells (figure 3.11 B). Also the cell height was increased and a lateral extended cell-cell junction was formed building large adhesive areas.

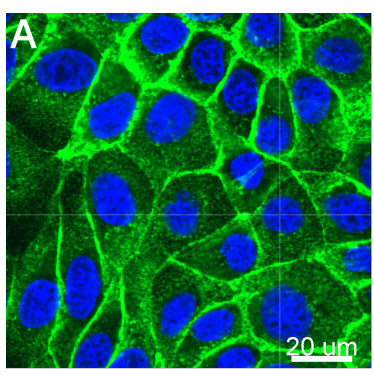

$5 \mu \mathrm{mI}$

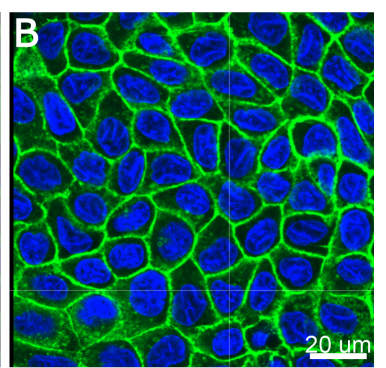

$8 \mu \mathrm{m} T$

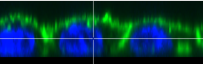

Figure 3.11.: Actin distribution in densly seeded cells. Xy images and xz sections of densly seeded cells after (A) 1 day and (B) 2 days of culture stained for actin (green) and the nucleus (blue). During the second day of culture the cells decrease in area in the xy plane but increase in height, building up a distinct lateral membrane.

Summing up, large amounts of E-cadherin localized on an overall longer time scale to the newly forming lateral junction site than ZO-1.

Additionally, Ojakian postulated that the early localization of GP 135 to the apical domain is due to a tight coupling to the actin scaffold [185]. Indeed, also the actin cortex rearranged over the time of culture growth. Basal, the actin accumulation at the cell borders was not very distinct and irregular within 1 or 2 days of culture. Laterally, the actin signal got more distinct and also the cells shape became more regular as identified by the appearance of the actin cortex (figure 3.12).

These results indicated that the cell layer and the junctions are constantly rearranged during growth and even after reaching confluency. The formation of vertically elongated junction sites enables the cells to increase the contact area to neighbors. This allowed the cells to form clusters of adhesion proteins and hence stable cell-cell contacts. Especially the load bearing elements as actin and E-cadherin largely rearranged upon layer growth. 

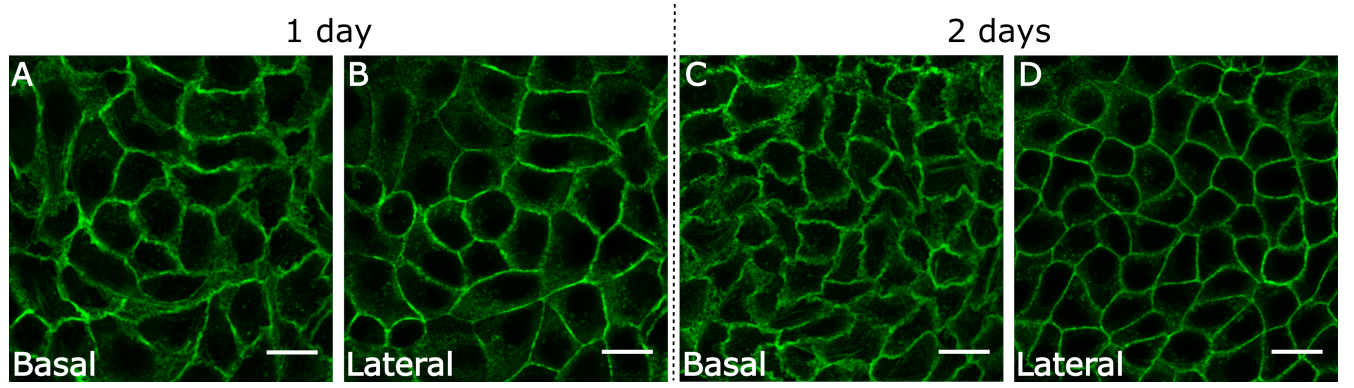

Figure 3.12.: Actin filaments in densly seeded MDCK II cells after different culture times. (A) Basal actin gives a spread signal with irregular borders. (B) At a higher lateral z-position where the junctions are located the cells show a distinct actin signal. (C) 2 days after culture the basal cell shape is still irregular but (D) laterally, the actin signal is even more distinct and the cell shape is more defined. All scale bars: $20 \mu \mathrm{m}$.

\subsubsection{Controlling cell-cell junction geometry using micro patterning}

\section{Quality of micro patterning}

Next, the idea was to interfere with this growth process and to create defined junction lengths with the micro patterning technique $\mu$ PIPP (microscale plasma-initiated protein patterning). A regular pattern of PLL-PEG was printed on a glass surface and the sample was then incubated with collagen I. The resulting pattern can be seen in figure $3.13 \mathrm{~A}$ demonstrating the general success of the method. Detailed analysis of line scans gave varying results (figure $3.13 \mathrm{~B}$ ): On the one hand, collagen could not cover the PLL-PEG coated areas leaving these areas non-adhesive for cells, as expected. On the other hand, collagen levels were not always elevated at the areas without PLL-PEG. In this case, increased levels of collagen were found at the borders of the PLL-PEG coated areas.

\section{Distribution of cells on the collagen pattern}

Firstly, it was observed that cells did not adhere and spread on solely PLL-PEG coated areas but remained in a round state (figure $3.14 \mathrm{~A}$ ). This showed that the PLL-PEG coating was suitable to completely block cell adhesion which was in accordance with other techniques from the literature that used PEG [186]. On a pattern including adhesive areas, individual cells did adhere and spread (figure $3.14 \mathrm{~B}$ ). Moreover, the actin cytoskeleton adapted to the geometry of adhesive areas (figure 3.14 C). During layer 


\section{Results}
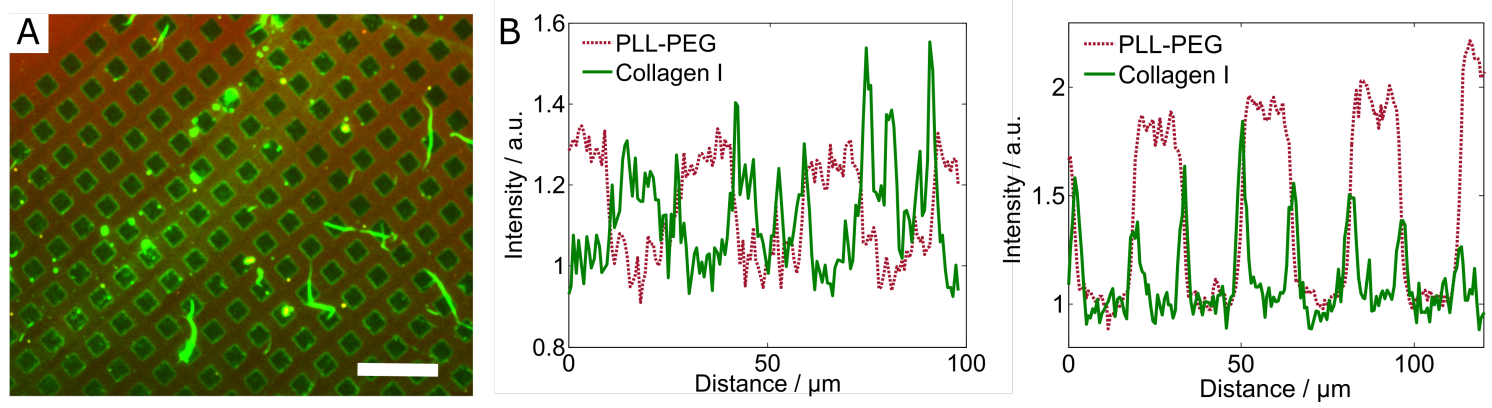

Figure 3.13.: Pattern obtained with the micro-patterning method. (A) A regular pattern of PLL-PEG (red) and collagen (green) is achieved. Scale bar: $100 \mu \mathrm{m}$ (B) Two examples of fluorescence intensity line scans demonstrating that collagen is not homogeneously distributed on the PLL-PEG-free areas while the PLL-PEG distribution is very distinct.

growth, neighbor contacts got established and the cells started to spread to neighboring adhesive areas (figure $3.15 \mathrm{~A}$ ). This led to a loss of order. At borders of the establishing cell layer, the actin still adapted the shape of the underlying substrate pattern but in cells completely encircled by other cells this distinct actin pattern got lost (figure 3.15 B). After establishment of a full layer, no order in cell arrangement across the pattern was detected (figure $3.15 \mathrm{C}$ ). Even larger non-adhesive areas were fully covered with cells (figure $3.15 \mathrm{C}$ ). At these sites, several neighboring cells were not able to adhere to the substrate but could only form junctions to neighboring cells. This showed that micropatterning works for individual isolated cells but it is not suitable to control junction length or position in a MDCK II cell layer grown to confluency: if cells are allowed to form cell-cell contact, adhesion to the substrate does not matter anymore. This finding well accompanies studies with freely-suspended cell monolayers [126, 187] and highlights the importance of cell-cell junctions in an epithelial cell layer. Their presence and function is essential for collective behavior and seems to dominate the cells behavior in homeostasis.

\subsection{Classical wound healing essays to create large wounds}

In order to disturb layer homeostasis and monitor changes in the remaining cells, different methods were tested. Classical wound healing essays to create large defects were tested 

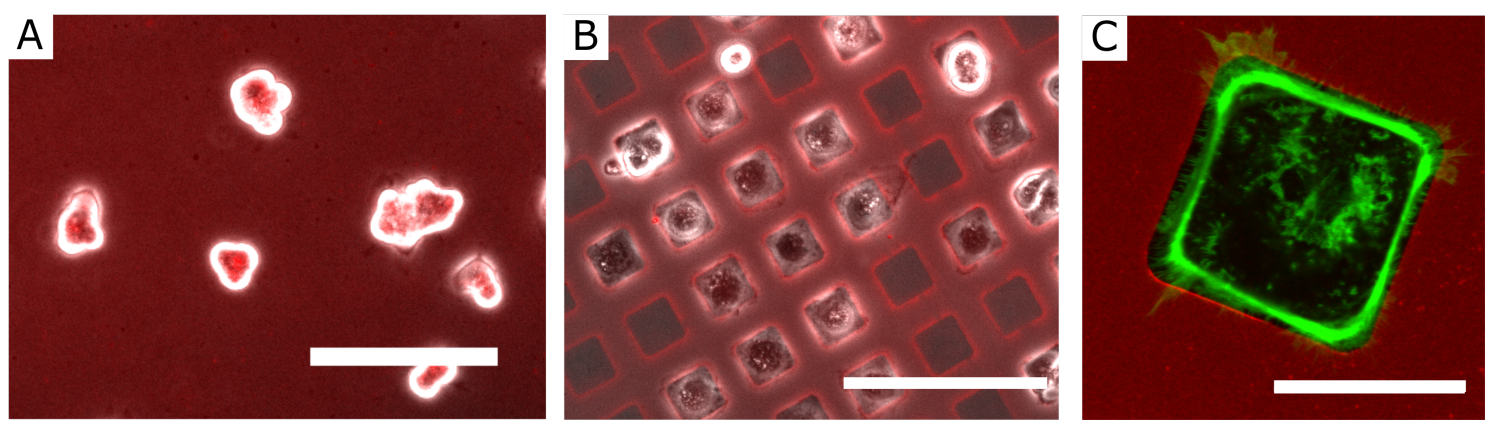

Figure 3.14.: Isolated MDCK II cells on a micropattern. (A,B) PLL-PEG distribution (red) overlaid with a phase contrast image demonstrating (A) the blocking of PLL-PEG and (B) cell arrangement of isolated cells on adhesive areas. (C) Actin staining (green) overlaid with PLL-PEG distribution (red) showing the adaptation of the actin cytoskeleton to the pattern in isolated cells. (A,B) Scale bars: 100 $\mu \mathrm{m}$, (C) scale bar: $20 \mu \mathrm{m}$.
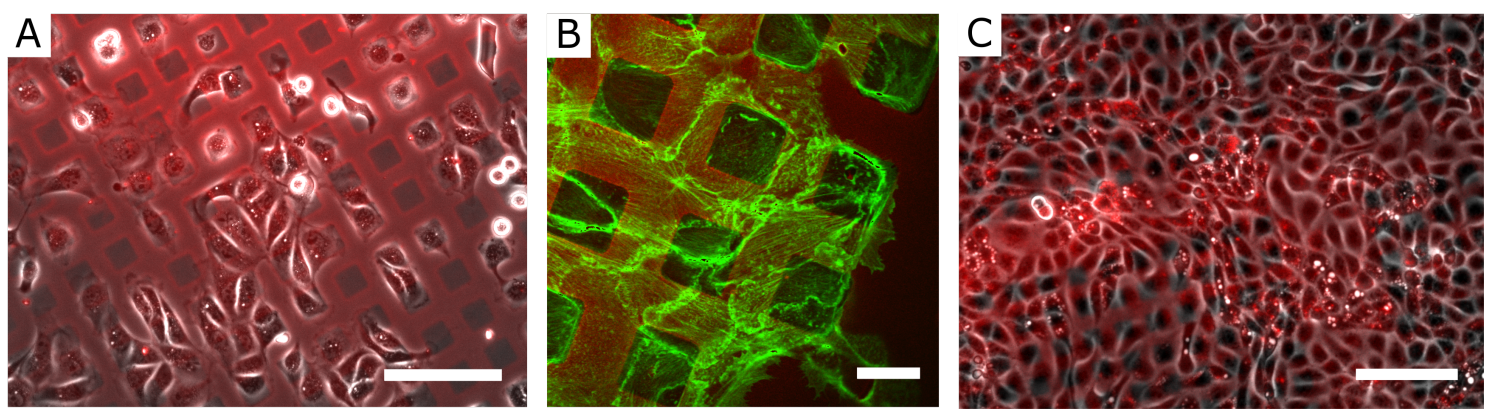

Figure 3.15.: MDCK II cells with neighbor contact on a micropattern. (A) PLL-PEG distribution (red) overlaid with a phase contrast image of cells with neighbor contact. Upon contact, cells elongate to neighboring adhesive areas. Scale bar: $100 \mu \mathrm{m}$ (B) Actin staining (green) overlaid with PLL-PEG distribution (red) showing the loss of defined actin distribution upon neighbor contact. Scale bar: 20 $\mu \mathrm{m}$. (C) Overlay of phase contrast image of a confluent cell layer with fluorescence image of the PLLPEG pattern (red) demonstrating the full coverage of the surface and the loss of order. Scale bar 100 $\mu \mathrm{m}$.

first.

\section{Irregular wound edges after a scratch assay}

A commonly used method to induce a macroscopic wound is to destroy parts of the layer through a scratch with a sharp tip, called scratch assay. Here, a cell free gap with a width of about $500 \mu \mathrm{m}$ was created (figure $3.16 \mathrm{~A}$ ). The defect edges were massively affected using this method. On the one hand, cells neighboring the free gap were detached from the substrate but still adhered to other cells (figure $3.16 \mathrm{~B}$ ). Free parts of the layer floated in the medium and the cells state was far away from normal culture conditions. 

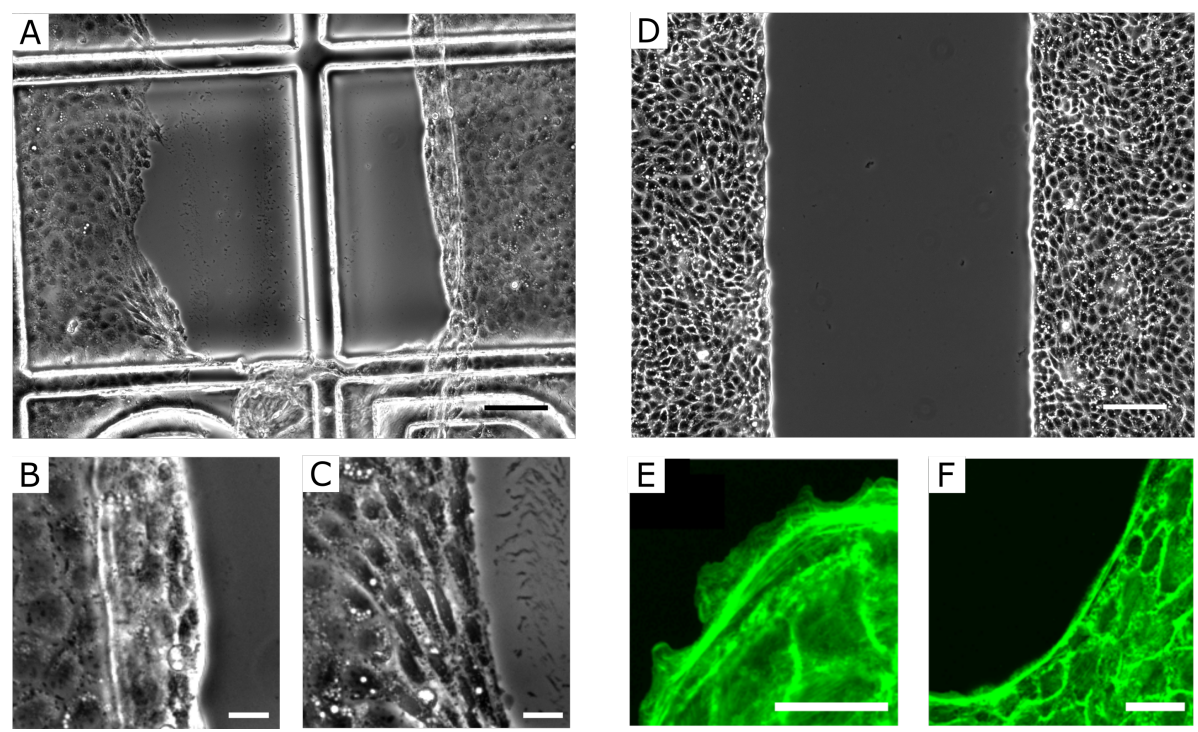

Figure 3.16.: Wound induction via classical wound healing essays. (A) Multicellular wounds created by a scratch assay exhibit (B) front cells without substrate adhesion or (C) which were exposed to massive mechanical shear stress. (D) Culture insert induced wounds show intact cells but to close the wound, mechanisms as (E) lamellipodia formation or (F) contractile actin cables are used leading to very diverse cell populations. ( $A, B, C, D)$ phase contrast images, $(E, F)$ fluorescence migrographs with actin stained in green. Scale bars: (A,D) $100 \mu \mathrm{m},(B, C, E, F) 20 \mu \mathrm{m}$

On the other hand, where the layer was still attached to the substrate, the cells in the first rows were deformed heavily in one direction (figure $3.16 \mathrm{C}$ ). Most probably the deformation was in direction of the applied scratch and appeared when neighboring cells were removed with the tip. All in all, this technique did indeed create a wound in confluent cell layer, however, the cells neighboring the defect were massively altered by wounding. This experiment also demonstrated the strength of cell-cell junctions. Even under these harsh influences and shearing forces the junctions hindered the cells from disconnecting directly from the layer. Several rows of neighboring cells were affected before some cells got ripped apart from the layer.

\section{Use of culture inserts}

Culture inserts were used which prevented the cell layer from covering the whole surface of a culture dish. When removing the insert, a macroscopic wound of $500 \mu \mathrm{m}$ was created which was subsequently closed by the cells. Initially, the cells at the wound margin were rather small and round in comparison to the bulk cells (figure $3.16 \mathrm{D}$ ). 
After $3 \mathrm{~h}$ of migration different wound healing mechanisms became obvious. On the one hand, lamellipodia were formed (figure $3.16 \mathrm{E}$ ). According to Ravasio et al. these can be found at regions of positive curvature of the migrating layer [141]. Here, thick actin fibers were interrupted and flat cell protrusions formed. On the other hand, thick multicellular actin cables surrounded the wound margin (figure $3.16 \mathrm{~F}$ ). According to the literature, these can be mainly found at regions with negative curvature [141]. With this cable and subsequently via actomyosin contractility, the wound margin is pulled forward. Because of these two mechanisms, the cells' shape and contractile patterns were very diverse. The cell layer was under constant movement and remodelling. Therefore, a comparison to a fully established layer would be misleading. That is why single-cell manipulation was applied next, where only small disturbances to the cells' homeostasis were inserted.

\subsection{Single-cell defects in a confluent MDCK II layer}

Major parts of the sections 3.4.1-3.4.5 are published in the article Single-cell defects cause a long-range mechanical response in a confluent epithelial cell layer [136]. The text is taken either word-by-word or is closely related. Figures are rearranged but kept in the original style. Laser ablation experiments were performed together with D. Kong (Institute for Developmental Biochemistry, Medical School, University of Göttingen).

\subsubsection{Characteristic closure of a single-cell defect}

In order to induce a large harm to a single cell in a confluent layer, namely a complete single-cell failure, micropipette action was used (see figure 2.2). A change in phase contrast indicated a successful treatment. As response, the wounded cell was lifted and extruded from the cell layer (figure 3.17 A). Major driving mechanism was the contraction of the supracellular actomyosin ring acting essentially as a purse string which was seen 30 minutes after wounding (figure $3.17 \mathrm{~B}$ ). This was similar to the actin cable described in section 3.3 in classical macroscopic wound healing essays, but here the actin formed a complete circle and surrounded the whole defect cell. Finally, the wound was closed after approx. 100 minutes (figure $3.17 \mathrm{C}$ ). The typical rosette formation was seen in this process due to slight junction elongation towards the defect center (figure 


\section{Results}

3.17 D). During the first 10 minutes after wounding, the wound size stayed roughly constant. This was in accordance with findings from others showing that during this time frame rho-mediated actomyosin ring assembly happens prior to wound shrinkage [154]. During wound closure, staining of the apical marker protein GP135 revealed that
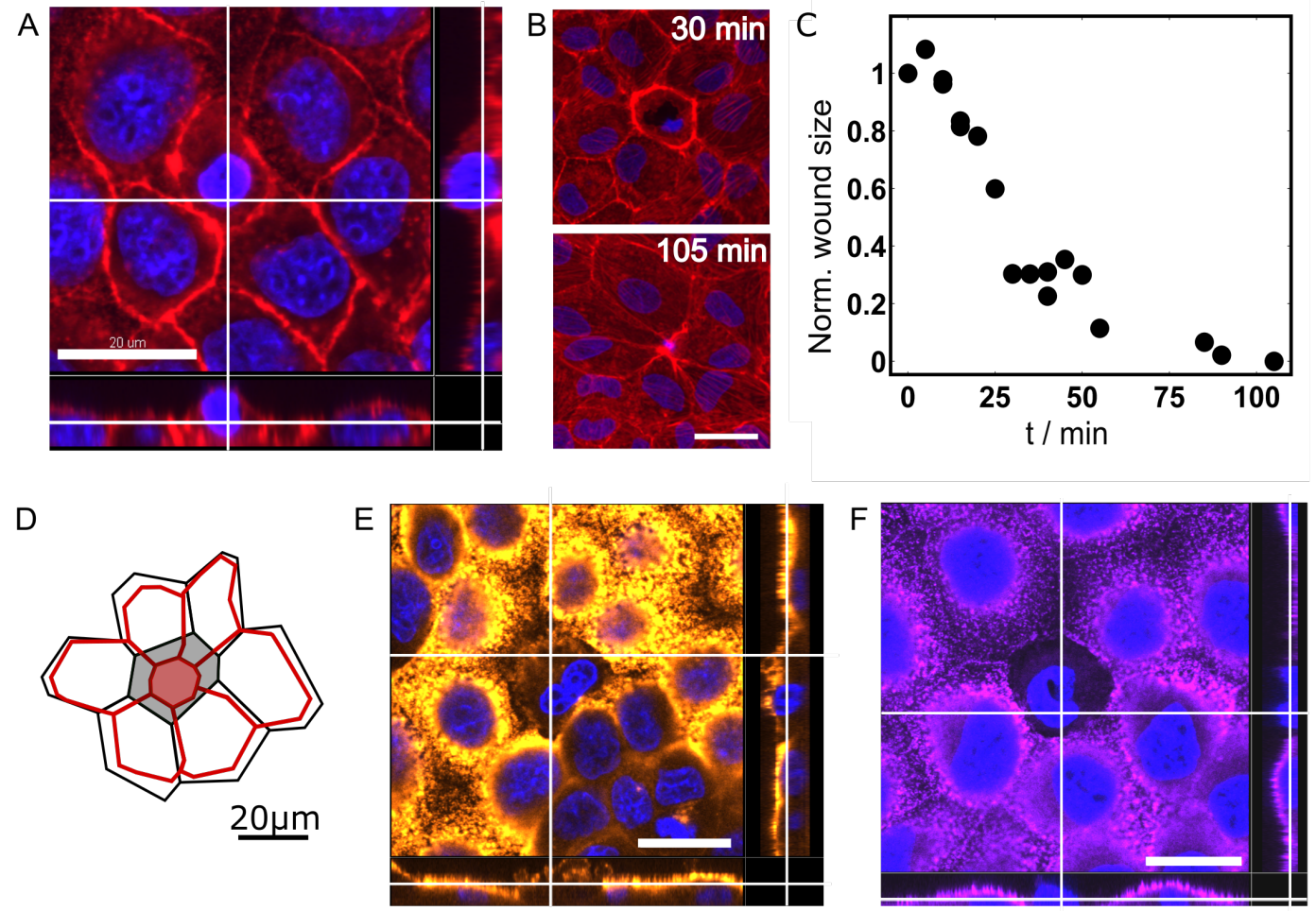

Figure 3.17.: Closure of a single-cell defect. (A) Confocal image including $x z$ and $y z$ projections of a wounded cell which is increased in cell height before extrusion (red: actin). (B) Fluorescence micrographs $30 \mathrm{~min}$ and $105 \mathrm{~min}$ after wounding reveal an accumulation of actin filaments (red) surrounding the wounded cell. The filaments remain visible even after the wound is closed. (C) Typical time trace of the normalized wound size (normalized to initial area of the cell) during closure. (D) Example of cell shapes before (black, thin) and 50 min after wounding (red, thick) of the center cell showing the typical rosette formation. (E) Confocal image including $x z$ and $y z$ sections showing that the apical membrane (stained for GP135, orange) is wrinkled in the wounded cell. (F) Confocal image including $x z$ and $y z$ sections demonstrating that the wounded cell is nearly devoid of ezrin (purple). Scale bars: $20 \mu \mathrm{m}$, nuclei are shown in blue.

the apical membrane of the defect cell was folded and showed lots of blebs (figure 3.17 E). Moreover, ezrin was absent in the wounded cell, indicating a loss of coupling between the membrane and actin cytoskeleton (figure $3.17 \mathrm{~F}$ ). 


\subsubsection{Mechanical properties of cells neighboring the single-cell defect}

Force indentation experiments were conducted on cells adjacent to the wound and more far away to find out whether cells mechanically react to a neighboring single-cell wound. The apparent area compressibility modulus $\tilde{\mathrm{K}}_{\mathrm{A}}$ of cells did not show a resilient dependency on the cells position, namely neighboring the defect or in an intact cell layer during seven independently performed experiments (appendix, figure A.1). Hence, the regulation of recruiting membrane reservoirs was not inherently altered by the closing process. Here, one also has to consider that the values varied over four orders of magnitude. As the measured $\tilde{K}_{A}$ value is dominantly measurable at high strain, i.e. in large indentation depths, the forces recorded there might also be influenced by cell organelles, like the nucleus and hence depended on the position of the indentation on the cell.

In contrast, the apparent pretension $\tilde{T}_{0}$ was significantly different in cells neighboring a wound than in cells of an intact cell monolayer (appendix, figure A.2). Combining all independent experiments $(\mathrm{N}=7)$, a significant increase in the apparent pretension was visible for cells directly adjacent to a defect compared to control cells in a homogeneous layer (figure $3.18 \mathrm{~A}$ ). This result was also reproducible, when the same cells were measured two times: firstly undisturbed and secondly, after inducing a single-cell wound in the center of the measurement area (figure $3.18 \mathrm{~A}^{\prime}$ ). To rule out that the increase in $\tilde{\mathrm{T}}_{0}$ at the border of the wound was due to perturbations by the cantilever indentation, exactly the same spot was measured two times with the same time delay needed for a wounding process but without inducing any defect in the cell layer. No significant shift was measurable leading to the conclusion that the induced wound is responsible for an increase in apparent pretension in cells neighboring and hence closing the single-cell wound (figure $3.18 \mathrm{~B}$ ). To verify that the effect was independent of the way the wound was generated, we also used laser ablation to generate single-cell wounds.

Energies of $\approx 80 \mu \mathrm{J}$ per cell led to the same wounding and closure process as observed for the mechanical ablation. Also after induction of the single-cell defect, an increase in the apparent pretension of neighbors was measurable (figure $3.18 \mathrm{C}$ ). This showed, that the stiffening is an intrinsic process generally occurring in single-cell wound closure.

As stated in section 2.5.2, the cells pretension $T_{0}$ compiles contributions from membrane tension and actomyosin contractility of the cortex attached to the plasma membrane. 


\section{Results}
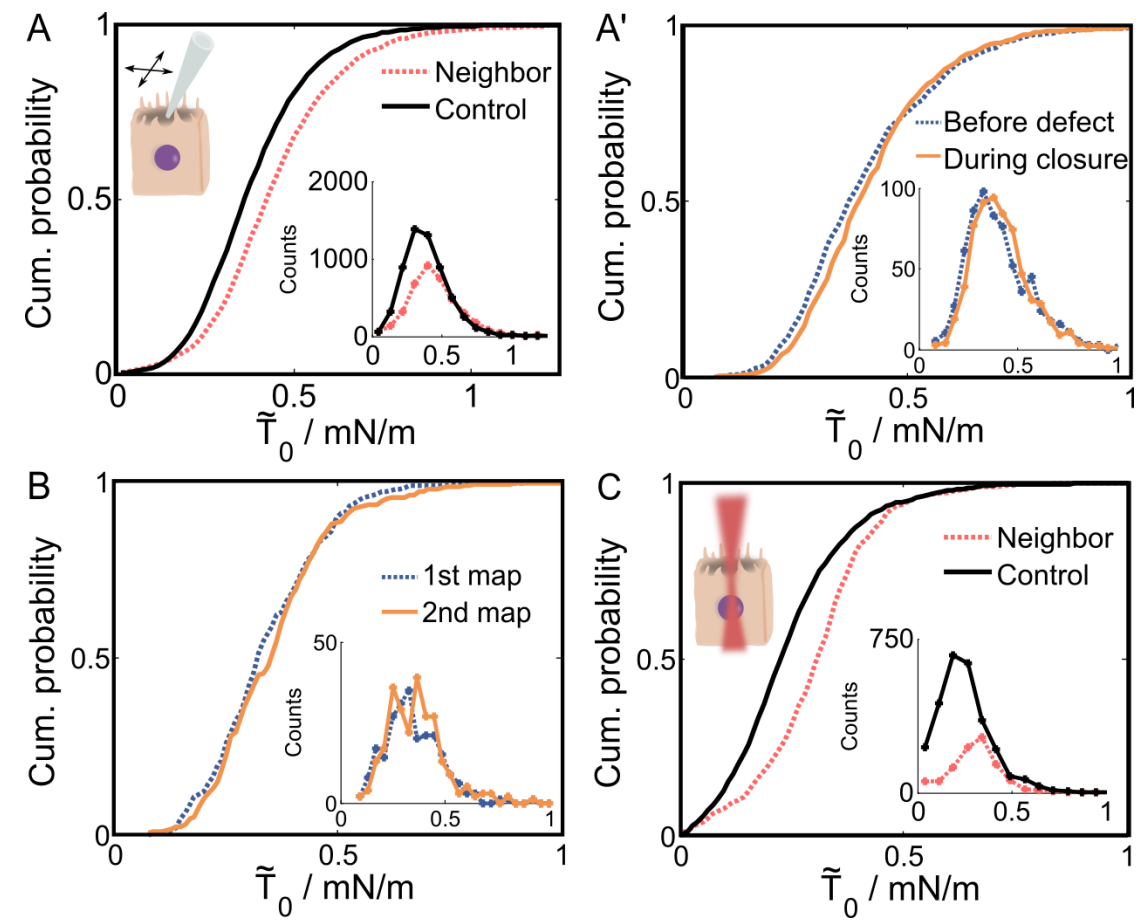

Figure 3.18.: Pretension is altered in cells neighboring a defect. (A) Apparent pretension $\tilde{T}_{0}$ of cells next to a micropipette-induced single-cell wound (red, dashed) compared to control cells in an undisturbed cell layer (black, solid) $(p<0.001)$. Shown is the cumulative probability of $\tilde{T}_{0}$ and the corresponding histogram as an insert. ( $A^{\prime}$ ) Comparing the same cells before any disturbance (blue, dashed) and during closure of a neighboring defect (orange, solid) led to a significant increase $(p=0.034)$. (B) Measuring undisturbed cells twice with the same time delay as needed for wounding did not change the apparent pretension $(p=0.28)$. (C) Defect induction with a laser ablation setup led to the same effect as mechanical micropipette action: cells neighboring the defect (red, dashed) display a higher apparent pretension than control cells in an undisturbed layer (black, solid). $(p<0.001)$.

In order to disentangle the two contributions and to see which one was responsible for the increase in pretension, tether forces were measured upon retraction of the cantilever giving access to the membrane tension.

Tether rupture forces showed no resilient difference between cells neighboring a wound and control cells surrounded by an intact cell layer (appendix, figure A.3). As a consequence, the measured increase of the apparent pretension $\tilde{T}_{0}$ only originated from changes in cortical tension. 


\subsubsection{Mechanical properties of cells neighboring larger wounds}

The question was now, whether the increase in pretension was limited to single-cell wounds where the purse string mechanism prevails or if closing of a larger cell free wound showed the same general trend. Therefore, front cells of an incomplete cell layer (see figure 2.1) exhibiting a clear border and having rather regular cell geometries were mapped (figure 3.19 A). An even larger increase in the apparent pretension was detected in adjacent cells to the border (figure $3.19 \mathrm{~B}$ ). This result showed that even in larger cell free wounds the pretension increased in adjacent cells most likely due to crawling of cells close to the wound and dragging neighbors towards the defect in the cell layer. This was in accordance with findings that cells undergoing the epithelial-to-mesenchymal transition display higher cortical tension of the mesenchymal phenotype [188].
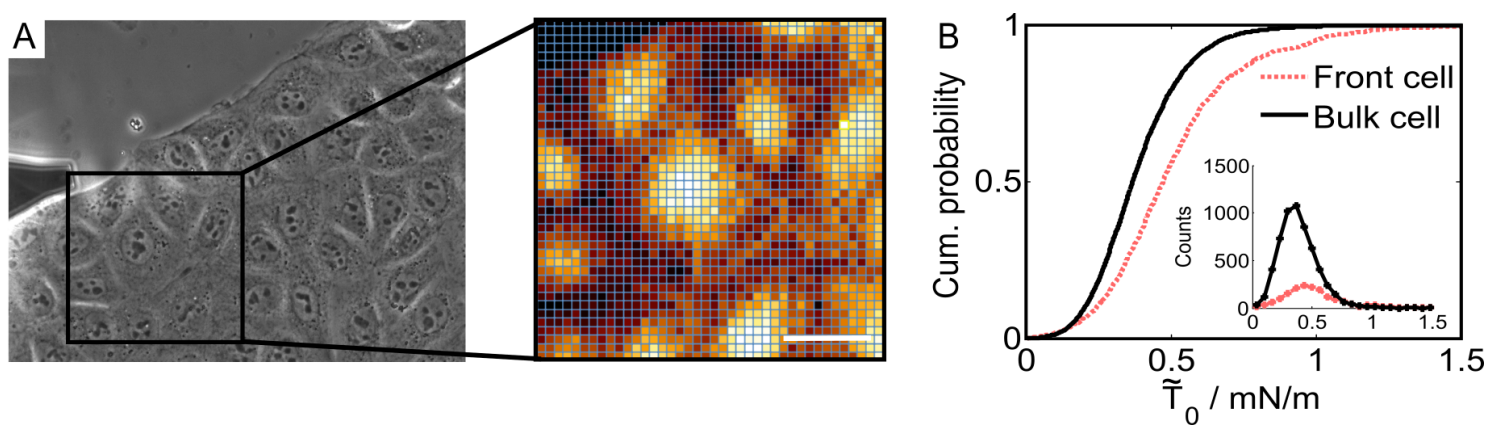

Figure 3.19.: Mechanical investigation of the migration front of an incomplete cell layer. (A) Phase contrast image and AFM force map (height reconstruction). Scale bar: $20 \mu \mathrm{m}$. (B) Apparent pretension $\tilde{\mathrm{T}}_{0}$ in cells at the migration front (red, dashed) compared to bulk cells far away from a border (black, solid) $(p<0.001)$. Shown is the cumulative probability of $\tilde{T}_{0}$; insert: histogram.

\subsubsection{Spatial extent of altered mechanics in the wounded cell layer}

Spatz and coworkers investigated the force correlation of cells as a function of distance from a larger wound and found that up to ten cells away traction forces remain increased [119]. Along similar lines, Trepat and coworkers report that contractile forces are transmitted over supracellular length scales via focal adhesions [151]. Therefore, we asked the question to which extent cells that are not in direct contact to the defect also display altered cortical mechanics due to cell-cell mechanotransduction. It was found that the 


\section{Results}

apparent pretension $\tilde{T}_{0}$ was still increased in 4th row neighbors (named 'neighbor 4'), which were separated by 3 cells, hence several tens of microns away from the defect (figure $3.20 \mathrm{~A}$ ). Also, for the incomplete cell layer a far reaching mechanical effect was measurable. Compared to a fully confluent cell layer with its cobblestone morphology, the cells' shape was not as well-defined under these conditions. Hence, not the degree of neighborhood but distance from the wound margin was considered. Here, even up to $90 \mu \mathrm{m}$ away from the migration front, an increased apparent pretension $\tilde{\mathrm{T}}_{0}$ was found (figure $3.20 \mathrm{~B}$ ). In the case of the single-cell wound, where the increased pretension was less pronounced, the effect already vanished when cells more than $100 \mu \mathrm{m}$ away from the defect were measured before and after inducing the defect (figure $3.20 \mathrm{C}$ ). This means that the information was spread over greater distances than the diameter of a single cell, which is probably due to the strong connectivity of MDCK II cells acting as a mechanical syncytium rather than individual cells (figure 3.20 D). Still, this far reaching mechanical response goes in line with correlation lengths of several cell widths found for migrating MDCK II cells [69, 119]. As the disturbance by a single-cell defect is smaller also smaller spatial extension of the effect was expected. All in all, also cortical mechanics seemed to highly correlate between cells in close proximity most likely provided by cell-cell contacts.
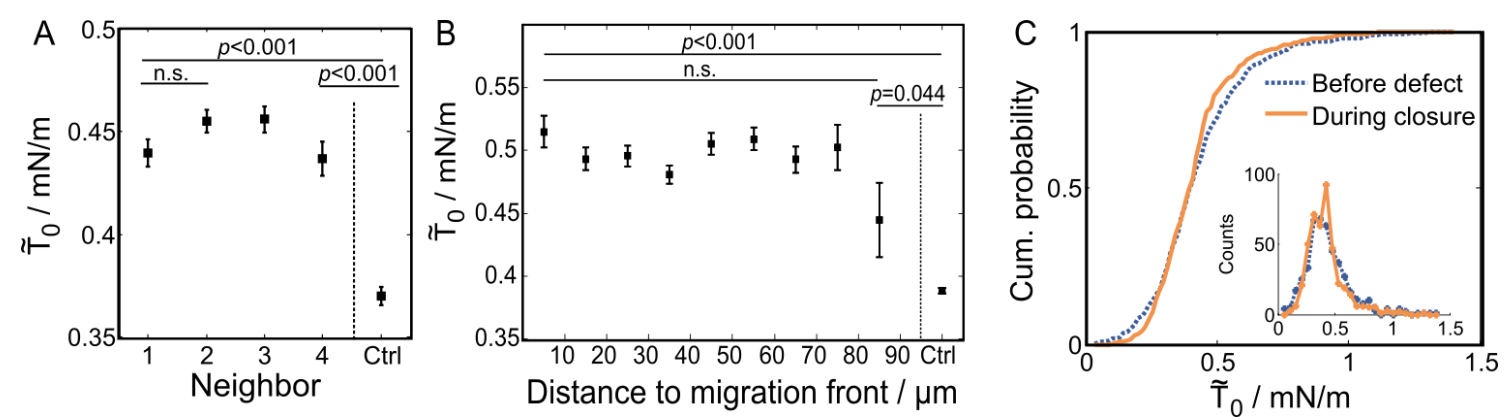

Figure 3.20.: Cellular mechanics as a function of distance from the wound. (A) Elevated apparent pretension $\tilde{T}_{0}$ is also measureable in cells belonging to outer shells of the defect (with 'neighbor 1 ' being the direct neighbor and 'neighbor 4' being separated by 3 cells from the wound) and (B) at larger distances from the migration front of a subconfluent layer displaying clear borders. Shown is the mean \pm standard error. (C) The apparent pretension $\tilde{T}_{0}$ stays constant $(\mathrm{p}>0.05)$ when measuring the same cells before (blue, dashed) and after (orange, solid) inducing a defect in larger distances (more than $100 \mu \mathrm{m}$ away from measuring area). Shown is the cumulative probability; insert: histogram. 


\subsubsection{Constant cell geometries during closure}

Another fact is that mechanical properties of cells as measured by force-indentation are also affected by the cellular morphology such as size and geometry [189]. Therefore, we also investigated size and shape parameters of the cells during wound closure and examined whether simple morphological changes could also account for the observed increase in pretension. The analysis showed that the circularity of cells neighboring a defect stayed constant over time (figure 3.21 A) and also the aspect ratio revealed no strong tendency (figure $3.21 \mathrm{~B}$ ). This showed that the cells do not change their overall shape during single-cell wound closure in a way that would explain the elevation in pretension. However, at around $40 \mathrm{~min}$ after wounding the variations in the aspect ratio of adjacent cells increased. At this stage, the individual cells slightly elongated towards the wounded spot, which was necessary for the typical rosette formation. As these changes were still marginal compared to the overall cell size and very individual for every single cell, a switch in morphology did not explain the obvious increase in pretension. Additionally, the projected area stayed constant over time with only small variations (figure $3.21 \mathrm{C}$ ). This further implied that the area loss during wounding was not compensated by the direct neighbors alone but was a rather cooperative and collective process. Therefore, after the wound was finally closed, also the area loss was collectively compensated by a large number of marginally size changes in more cells than only the five to seven direct neighbors.
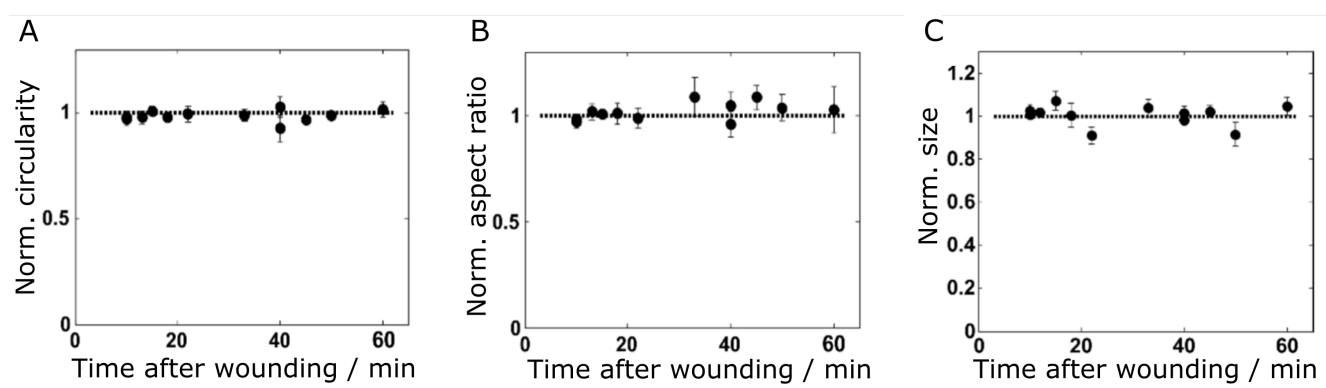

Figure 3.21.: Neighbors of single-cell defects do not change their overall shape. (A) Neither the circularity not $(B)$ the aspect ratio or $(C)$ the size are largely altered during the closure. Induvidual values are normalized to size of wounding before averaging. Shown is the mean \pm SEM for $N=5-7$ cells per data point. 


\section{Results}

\subsubsection{Contributors to the collective closure}

The question arose how these collective behaviors were coordinated between cells. Firstly, calcium signals during single-cell defects were examined. Calcium signaling was already observed after mechanical induction of larger defects [190, 191]. Fluorescence images after addition of calcium indicator and wounding showed two different effects. In one part of the experiments, a traveling calcium wave as described in the earlier studies was seen within the first minute after wounding (figure $3.22 \mathrm{~A}$ ). Radial line profiles originating at the wounded cell showed a gradual intensity decrease in direct neighbors over time (figure 3.22 B). An increase was probably not seen due to the slight time lag between defect induction and start of video recording. Cells in larger distance to the single-cell defect showed their fluorescence intensity maximum at later time points. The wounded cell displayed a constant level.

Another effect which was observed was that only the wounded cell showed an intensity increase within the first minute after wounding (figure $3.22 \mathrm{C}$ ). Line profiles for this case indicated that only the wounded cell (positioned in the middle of the line profile) reached increased levels while the direct neighbors remained a constant calcium level (figure 3.22 D).

All in all, calcium might be an important early signaling mechanism to neighbors in these single-cell defects in MDCK II layers as well. However, mechanical effects were longlasting and other signaling paths contributing at longer time scales have to be activated as well. Therefore, cell junctions coupled to the actin cytoskeleton were investigated.

In E-cadherin GFP transfected cells, a change in signal was observed within the first minutes after wounding. The E-cadherin signal was only increased in the wounded cell (figure 3.23 A). Analysis of line profiles confirmed this finding but also here an increase in junctional E-cadherin could not be seen (figure $3.23 \mathrm{~B}$ ). However, the noise level was quite high and the closure seemed to be not as efficient as in non-transfected cells. This could either be due to the transfection or to the exposure of fluorescence light as discussed in section 3.1.2. That is why non-transfected cells were stained for E-cadherin after wounding and fixation. First an increase in cytoplasmic signal was seen as well while later also junctional E-cadherin exhibited an increased intensity in the wounded cell (figure $3.23 \mathrm{C}$ ). Both effects vanished after roughly 20 minutes. Also changes in junctions further away were not detected. In the case of ZO-1 as marker for tight junctions only 

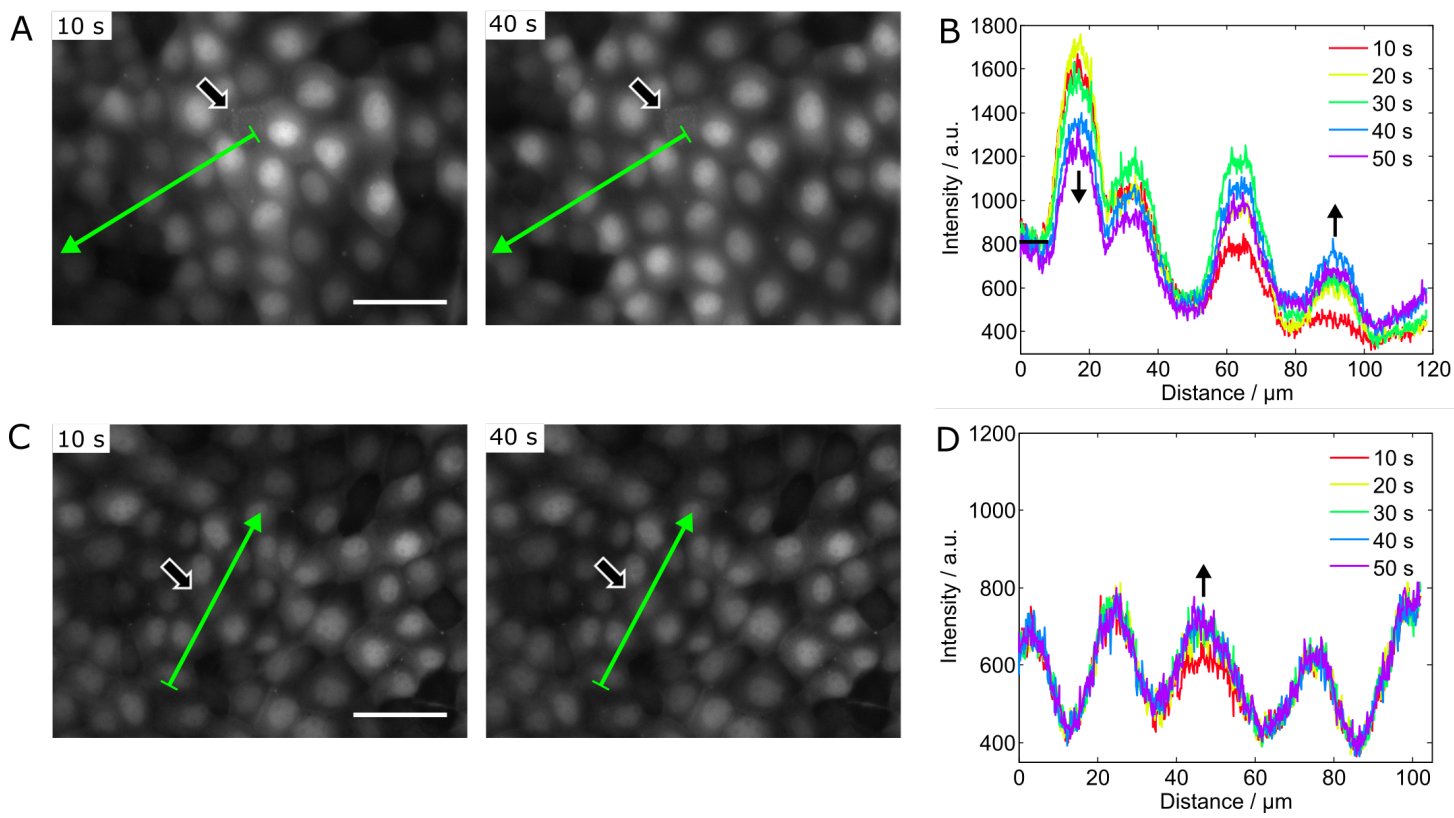

Figure 3.22.: Two calcium signaling characteristics after inducing a single-cell defect. Time indicates the time after defect induction. ( $A, B)$ In some cases a travelling calcium wave can be seen. (A) Fluorescence images of cells treated with a calcium indicator where the wounded cell is indicated by a thick arrow. (B) Fluorescence intensity profile along the green arrow indicated in (A) with the defect cell at the origin. (C,D) In other cases only the wounded cell reveals an increase in intensity. (C) Fluorescence images of cells treated with a calcium indicator where the wounded cell is indicated by a thick arrow. (D) Fluorescence intensity profile along the green arrow indicated in (C). Scale bars: $50 \mu \mathrm{m}$.

slight irregularities in the distribution around the wounded cell were seen during closure (figure 3.23 D). To sum up, it seemed that a change in E-cadherin signals happened suggesting some involvement of adherens junctions during closure. However the effects took place on a shorter time scale compared to the overall closure. Also changes in junctions further away from the defect were not detected. Maybe longer lasting effects around the wounded cell and in cells further away were small and beyond our detection limit.

Lastly, the importance of contractility was tested by the usage of the rho-kinase inhibitor Y27632. During standard closure, a purse string is expected rounding up the cell contours of the wounded cell. In case of inhibition of the rho pathway, no circular accumulation of actin fibers was seen around the wounded cell (figure $3.24 \mathrm{~A}$ ). At the basal side, no stress fibers were detected indicating the successful application of the inhibitor but the wound site also got diminished most probably due to actin polymerization in the 


\section{Results}
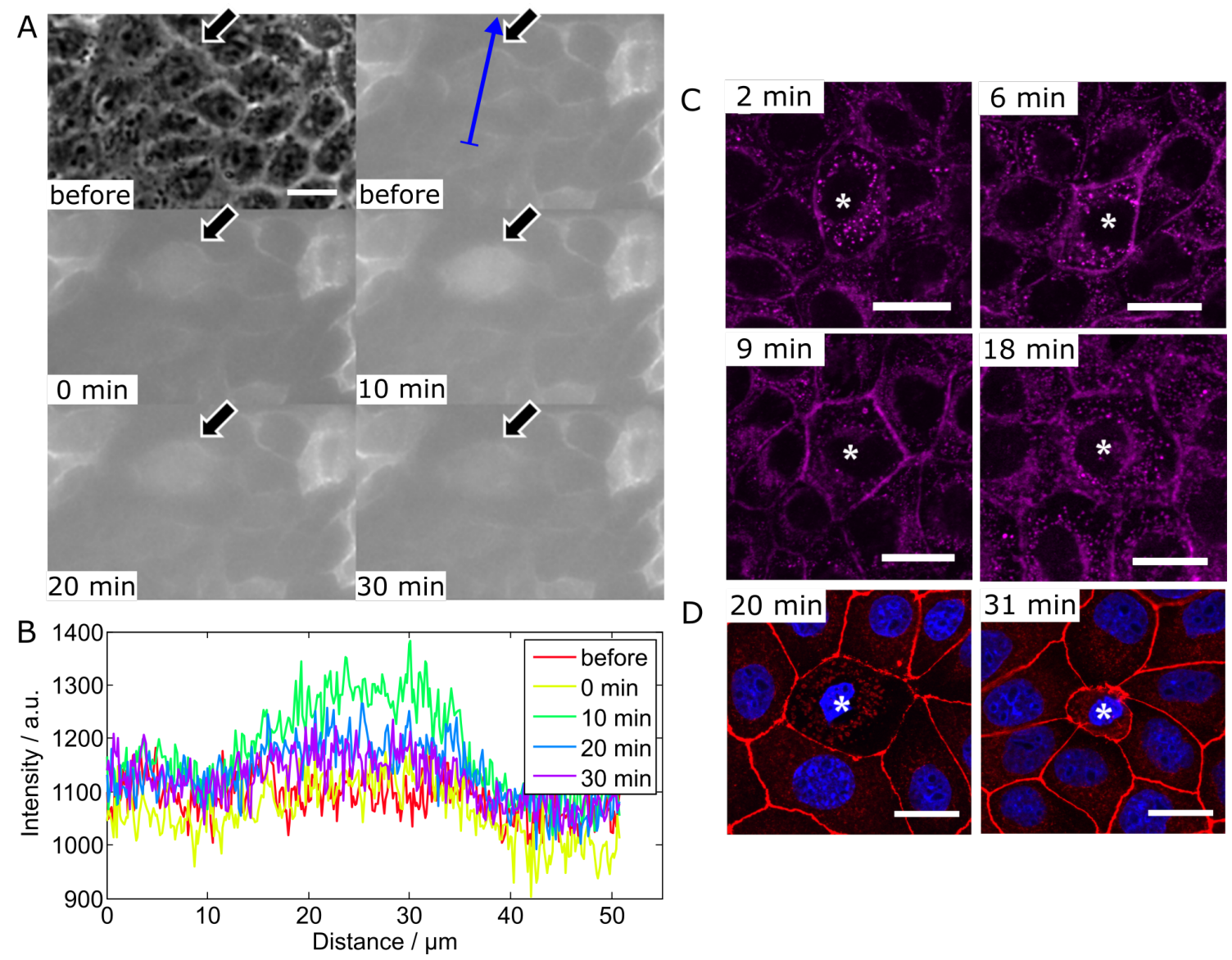

Figure 3.23.: Changes in junctional proteins during closure. (A) Fluorescence micrographs (and one phase contrast) of live imaged E-cadherin-GFP transfected cells at different time points after wounding. The wounded cell is marked with a thick arrow. (B) Line profiles of fluorescence E-cadherin GFP intensity across the wounded cell as marked with ablue arrow in (A) for different time points after wounding. (C,D) Fluorescence micrographs of fixed cells stained for (C) E-cadherin (magenta) and (D) ZO-1 (red) (nucleus shown in blue) at different indicated time points after wounding. The wounded cell is marked with an asterisk. Scale bars: $20 \mu \mathrm{m}$.

surrounding cells.

In general, both mechanisms of actin purse string and lamellipodia formation through polymerization could occur which was demonstrated in one example (figure $3.24 \mathrm{~B}$ ). However, due to the curvature and size of a single-cell wound, the purse string mechanism is highly favored under normal circumstances [141]. Upon inhibition of rho pathway however, purse string closure was impossible.

A detailed understanding of the exact signal transduction mechanism and the general reason for the pretension increase is still missing due to the complexity and contributions 


\subsection{Single-cell defects in a confluent MDCK II layer}
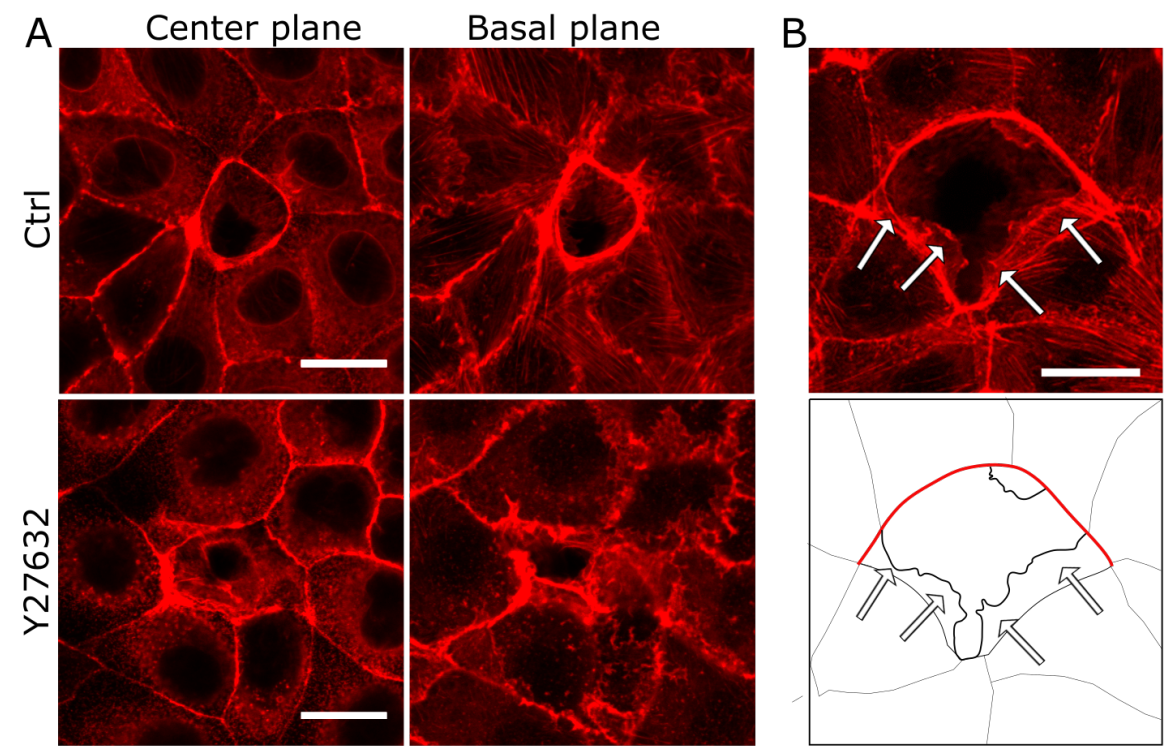

Figure 3.24.: Contractility during wound closure. (A) Fluorescence micrographs showing that upon inhibition of rho pathway, the actin (red) purse string as seen in controls vanishes and the wounded cell does not round up. Actin polimerisation at the basal plane however decreases the wound size upon addition of Y27632. In controls the purse string is active. (B) In one example of a large cell both mechanisms of lamellipodia formation (arrows) and purse string can be seen simultaneously as depicted in the schematic drawing. All scale bars: $20 \mu \mathrm{m}$.

from biochemical and physical effects. However, calcium signaling, E-cadherin reorganization and contractility seemed to be involved in this process of collective wound healing of single-cell defects. 



\subsection{Influence of keratin deficiency on barrier function and mechanical integrity in keratinocyte cell sheets}

Parts of the following section (including figures) are published in the article An intact keratin network is crucial for mechanical integrity and barrier function in keratinocyte cell sheets [192]. Text is taken either word-by-word or is closely related. Most fluorescence microscopy experiments were performed by Fanny Büchau (Institute for Biology \& SIKT, University of Leipzig) which is indicated below the figures.

\subsubsection{Absence of the keratin network leads to altered junction and cytoskeletal architecture}

To further understand how collective behavior is influenced by cytoskeletal contributions, a second cell line was used. These keratinocytes were genetically modified to lack the entire keratin network. Moreover, keratinocytes are of special interest as they are the major cell type in the skin, being directly affected by macroscopic wounding. Earlier studies showed that the absence of the keratin network affects the composition and localization of desmosomes [193]. In this study we found that other types of junctions are also affected. For tight junction proteins as occludin and ZO-1, a disturbed localization at cell-cell junctions was observed $48 \mathrm{~h}$ after calcium addition and the proteins were inhomogeneously distributed along the cell periphery (figure 3.25). In contrast, in WT cells a continuous signal at the cell periphery was present. For claudin 1 and claudin 4 the localization at junctions of $\mathrm{Kty}^{-/}$- cells was even more perturbed and no elevated fluorescence signal at the junctions could be detected. These findings suggest that tight junctions are immature or compromised in $\mathrm{Kty}^{-/}$cells.

In addition to tight junctions, adherens junctions were also affected in $\mathrm{Kty}^{-/}$cells (figure 3.26). The E-cadherin distribution showed an irregular appearance in $\mathrm{Kty}^{-/}$- cells while in WT cells a continuous fluorescence signal at cell-cell junctions was visible. Also p120-catenin localization at junctions was impaired in $\mathrm{Kty}^{-/}$cells, suggesting that the localization of the full adherens junctions complex is disturbed.

Atomic force microscopy imaging confirmed the impairment of junctions (figure 3.27A). 


\section{Results}
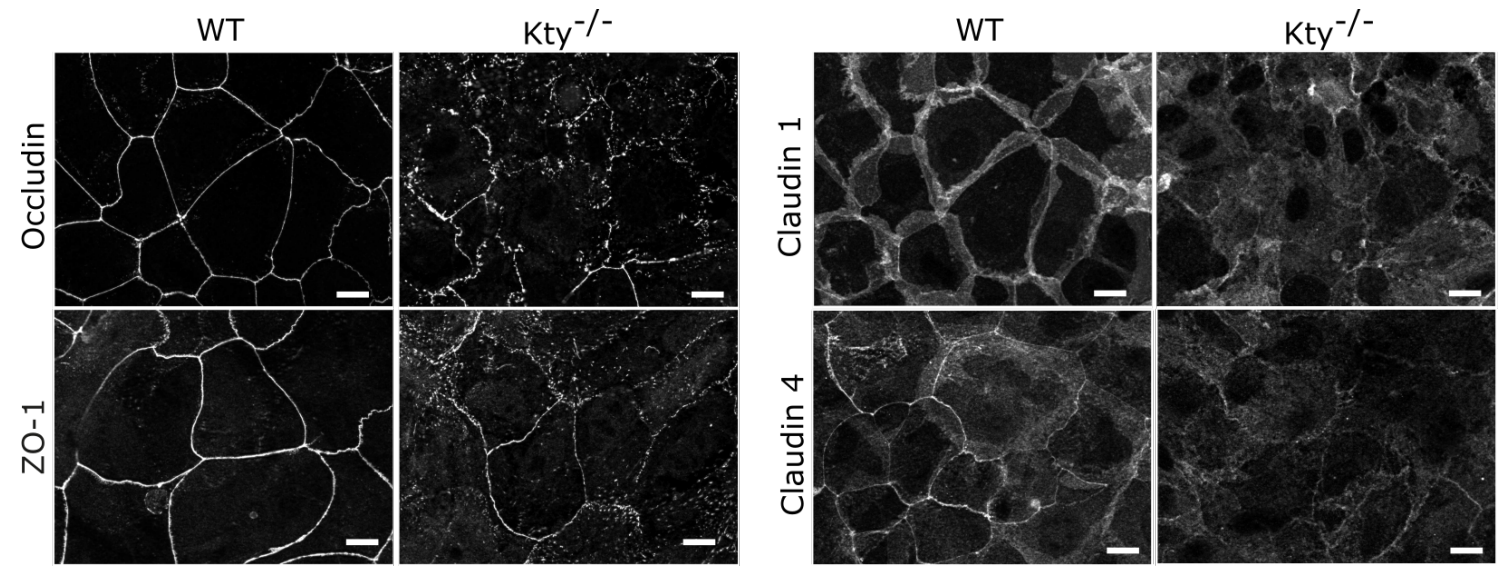

Figure 3.25.: Tight junction protein localization in keratinocyte cells. Fluorescence micrographs showing the localization of occludin, ZO-1, claudin 1 and claudin 4 in wild-type (WT) and knock out (Kty ${ }^{-/-}$) cells. Scale bars: $10 \mu \mathrm{m}$. Experiments and image acquisition were performed by Fanny Büchau.
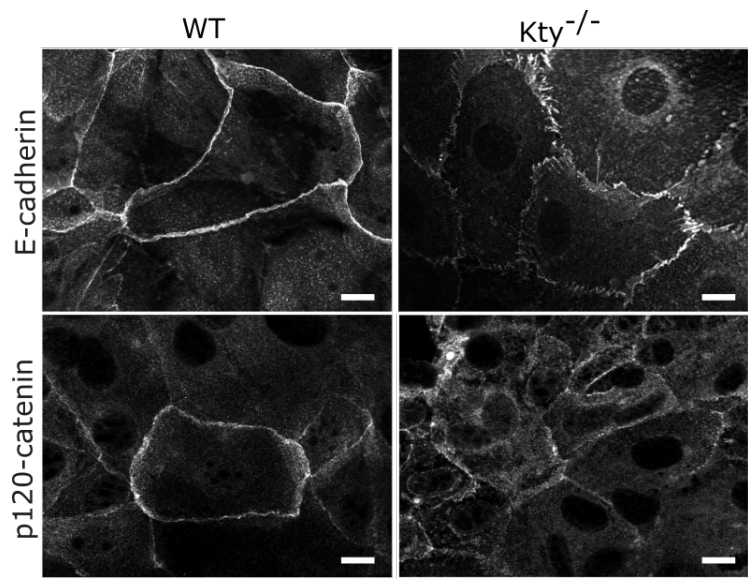

Figure 3.26.: Adherens junction protein localization in keratinocyte cells. Fluorescence micrographs showing the localization of E-cadherin and p120-catenin in wild-type (WT) and knock out (Kty ${ }^{-/}$) cells. Scale bars: $10 \mu \mathrm{m}$. Experiments and image acquisition were performed by Fanny Büchau.

In WT cells the topography of junctions was continuous in height while in $\mathrm{Kty}^{-/}$- cells distinct elevated structures occurred where neighboring cells were in contact with each other. Also gaps between neighboring cell membranes were visible which is in accordance with the optical microscopy observations. Deeper structures across the cell body underneath the membrane were probed simultaneously (figure $3.27 \mathrm{~A}$, zooms). In $\mathrm{Kty}^{-/}$ cells fibrous structures were present which span the cell. This characteristic could not be detected in WT cells which showed a homogeneous height profile also across the cell body. 


\subsection{Influence of keratin deficiency on barrier function and mechanical integrity}

To identify these structures, fluorescence stainings showed that indeed the actin architecture was altered in $\mathrm{Kty}^{-/}$cells (figure $3.27 \mathrm{~B}$ ). While WT cells showed an increased actin signal at the cells' periphery resembling a cortex structure underneath the cell membrane, $\mathrm{Kty}^{-/}$cells revealed distinct actin fibers which spanned the entire cell. Concerning microtubules, no obvious difference in spatial organization of $\alpha$-tubulin was found (figure 3.27 B).

A

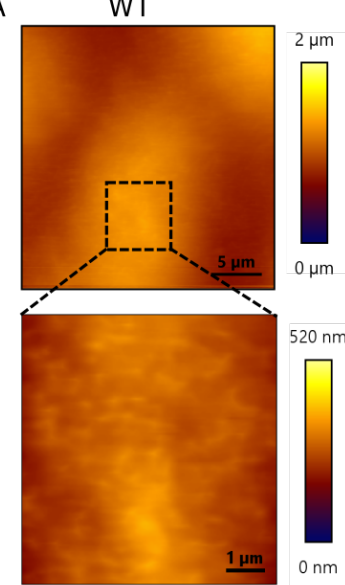

$\mathrm{Kty}^{-/-}$

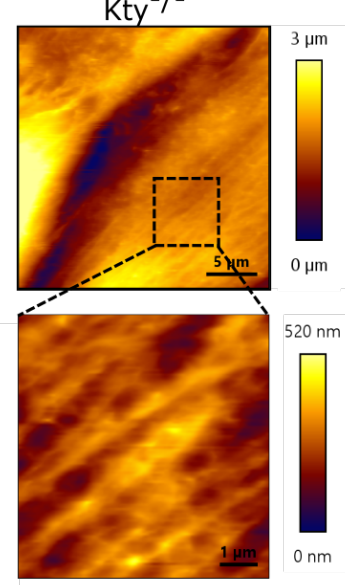

B
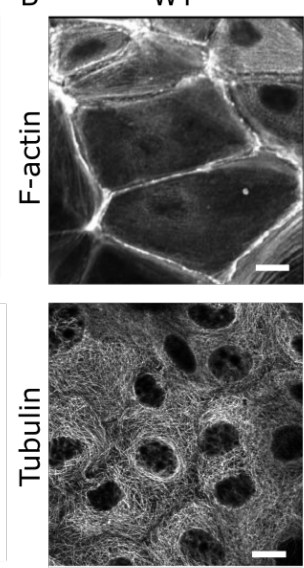

$\mathrm{Kty}^{-/-}$
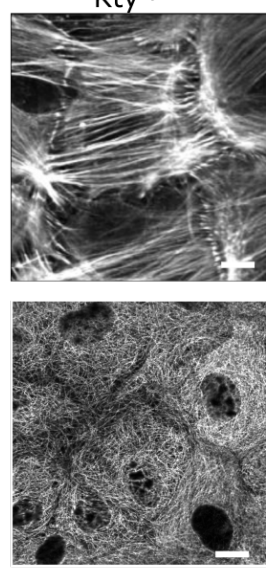

Figure 3.27.: Impaired junctions and fibrous structures in $\mathrm{Kty}^{-/-}$cells. (A) AFM height scans reveal an inhomogeneous junction topography in $\mathrm{Kty}^{-/-}$cells while WT cells show junctions which are continuous in height. Also fibrous structures underneath the membrane of $\mathrm{Kty}^{-/}$cells becomes visible, which are not present in WT cells. (B) Fluoresecence micrographs showing the actin reorganization into thick fibers in $\mathrm{Kty}^{-/}$cells explaining the structures detected with the AFM. In the microtubule network (staining for $\alpha$-tubulin), no obvious change is visible. Scale bars: $10 \mu \mathrm{m}$. (B) Experiments and image acquisition were performed by Fanny Büchau.

Moreover, myosin localization was investigated. For all cells, it mainly co-localized with actin structures (figure $3.28 \mathrm{~A}$ ). In WT cells, myosin was homogeneously distributed at the cells periphery. In $\mathrm{Kty}^{-/}$cells, the distribution was inhomogeneous and signals at junctions were only barely visible in fluorescence micrographs. When analyzing the distribution of active, phosphorylated myosin, the discrepancies between WT and $\mathrm{Kty}^{-/}$ cells were even more pronounced (figure $3.28 \mathrm{~B}$ ). Only a spot-like distribution of phosphorylated myosin at cell-cell junction position were detected for $\mathrm{Kty}^{-/}$cells, while in WT cells the phosphorylated myosin was localized homogeneously across the cells' periphery. In summary, keratinocytes lacking the IF network reorganized actin into thick contractile stress fibers traversing the cell body and showed a mislocalization also of the 
A
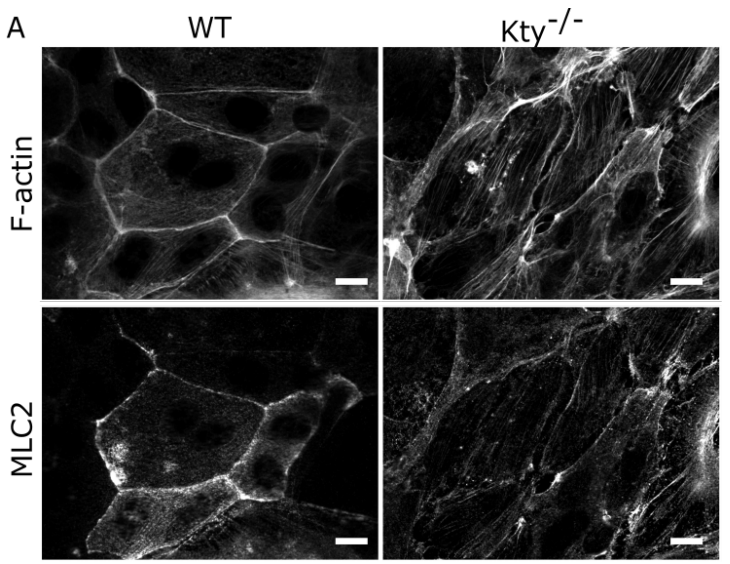

B
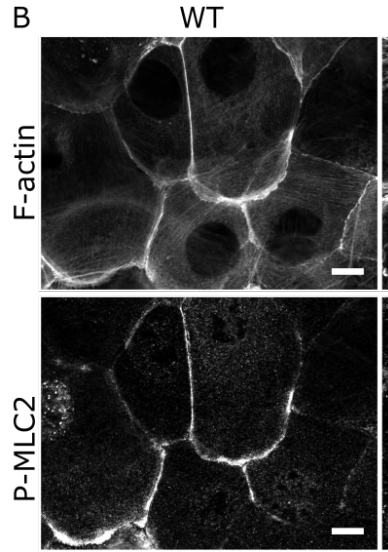
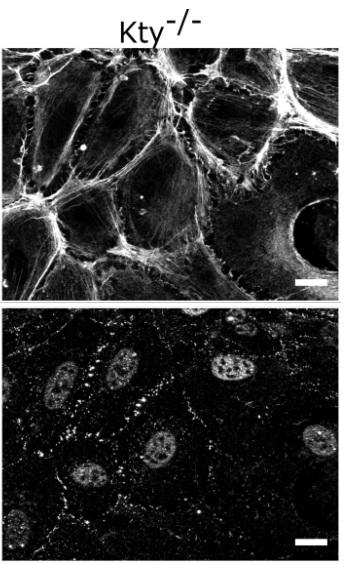

Figure 3.28.: Fluorescence images of myosin localization in keratinocyte cells. (A) Keratinocytes costained for actin and myosin light chain 2 (MLC2) revealing a homogeneous distribution at junctions only in WT cells but not in $\mathrm{Kty}^{-/}$cells. (B) Co-staining of actin and phosphorylated myosin light chain 2 (P-MLC2) shows a strong mislocalization in $\mathrm{Kty}^{-/}$cells compared to WT cells. Scale bars: $10 \mu \mathrm{m}$. Experiments and image acquisition were performed by Fanny Büchau.

actin linked cell-cell junctions comprising tight junction and adherens junctions. Hence, we hypothesized that with the loss of keratin network and the emergence of this new phenotype, the characteristics of barrier maintenance and mechanical integrity could not be maintained within the tissue. To test this hypothesis, we performed functional assays to probe the integrity of barriers against the environment.

\subsubsection{Barrier function is impaired in keratin lacking epidermal cell sheets}

With electric cell-substrate impedance sensing we tested the barrier efficiency of the cell sheet [175]. This method allows to judge the sealing efficiency of the junctions by applying an alternating current and monitoring the resulting complex impedance $Z$ due to current flow through the paracellular space or the membrane. The real part of the impedance at an in intermediate frequency regime of $200 \mathrm{~Hz}<\mathrm{f}<5 \mathrm{kHz}$ is indicative for sealing of the paracellular space by junctions [194] and was evaluated over time. Three different phases were identified (figure 3.29 A). Before calcium addition, both WT and $\mathrm{Kty}^{-/}$cells generated the same low impedance (Phase I named P I) indicative of premature, leaky cell-cell contacts. Residual impedance originated from cells adhering to the gold electrodes. Instantaneously after calcium addition, a barrier to ion flux started to 

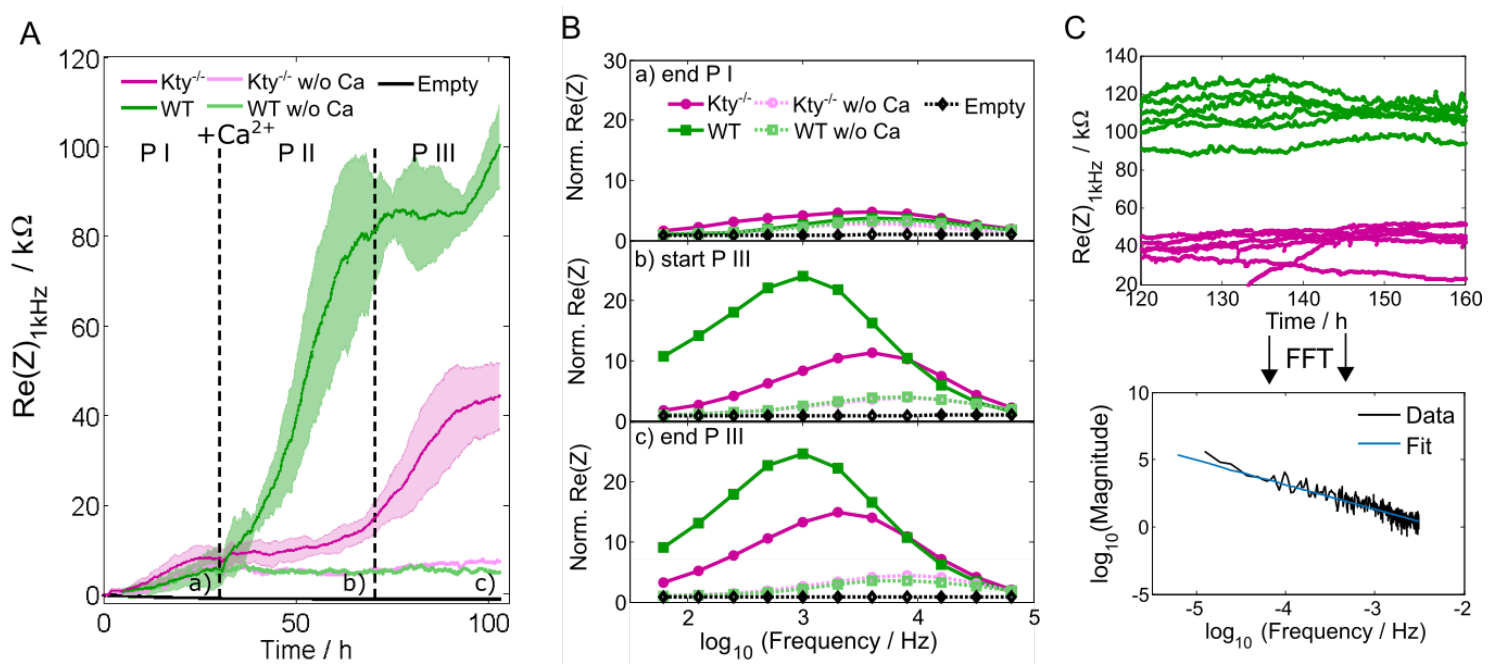

Figure 3.29.: Electrical cell-substrate impedance sensing (ECIS) measurements reveal a barrier malfunction due to junction failure upon keratin deficiency. A) Real part of the impedance $Z$ recorded at $1 \mathrm{kHz}$ as an indicator for junction functionality followed over time for WT (green, $\mathrm{N}=4$ ), $\mathrm{Kty}^{-/}$(magenta, $\mathrm{N}=4$ ), 'no calcium' controls (light green, light magenta, respectively) and empty electrodes (black). B) Normalized impedance spectra a) directly before calcium addition, b) at start of PIII (start of steady state in WT cells) and c) at end of PIII (start of steady state in $\mathrm{Kty}^{-/}$- cells) as indicated in A. C) For fluctuation analysis each individual signal is Fourier transformed (black) and the resulting power spectrum is fitted with a linear slope (blue).

form in WT cells as inferred from an increase in impedance in P II. At the same time, $\mathrm{Kty}^{-/}$cells remained on the impedance values corresponding to those of 'no calcium' controls, indicating that no barrier has formed and junction sealing is incomplete for these cells. Only when WT cells already reached a stable barrier at P III also $\mathrm{Kty}^{-/}$cells started to establish a barrier. Upon impedance saturation, lower values were still found for $\mathrm{Kty}^{-/}$cells in comparison to those of WT cells. Differences in impedance increase upon layer formation can be seen for all intermediate frequencies (figure 3.29 B). As the high frequency regime is mostly dominated by surface coverage and not influenced by junction formation [194, differences between WT and $\mathrm{Kty}^{-/-}$vanished, therefore indicating that indeed junction formation is the reason for differences in impedance levels between WT and $\mathrm{Kty}^{-/}$- cells.

Furthermore, a deeper analysis of impedance fluctuations under steady-state conditions was performed as the signal showed larger fluctuations for WT cells than for $\mathrm{Kty}^{-/}$cells $^{-}$ (figure 3.29 C). Not only is the variance larger but also the memory of impedance fluctuations is different. The resulting power law dependence $\propto \mathrm{f}^{-\beta}$ in the power spectrum 


\section{Results}

with $\beta$ being generally between 0.6 and 2.5 is indicative for the presence of a long time memory effect in the electrical signal [195]; it basically represents the Hurst coefficient $\mathrm{H}$ of the noise $(\mathrm{H}=(\beta-1) / 2)$. A value $\mathrm{H}$ in the range $0.5-1$ indicates a time series with long-term positive autocorrelation. This means that a high value in the impedance time series will probably be followed by another high value and that the expectation for the future will also tend to be that higher impedance values occur. Values larger than $\beta>2$ are indicative of fractional Brownian motion. Indeed, the slope in the power spectrum of WT cells revealed a slope of $\beta_{\mathrm{WT}}=(2.02 \pm 0.18)(\mathrm{N}=12)$. This magnitude in slope indicateed that the fluctuations underlie a memory effect and active noise might be present. In $\mathrm{Kty}^{-/}$cells the slope decreased to $\beta_{\mathrm{Kty}^{-/}}=(1.75 \pm 0.18)(\mathrm{N}=12)$. In earlier studies values of around 2 and larger can be found for living cells and different cell lines while $\beta$ for the same cells fixed with glutaraldehyde were found to be around values of less than 1 [195]. This pointed to active contributions at junction regulation in WT cells while $\mathrm{Kty}^{-/}$cells showed substantially less activity.

Summing up, also functional analysis of the junctions, in particular the tight junctions, showed that the barrier function is impaired in $\mathrm{Kty}^{-/}$cells.

\subsubsection{Altered single cell mechanics and inefficient wound closure in keratin lacking cells}

Next, we investigated the mechanical properties of cells in order to find out whether the change in cytoskeletal architecture mirrored the way cells respond to mechanical stimuli and to what extent mechanical integrity was compromised in knock out cells. As keratinocytes are major players in wound healing, we probed these cells with single-cell wounds. Confluent cell sheets were created by culturing them for $48 \mathrm{~h}$ in high calcium medium before cortices of single cells were scratched with a micropipette leading to a single-cell wound. Success of the treatment could be inferred from the actin signal in the harmed cells which was dramatically reduced, indicating a loss of actin structures due to mechanical destruction of the filaments (figure $3.30 \mathrm{~A}$ ). In WT cells a keratin network with only a hole where the micropipette was inserted was still visible, which showed that the keratin network was able to bear and endure this harsh mechanical treatment while the actin structures already broke down.

The single-wound assay revealed that defect WT cells retained their shape after woun- 


\subsection{Influence of keratin deficiency on barrier function and mechanical integrity}
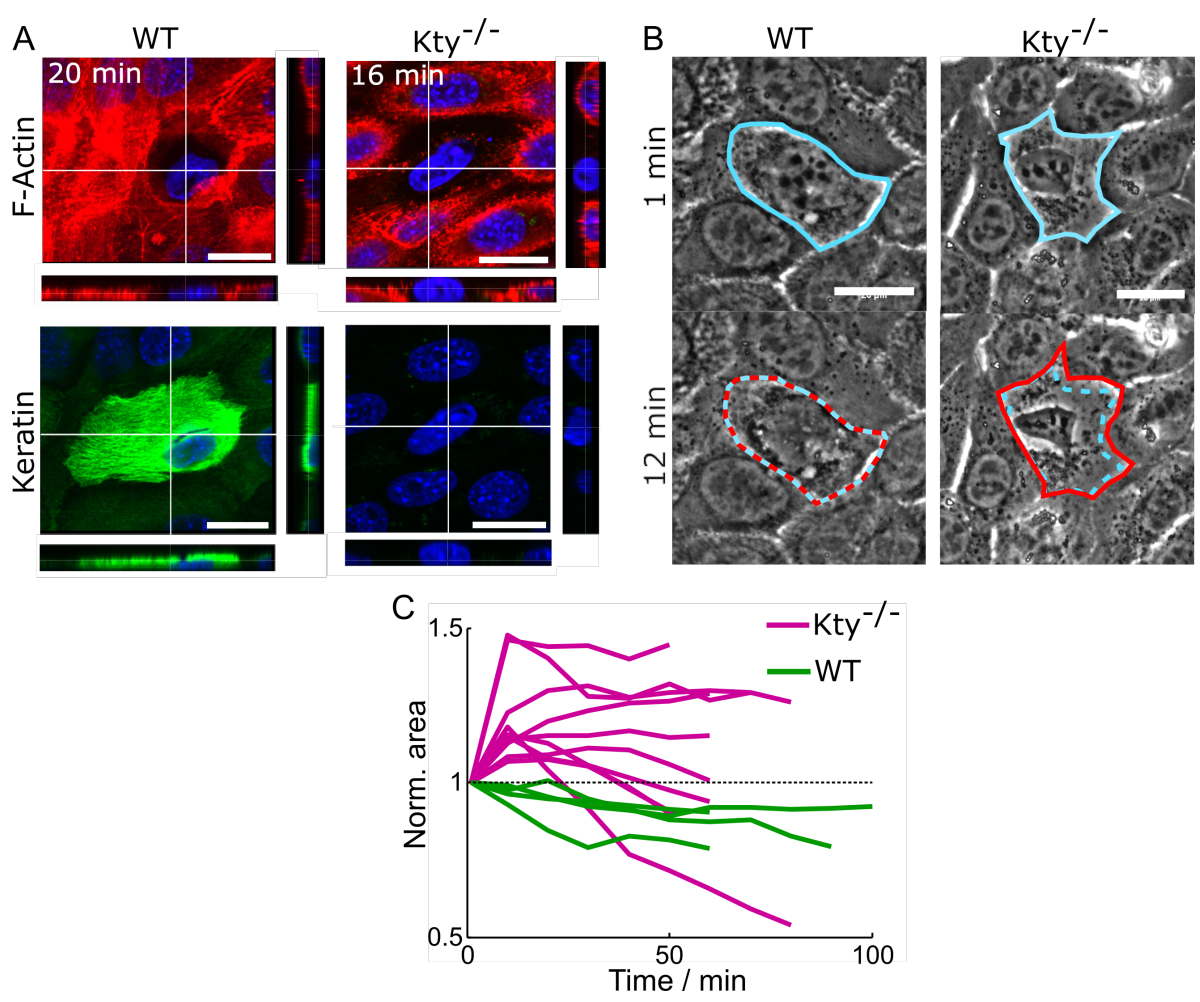

Figure 3.30.: Micropipette wounding reveals the effects of the changed cytoskeletal architecture. (A) Fluorescence micrographs including $x y$ and $x z$ projections of actin (red) and keratin 14 (green) in the wounded cell roughly $20 \mathrm{~min}$ after wounding. (B) Phase contrast images with indicated cell outline $1 \mathrm{~min}$ after wounding (blue) and $12 \mathrm{~min}$ after wounding (red). (C) Individual cell area progression of defect cells over time for WT (green) and $\mathrm{Kty}^{-/-}$cells (magenta). Normalization was done to the area shortly before wounding. Scale bars $20 \mu \mathrm{m}$.

ding (figure $3.30 \mathrm{~B}$ ). In contrast, in $\mathrm{Kty}^{-/}$- cells the cell body opened and fragments got pulled outwards. Also, massive changes in the nucleus appearance became visible. Detailed size analysis confirmed that the wounded region increased in size in $\mathrm{Kty}^{-/}$cells within the first 15 minutes after wounding (figure $3.30 \mathrm{C}$ ) while, in contrast, WT cells started to close the wound by slowly decreasing the area of the wounded cell. This behavior might point to a different relaxation process occurring in $\mathrm{Kty}^{-/}$cells neighboring the wound early after wounding, compared to the situation in WT cell layers.

Concerning the overall cell mechanics, it was found previously that cells lacking the keratin network are softer compared to WT cells [72, 113]. However, these were either AFM measurements on individual and isolated cells or optical stretcher measurements with round cells in suspension. In this study, the tension model was applied to AFM 


\section{Results}

force indentation experiments [8] which were performed on cells grown to confluency where junctional complexes were built between cells being important for cortical tension generation [99]. Indeed, we found that the apparent pretension dominated by cortical tension was decreased in $\mathrm{Kty}^{-/}$- cells (figure 3.31 A) which agreed with the aforementioned results. The apparent area compressibility modulus dominating large indentation depths, especially with sharp indenters, was increased in Kty ${ }^{-/}$cells (figure 3.31 B). Probably this was due to the thick actin fibers crossing the cells, which might also be probed during indentation at large strain.
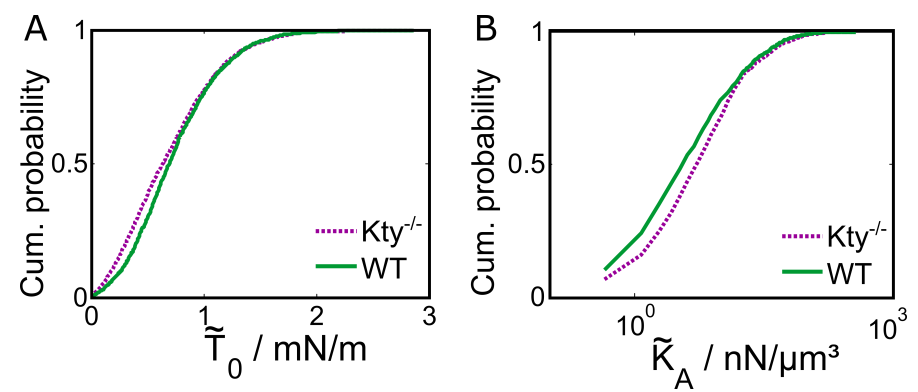

Figure 3.31.: Comparison of cell mechanics according to the tension model for WT (green, solid line) and $\mathrm{Kty}^{-/-}$cells (magenta, dashed line). (A) Cumulative histogram of the apparent pretension $\tilde{\mathrm{T}}_{0}$ capturing the resistance to external force at low indentation depth $(p<0.001)$. (B) Cumulative histogram of the apparent area compressibility modulus $\tilde{\mathrm{K}}_{A}$ probing larger indentation depths $(p<0.001) . n=2441$ for $\mathrm{Kty}^{-/}$cells and $\mathrm{n}=2563$ for WT cells.

\subsubsection{Loss of mechanical coupling in keratin lacking epidermal sheets}

As collective mechanical integrity and intact cell coupling between keratinocytes is fundamental for the function of the skin, both issues were addressed in cells lacking the keratin network. Recent studies showed that junctional complexes are not only important for proper fluid barrier but also they are important for mechanical layer integrity and tension homeostasis within the tissue [52]. Evidence is increasing that cell-cell junctions bear loads, transfer forces through the layer [82], and feedback on the architecture of the actomyosin network (see section 1.4).

Upon mechanical stimulus application during wounding, differences in the mechanical coupling efficiency between the cells were analyzed. In $\mathrm{Kty}^{-/-}$cells only movements of the actually scratched cell were visible. In WT cells, movements also in cells and junctions 


\subsection{Influence of keratin deficiency on barrier function and mechanical integrity}

considerably further away were detected during micropipette movement (figure $3.32 \mathrm{~A}$ ) indicating a mechanical coupling in the cell layer during the stimulus. For $\mathrm{Kty}^{-/-}$cells, no movement was detected at junctions one cell separated from the stimulus (figure 3.32 B) suggesting that mechanical coupling was compromised. Also, mechanical responses
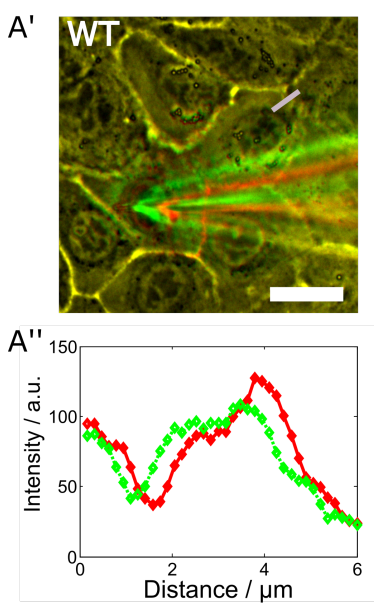
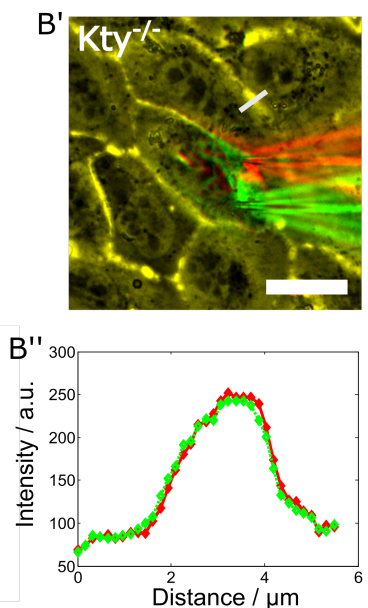
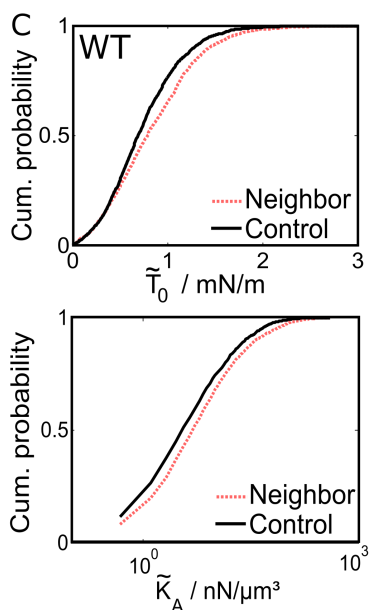
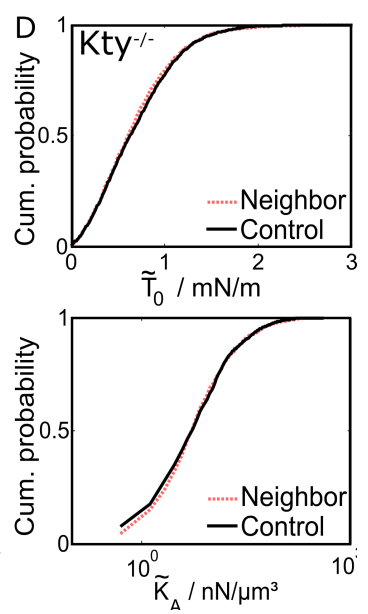

Figure 3.32.: Cells adjacent to the site of single-cell manipulation respond differently to the micropipette wounding depending on the presence of intermediate filaments. (A') Merge of colored (red, green) phase contrast images taken during micropipette movement of WT cells. Yellow color indicating exact matching of structures, red image before micropipette movement, green image after movement. (A") Light intensity line profiles of the two images at a position of a cell junction one cell away from the defect (grey bar in $A^{\prime}$ ) indicating junction movement during wounding. ( $\mathrm{B}^{\prime}$ ) and (B") Same analysis for $\mathrm{Kty}^{-/}$cells during micropipette movement indicating no motion at cell junctions further away from the stimulus. (C) Cumulative histogram of the apparent pretension $\tilde{T}_{0}$ and apparent area compressibility modulus $\tilde{K}_{A}$ of WT cells neighboring a single-cell defect (red) or in an intact cell layer (black) (both $\mathrm{p}<0.001 ; \mathrm{n}=2659$ (neighbor) and $\mathrm{n}=2563$ (control)) (D) Cumulative histograms of the same mechanical parameters for of $\mathrm{Kty}^{-/}$- cells neighboring a defect (red) and control cells in an intact layer (black) $(\mathrm{p}=0.112$ and $\mathrm{p}=0.288$, respectively; $\mathrm{n}=2225$ (neighbor) and $\mathrm{n}=2441$ (control)). Scale bars: $20 \mu \mathrm{m}$.

in cells neighboring a single-cell defect were different for cells possessing an intact keratin network and cells lacking keratin. After defect induction, mechanical changes as response to a neighboring defect were already detected in MDCK II cells (see section 3.4). This was also the case for WT keratinocytes. Changes to larger mechanical resistance became obvious in both parameters, the apparent pretension and the apparent area compressibility modulus (figure $3.32 \mathrm{C}$ ). In $\mathrm{Kty}^{-/}$cells however the same singlecell mechanics were measured in controls cells surrounded by an intact layer and defect neighboring cells (figure 3.32 D) indicating that the cells did not respond to mechanical wounding of neighboring cells. 


\section{Results}

In summary, not only single-cell mechanics was altered but also coupling between cells and hence mechanical tissue integrity was disturbed in cells lacking the keratin network.

\subsubsection{Insertion of $\mathrm{K} 14$ rescues phenotype but not the full functionality}

As seen so far, a complete loss of the keratin network had crucial implications for barrier formation and functionality, single cell architecture, mechanical homeostasis and elastic coupling within the layer. In earlier AFM studies it was shown that a K5/K14 rescue cell line can restore the single-cell mechanics of isolated WT cells [72]. However, in optical stretcher experiments the creep behavior of rescued cells resembled more closely that of $\mathrm{Kty}^{-/}$cells than that of WT cells [113]. Essentially, the question arouse which parts of the phenotype and functionality observed for $\mathrm{Kty}^{-/}$cells can be reversed by reexpression of $\mathrm{K} 5 / \mathrm{K} 14$ to match the properties of wild-type cells. From fluorescence staining, one can conclude that the localization of tight-junction proteins (figure $3.33 \mathrm{~A}$ ) and adherens junction proteins (figure $3.33 \mathrm{~B}$ ) resembled the localization pattern of WT cells. Continuous fluorescence signals at the site of cell-cell junctions were detected indicating proper cell-cell contact. Also, the actin cytoskeleton appeared to be more homogeneous than in $\mathrm{Kty}^{-/}$cells, most probably building a cortex structure at the cells periphery (figure $3.33 \mathrm{C}$ ). The tubulin distribution seemed to be unaffected by the insertion of the keratin network and resembled the distribution found for WT and $\mathrm{Kty}^{-/}$- cells before (figure $3.33 \mathrm{D}$ ). AFM topography scans confirmed that no fibrous structures were present underneath the apical membrane and that the junction topography was not fragmented (figure $3.33 \mathrm{D}$ ). Taken together, the phenotype of junctions and actin of the rescue cells resembled very closely the one of WT cells. Concerning the barrier efficiency measured in electrical cell-substrate impedance measurements, an instantaneous barrier formation was observed in K14 cells after calcium addition as found for WT cells (figure 3.34 A). Nevertheless, also functional discrepancies to the WT behavior were found in ECIS experiments. The impedance at $1 \mathrm{kHz}$ at steady-state fell between the one of WT and $\mathrm{Kty}^{-/}$cells. From complete spectra it was observed that the increase of impedance in $\mathrm{K} 14$ cells for all intermediate frequencies fell in between the WT and $\mathrm{Kty}^{-/}$- cells $48 \mathrm{~h}$ after calcium addition (figure $3.34 \mathrm{~B}$ ). In the high frequency regime discrepancies vanished indicating that only frequencies sensitive to junction formation were affected. 


\subsection{Influence of keratin deficiency on barrier function and mechanical integrity}
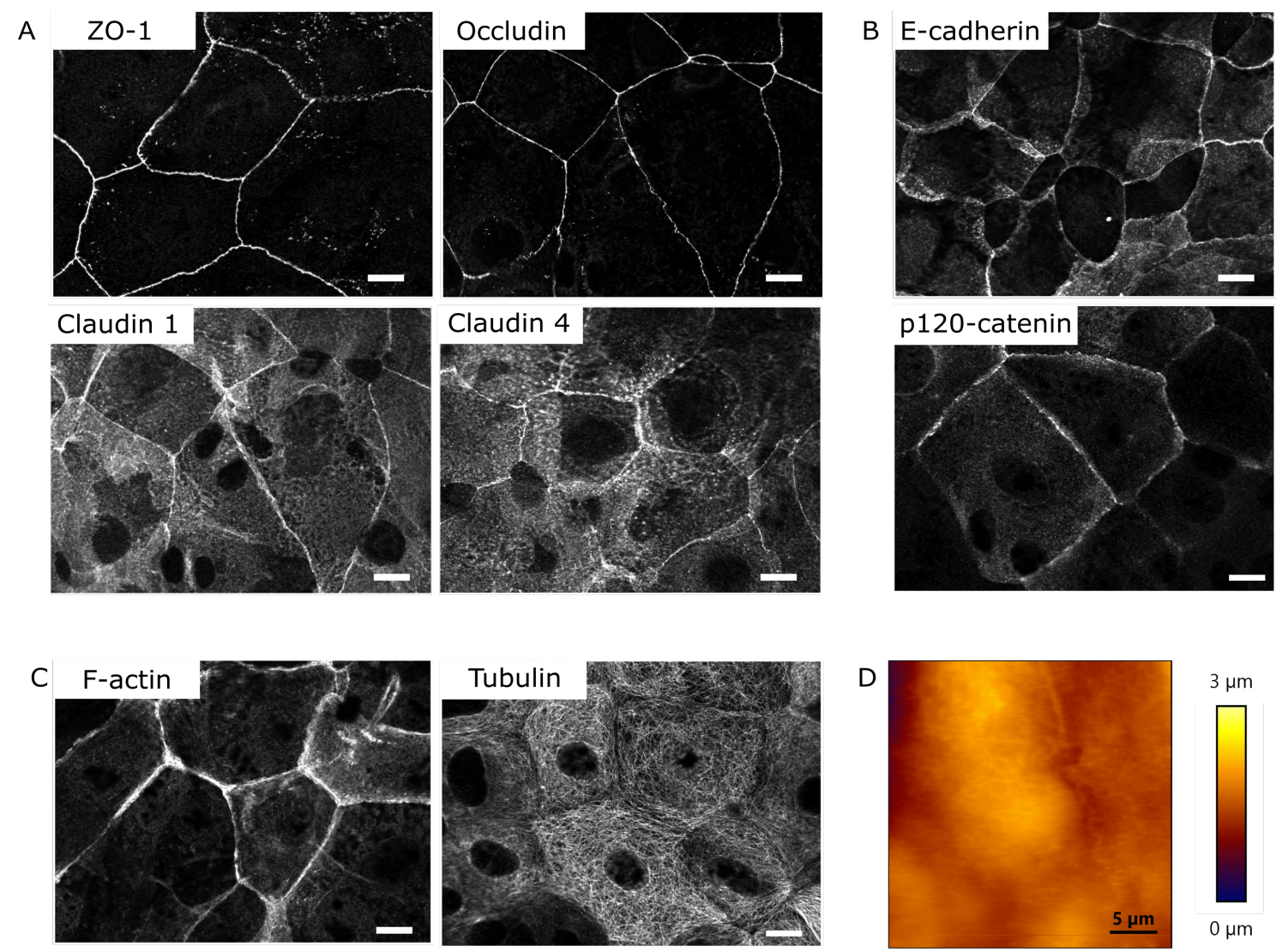

Figure 3.33.: K14 rescue cells show a phenotype which is similar to the one of WT cells. (A) Fluorescence micropraphs showing that due to insertion of keratin 14, the tight junction proteins ZO- 1 , occludin, claudin 1 and claudin 4 relocalize to cell-cell junctions. (B) Also the adherens junction proteins Ecadherin and p120-catenin can mostly be found at cell junction sites. (C) F-actin in K14 rescue cells reorganizes into a cortex at the cells periphery and also $\alpha$-tubulin staining shows a homogeneous network. (D) AFM height scans confirm a continuous junctions topography and homogenous structures underneath the apical membrane, resembling a cortex, as in WT cells. (A-C) Experiments and image acquisition were performed by Fanny Büchau.

Furthermore, fluctuation analysis proved that $\mathrm{K} 14$ cells showed the same level of active noise in impedance measurements as WT cells (with $\beta_{\mathrm{K} 14}=(2.07 \pm 0.20)(\mathrm{N}=12)$ and $\beta_{\mathrm{WT}}=(2.02 \pm 0.18)$, while knock-out cells arrived merely at $\left.\beta_{\mathrm{Kty}^{-/}}=(1.75 \pm 0.18)\right)$. Consequently, the ability to form a functioning barrier was only partially restored.

Moreover, the wound size progression after defect induction mirrored the WT behavior as the wound area is rather constant or even slowly decreasing over time. No relaxation towards larger gaps as in $\mathrm{Kty}^{-/}$cells occured upon wounding (figure $3.34 \mathrm{C}$ ). Also, fluorescence imaging confirmed that the artificially inserted keratin network withstood 


\section{Results}
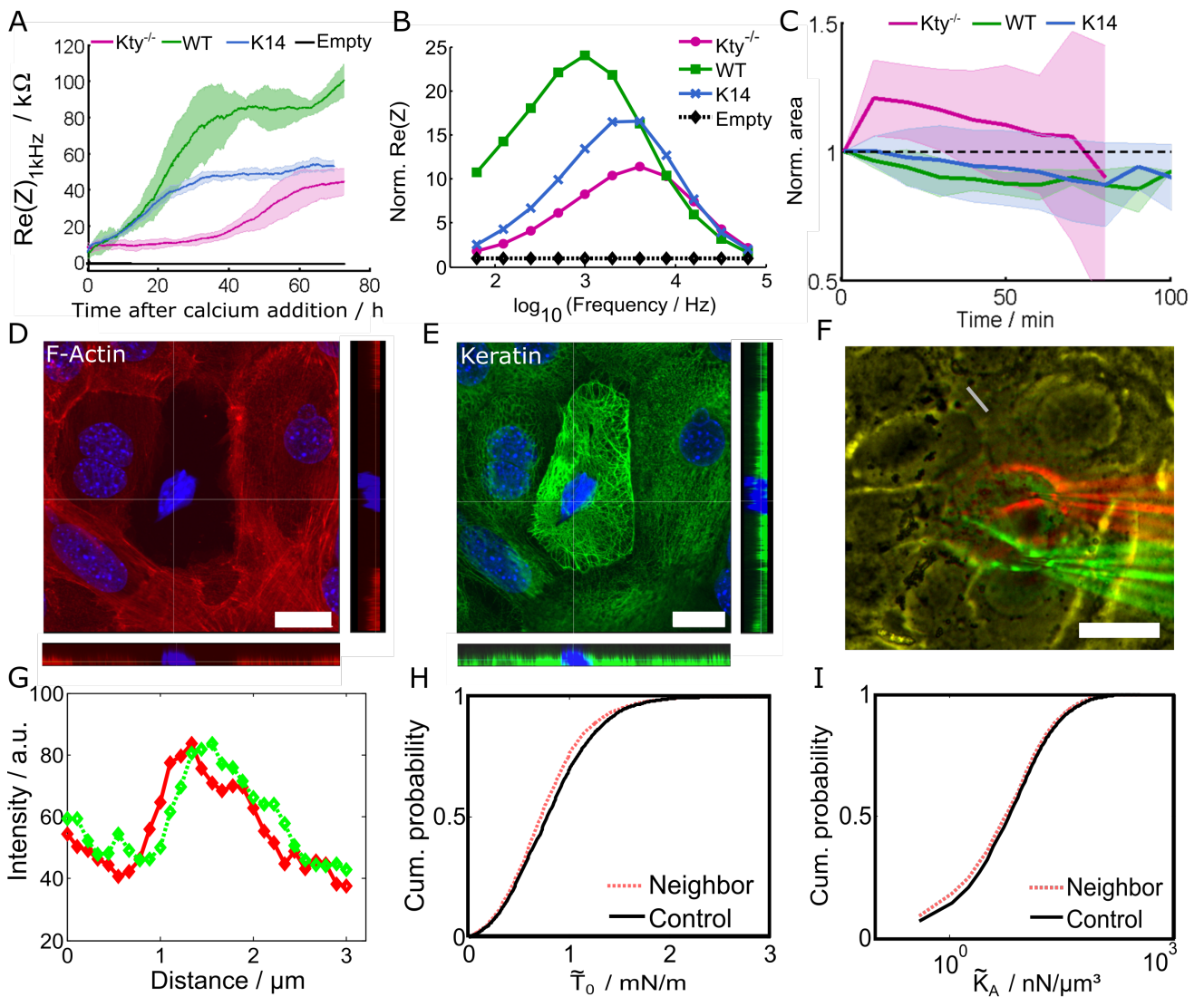

Figure 3.34.: K14 insertion partially restores barrier efficiency and mechanical integrity of cell monolayers. (A) Real part of the impedance $\mathrm{Z}$ recorded at $1 \mathrm{kHz}$ as a representative indicator for junction functionality followed over time for WT (green, $\mathrm{N}=4$ ), $\mathrm{Kty}^{-/-}$(magenta, $\mathrm{N}=4$ ), $\mathrm{K} 14$ rescue cells (blue, $\mathrm{N}=4$ ) and the empty electrode (black). Shown is the average including standard deviation as shaded area. (B) Normalized impedance spectra (real part) $48 \mathrm{~h}$ after calcium addition. (C) Averaged cell area changes of defect cells over time for WT (green, $\mathrm{N}=4$ ), $\mathrm{Kty}^{-/}$- cells (magenta, $\mathrm{N}=9$ ) and $\mathrm{K} 14$ cells (blue, $\mathrm{N}=7$ ) including standard deviations (shaded areas). Normalization was computed with respect to the individual area shortly before wounding. (D) Fluorescence micrographs including $\mathrm{xz}$ and $\mathrm{yz}$ sections of actin (red) and (E) keratin 14 (green) after micropipette treatment. (F) Merge of two colored (red, green) phase contrast images taken during micropipette movement in K14 cells. Yellow color indicating exact overlay of the two images (red before micropipette movement, green after movement). (G) Light intensity profiles of the two images at the position of a cell junction one cell away from the defect (grey bar in $(F)$ ) indicating displacement of junctions during wounding. $(H, I)$ AFM-based mechanical analysis yields the apparent pretension $\tilde{T}_{0}$ and the apparent area compressibilty modulus $\tilde{\mathrm{K}}_{\mathrm{A}}$ of $\mathrm{K} 14$ cells neighboring a defect (red, dashed) and control cells in an intact cell layer (black, solid). Both $\mathrm{p}<0.001$, with $\mathrm{n}=3932$ and 4368 for neighbor and control, respectively. Scale bars: $20 \mu \mathrm{m}$.

the harsh mechanical treatment while actin got disrupted (figure $3.34 \mathrm{D}, \mathrm{E}$ ).

Furthermore, the mechanical coupling in a K14 cell layer was not completely restored 
compared to the wild-type cells. During micropipette movement, junction movement in cells further away was detected (figure $3.33 \mathrm{~F}, \mathrm{G}$ ). However, an increase in stiffness of neighboring cells as a response to wounding as reported for WT cells was not identified in $\mathrm{K} 14$ cells (figure $3.33 \mathrm{H}, \mathrm{I}$ ).

In conclusion, junction and cytoskeleton functionality could partially be rescued with the reexpression of K5/K14 in keratinocytes originally lacking a keratin network. The rescued cells were not fully capable to reestablish an efficient barrier and reach the same level of coupling as WT cells. The remaining discrepancies might be explained by the lack of other keratin isoforms or the lower amount of keratin 5/14 in rescue cells compared to WT cells. 



\section{Discussion}

\subsection{Importance of culture conditions on cell morphology}

The cells morphological changes due to different culture conditions were optically characterized (section 3.1). We found abnormal round structures inside the cytoplasm when MDCK II cells were cultured in medium supplemented with amphotericin B (section 3.1.1). Amphotericin B is used in cell culture experiments without sterile conditions as it is an antifungal agent [196]. As antifungal agent, it is also used in the clinical context in patient treatment against fungal infections [197]. However, studies showed that it has toxic effects in kidney cells of patients [196]. Studies in cell culture systems demonstrated that amphotericin B disturbs normal ion channel function in MDCK cells [197] supporting the findings in patient treatment. Especially interactions of amphotericin B with the plasma membrane are main cause for the associated side-effects [197]. Consequently, it is suggested to avoid the use of amphotericin $B$, especially in experiments with the MDCK cell line, which origins from dog kidney.

Furthermore, phase contrast microscopy imaging was used to document changes in junction appearance of MDCK II cells (section 3.1.1). These changes happened when the culture medium (according to section 2.1) was not replaced frequently during layer growth (roughly every 2-3 days, depending on cell densities). It was observed that replacing the old medium by fresh medium rescued the normal cell morphology. This led to the conclusion that either the consumption of essential medium supplements or an increase in metabolites secreted by the cells most likely caused this problem.

In general, caution should be taken when selecting cell culture medium and supplements, as it is the most important factor in cell culture [198]. Ingredients can decompose, which is the case for glutamine [198], or become toxic upon exposure to light. Light exposure 


\section{Discussion}

in general is a critical factor. In our case it led to morphological changes in MDCK II cells (section 3.1.2). Standard medium ingredients as riboflavin and tryptophan are known to have phototoxic effects upon light exposure [180]. Moreover, studies exist for HEPES, a common supplement for experiments without $\mathrm{CO}_{2}$ supply, reporting that cytotoxic effects may occur [198]. Therefore, exposure to light should be kept to a minimum.

These results demonstrate that during live cell experiments with MDCK II cells, a detailed observation of the cells' appearance in phase contrast microscopy can already give insights into the cells' state. It is likely that the cells' biochemical and mechanical state is affected by the described toxic effects, which might result in uncontrolled and nonreproducible results when analyzing collective behavior.

In addition, the effect of temperature to protein distribution and cell mechanics were analyzed (section 3.1.3). It was found that at room temperature and $43^{\circ} \mathrm{C}$, actin stress fibers are barley present. At intermediate temperatures $\left(30^{\circ} \mathrm{C}\right.$ and $\left.37^{\circ} \mathrm{C}\right)$ the largest amount of stress fibers was detected. Earlier studies found that the extent of actin polymerisation depends on temperature [199]. The extent of polimerization is low at room temperature, increases to a maximum located at $42{ }^{\circ} \mathrm{C}$ and decreases again. This could explain the low amount of stress fibers we found at room temperature. At higher temperatures, protein denaturation might happen. For the actin cross-linking proteins $\alpha$-actinin and myosin denaturation indeed starts around $44{ }^{\circ} \mathrm{C}[200,201]$ which fits to our microscopy observation at high temperatures.

Temperature also affected the cells' mechanical properties (section 3.1.3). Especially the tether force was highly susceptible to temperature changes and decreased with increasing temperature. As tether force can be correlated to membrane tension, which is build up mostly due to membrane cytoskeleton attachments [8], the linker protein ezrin was investigated [24]. No obvious changes in the localization of the membrane linker ezrin were found. Simultaneously, a decrease of the area compressibility modulus and also a decrease in the apparent pretension was found for increasing temperatures between room temperature and $37^{\circ} \mathrm{C}$.

For the three mechanical parameters which were extracted with the tension model from AFM experiments, we found good agreements with the literature:

For the area compressibility modulus, this tendency upon temperature increase (between $5{ }^{\circ} \mathrm{C}$ and $50{ }^{\circ} \mathrm{C}$ ) is in accordance with results from micropipette aspiration experiments 
with red blood cells [202]. Also for the cells' pretension, a decrease for increasing temperature (between $22{ }^{\circ} \mathrm{C}$ and $37^{\circ} \mathrm{C}$ ) was found in neutrophils [203]. However, both values were determined with different cells which were in suspension and with the micropipette aspiration technique. AFM experiments on monocytic THP-1 cells confirmed decreasing tether forces with increasing temperatures (between $16{ }^{\circ} \mathrm{C}$ and $37^{\circ} \mathrm{C}$ ) [204], coinciding with our results.

But in other studies using other parameters to describe cell mechanics, deviating tendencies can be found: Sunyer et al. stated that upon higher temperatures (ranging from $13{ }^{\circ} \mathrm{C}$ to $37^{\circ} \mathrm{C}$ ), human alveolar epithelial cells become stiffer and more solid-like in rheological experiments conducted with an AFM [205]. Kießling et al. found that in optical stretcher experiments, MCF-10A breast cells at $37^{\circ} \mathrm{C}$ are more elastic but at the same time also more fluid than cells at $25^{\circ} \mathrm{C}$ or $15^{\circ} \mathrm{C}$ [206].

Different effects at the molecular level might be temperature sensitive and could contribute to this diverse behavior: Studies which find an increase in stiffness for larger temperatures attribute this behavior to increased actomyosin activity [205, 206]. At the same time, protein unbinding rates increase with in increasing temperature, which was measured e.g. for cytoskeletal protein domains [205] or also the actin-ezrin-membrane complex [207]. Especially the binding of actin to the membrane is a major contributor to membrane tension and subsequently the apparent pretension, which we measured in low indentation regimes with the AFM [8]. Also, a higher membrane fluidity is expected for larger temperatures due to the increased thermal motion of lipids. The relative amount of these effects might depend on the specific cell type, but also on the time scales on which the cells mechanical properties are probed with the specific techniques. For our cells and setup, we found an overall decrease in all extracted mechanical parameters with increasing temperatures.

Only at $25^{\circ} \mathrm{C}$, the area compressibility modulus and the pretension exhibited an irregularity in the trend. The apparent pretension was lower than values at high temperatures and the apparent area compressibilty modulus was the highest measured value. This could be explained with results from earlier studies which showed that at these temperatures a phase transition in the membrane of MDCK II cells happens [208]. So, a drop in pretension and increase in area compressibility modulus seems to accompany this phase transition. 


\subsection{Junction establishment and onset of collective behavior during layer growth}

It was shown that epithelial cells highly rely on contact to adjacent cells. Junctions were established very early and once contact between neighboring cells was established the cells did not separate anymore (section 3.2). Moreover, ZO-1 and E-cadherin localization was monitored during layer growth: While ZO-1 was localized very early at junctions, a significant amount of E-cadherin at junctions was only found at later stages. It is reported from earlier studies that spot-like E-cadherin is required for the early adhesion (see section 1.2.4). Probably these amounts were beyond our detection limit. Still, our results fit to the findings of Gumbiner, who found a large fraction of cytoplasmic E-cadherin [56]. During layer maturation, we found an increase in actin accumulation at the cells' periphery and a change of the cells' height which is in accordance with earlier studies during layer growth [67]. As contractility at junction sites is a major driving force in epithelial cell-cell communication (see section 1.5), actin relocalization to growing cell-cell junctions is a prerequisite to establish such collective behavior.

With the help of substrate patterning, the aim was to force cells in regular contact geometries with defined cell-cell interfaces (section 3.2.3). As single cells adapted to the shape of the adhesive areas on the substrate, it was documented that the patterning method was working well. Also contractile actin fibers arranged according to the pattern. This observation was in accordance with results from earlier patterning studies using single cells [209, 210]. Tseng et al. showed that epithelial cell pairs on patterns (formed like an $\mathrm{H}$ ) position their junction in dependence of the extra cellular matrix protein distribution [211]. With our rectangular geometries, two cells on neighboring adhesive areas formed contact and extended to the adjacent area, leading to a loss of order. Upon layer growth, the loss of order increased and arbitrary positions across the pattern were taken. Recent studies might give an explanation to this behavior: Noethel et al. showed for keratinocytes that vinculin, being present in focal adhesions and adherens junctions simultaneously, might work as a switch between dominant behavior of one of these adhesion types [90]. They stated, that upon formation of a monolayer, a mechanosensitive transition from focal adhesions as major signaling hubs to adherens junctions happens and that this transition is mediated by vinculin. This could explain our observation that once neighbor contact is established, the guidance by the adhesive 
patches of the substrate gets lost.

Moreover, we found that cell clusters can occupy large non-adhesive areas. For other types of epithelial cells it was shown that they can build free standing bridges without any substrate contact underneath [124]. Even though they showed that MDCK II cells were not able to build these free standing bridges, we demonstrated that MDCK II cells can form confluent layers over non-adhesive areas.

This approach demonstrated that cells embedded in a layer and having cell-cell interactions do not adapt efficiently to patterned surfaces and cell-cell interactions dominate the behavior, instead of adhesions to the substrate.

\subsection{Classical large-scale wound healing assays}

Different strategies were applied to induce wounds and disturb mechanical layer homeostasis in MDCK II cells. A scratch assay and a culture insert assay were used first as classical large-scale wound healing strategies (section 3.3). In these wound healing assays, the wounds measured more than $100 \mu \mathrm{m}$ in width. Earlier studies found that for these wounds, cell sheet migration takes place and also cells which are not directly adjacent to the wound respond by specific actin assembly, which indicates collective cell behavior [144]. With monolayer stress microscopy it was found that stress distributions at cell-cell junctions are dramatically heterogeneous in migrating MDCK II monolayers [70]. Especially leader cells at the tip of migration fingers are larger [143] and they show a higher contractility compared to other cells at the wound border or follower cells [118]. Additionally, it was found that different wound closure mechanisms exist. Besides migration, also a supracellular actomyosin cable can be used to close wounds [146]. To which extent the two mechanisms are used can depend on the stage of wound closure [151] or also local wound geometry [141].

We found that during closure of wounds created with these essays, the cells' morphologies were very diverse (figure 3.16 A-C). Very elongated cells and even cells being detached from the substrate were found. In case the cells at the wound margin were intact, both closure strategies of purse string and lamellipodia formation for migration were used by the cells (figure $3.16 \mathrm{D}-\mathrm{F}$ ). Concerning mechanical studies it was shown that cell size and geometry can largely influence the cells' mechanical behavior even in a confluent cell layer [164]. Here, the cells are migrating and constantly remodelling over time. This 


\section{Discussion}

leads to very diverse cell morphologies and wound closure strategies as seen in this study. This diversity most presumably overshadows effects of micro-wounding which is defined as loss of neighboring cells according to section 2.2. Therefore, classical wound healing assays were not further taken into account in this study but are still a valuable tool for analyzing large scale collective behaviors such as cell sheet migration.

\subsection{Effects of single-cell defects in MDCK II layers}

Parts of the following section (including figure) are published in the article Single-cell defects cause a long-range mechanical response in a confluent epithelial cell layer [136]. Text is taken either word-by-word or is closely related.

It was found that as response to a micropipette-induced single-cell defect in a MDCK II cell layer, neighboring cells efficiently close the wound by the well-defined purse string mechanism (section 3.4.1). Moreover, as response to wounding, a change in their mechanical properties was measured (section 3.4.2). We suggest that the increase of the apical pretension must be evoked by increased actomyosin contractility in the cell cortex of cells responding to the existence of a neighboring wound. So, not only generation of contractility in the purse string happens during closure but also in the apical cell cortex. It is consensus that contractility at the wound margin is a key feature to realize proper wound closure [153-155]. Also our experiments with the rho kinase inhibitor Y27632 showed that contractility is a prerequisite for proper purse string formation (section 3.4.6). Simultaneously, reinforcement of actomyosin cortex might be due to the coherence of the actomyosin cortex supporting the cells plasma membrane at the cell periphery [212]. As the effect of increased cortex tension was also seen in larger wounds where the purse string is less dominant, it implies that the contractility of the purse string might not be the only factor explaining a higher prestress stored in the apical cell membrane.

Notably, a reduction of cortical tension at cell-cell contacts is required to maintain connectivity and reach homeostasis in a cell layer [52]. This is due to the fact that the energy gain due to cell-cell-adhesion (e.g. by forming molecular contacts such as those established by E-cadherins) is not sufficient to enlarge the interfacial area and generate appreciable adhesion [213]. As cortical tension comprises cell-cell-adhesion [214], it has to be reduced for proper layer integrity [52]. This can also nicely explain the large pre- 
tension found for front cells at the boundary of a subconfluent cell layer (section 3.4.3). Here, the tension is largely increased as no layer homeostasis can be established at this point. Along the same line, disturbing layer integrity by inducing a single-cell wound seems to disturb homeostasis and leads to a shift in the mechanical phenotype towards increased cortical tension. This goes also in line with earlier studies showing the same effect, e.g., for the epithelial-to-mesenchymal transition [188]. There, a mesenchymal cell displays much larger cortical tension compared to an epithelial phenotype.

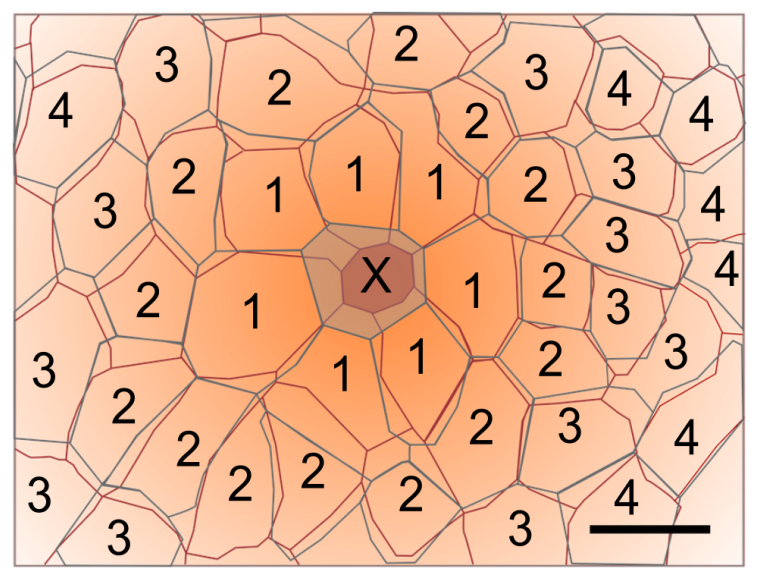

Figure 4.1.: Schematically visualized spatial extension of mechanical changes. A manually skeletonized image shows the affected cells of the single-cell defect, with their assigned shell numbers, before (gray) and $60 \mathrm{~min}$ after (red) wounding of the center cell (X). Scale bar: $20 \mu \mathrm{m}$.

Also, the presented findings suggest that a confluent epithelial cell layer mechanically responds in a collective manner to micro-wounding (section 3.4.4). This mechanical behavior of the unperturbed neighbors points towards a mechanical syncytium rather than an assembly of individual cells (figure 4.1). For larger wounds alterations in cells further away were already reported [125], however, in this study the wound is small, namely on the size of a single cell. How exactly tension is transmitted to cells far away from the defect is still unclear. Both the action of an actomyosin purse string and crawling cells dragging their neighbors towards the wound are conceivable factors to increase the tension in the apical sheet. In any case, cell-cell contacts such as adherens junctions are prime candidates to explain the long-distance transmission of stress: it was shown that long-scale interactions depend on the functionality of cell-cell junctions [121] and apical-lateral junction contractility is fundamental for proper cell layer homeostasis [215]. The force-activated enhancement of cell-cell junctions and cadherin-cytoskeletal inter- 


\section{Discussion}

actions might be a key factor for signal transduction via cell-cell junctions [80, 83, 86]. Experiments from this study where E-cadherin was either fluorescently tagged in living cells or stained in fixed cells showed an increased signal around the defect cell within the first 20 minutes after wounding (section 3.4.6). This shows that a relocalization of E-cadherin indeed happens after single-cell wounding. Mechanical changes, however, are long-lasting and also detectable further away from the wound which can not be explained by the observed E-cadherin appearance. Also, traveling stress patterns inside the layer, mediated by cell-substrate adhesion complexes, seem to be involved in epithelial wound healing on elastic substrates [120, 151]. As both of the adhesion complexes, to the substrate and to neighboring cells, seem to be tightly coupled in a cell layer [216, 217], both adhesion mechanisms might be involved at the same time during wound closure. Moreover, chemical signaling through GTPase activity at cell-cell junctions during wound closure points to a junction mediated coupling between neighbors [218]. Another factor are traveling calcium waves, which were observed after wounding [191, 219] and can induce F-actin reorganization [220] after wounding. We also found calcium responses after single-cell wounding but these were within the first two minutes after wounding and hence took place only at the onset of wound closure (section 3.4.6). The calcium wave was able to travel over several cell widths accounting for the spatial outgrowth of the mechanical response, but the wave alone can not explain the mechanical response in the cells' cortex measured over longer times.

Summing up, these results suggest that the mechanotransduction is relayed over large distances within an intact cell monolayer. Cortical tension might be used by the cell layer as a source of information to prompt a large number of cells helping to close the wound. This far-reaching mechanical effect distributes the burden of single-cell wound closure over many cells.

\subsection{Impact of keratin deficiency on mechanical homeostasis in keratinocyte cell sheets}

Parts of the following section (including figure) are published in the article An intact keratin network is crucial for mechanical integrity and barrier function in keratinocyte cell sheets [192]. Text is taken either word-by-word or is closely related. 


\subsection{Impact of keratin deficiency on mechanical homeostasis in keratinocytes}

The importance of the keratin network for a mechanical homeostasis in epithelial cell layers was demonstrated (section 3.5). It was shown that its absence not only affected desmosomes but also tight junctions and adherens junctions were affected (section 3.5.1). Junctional proteins revealed a relocalization and subsequently a reduced barrier efficiency of the cell layer was measured. Most strikingly, the cells' contractile elements rearranged and thick stress fibers arose that spanned the keratin lacking cells. This change in the individual cell contractility pattern can explain most of the presented findings during and after wounding.

It is known that adherens junctions and tight junctions can be regulated by the actin cytoskeleton [221]: Force positively influences adherens junction size [80] and we saw that junctions in $\mathrm{Kty}^{-/}$cells only form at the tips of the fibers. This leads to an irregular adherens junction-associated protein distribution along the cell-cell junction.

Also for tight junctions, it was shown that actin regulates the barrier efficiency in epithelial cells due to contractile force applied at the junctions [98] which can even influence tight junction positioning within a tissue [59]. Also establishment of adherens junctions and tight junctions is locally coupled (see section 1.2.4). If intracellular applied force at junctions is very localized to distinct spots and consequently irregular positioning of junctions occurs, a loss of barrier functionality as seen in ECIS experiments can be explained (section 3.5.2). Moreover, a loss of memory in the impedance signal points to a less coordinated regulation of junctions over time, which can origin from an impaired actomyosin contractility. Since we found that the noise in the impedance signal generated by the barrier is rather Gaussian and therefore uncorrelated compared to the WT cell layer, absence of concerted action of actively applied forces can be assumed in $\mathrm{Kty}^{-/}$ cells.

A tight regulation of contractile and load bearing elements is crucial for single cell mechanics and also collective cell mechanics. According to the tensegrity model, a composite network of filaments with different mechanical properties build up a flexible but at the same time mechanically stable three-dimensional architecture inside individual cells [103.

We suggest that with the rearrangement of contractile actomyosin inside the cell, the cells try to compensate for the loss of keratin filaments to maintain mechanical homeostasis: keratin filaments prevent the cells from rupture upon large strain, which actin cannot compensate due to different filament mechanics [111]. This was also seen du- 


\section{Discussion}

ring wounding, where mechanical load in WT cells was transmitted to neighboring cells using junctions further away from the actual micropipette movement (section 3.5.4). In $\mathrm{Kty}^{-/}$cells this mechanism is suppressed and only individual cells have to cope with local mechanical disturbance which can result in increasing gap formation at junctions or complete rupture. As these cells are able to spread, grow and form junctions, some kind of mechanical homeostasis necessary for survival is established also in these keratin lacking cells. However, due to wounding with a micropipette and subsequent loss of actin filaments, the contractility in one cell was externally disturbed. Then $\mathrm{Kty}^{-/}$cells were not capable to rely on established procedure for wound closure and the void increased resulting eventually in rupture of the cell layer (section 3.5.3).

In general, it could be demonstrated that epithelial cells in a homogeneous homeostatic layer are in a pretensed state [52] and upon large scale wounding the tissue locally relaxes as indicated by an initial increase in wound size [125]. However, upon single-cell wounding neither MDCK II cells nor WT keratinocytes showed an increase in the gap directly after wounding, as found for $\mathrm{Kty}^{-/}$cells (section 3.5.3). Therefore, we hypothesize that $\mathrm{Kty}^{-/}$cells are even under a larger pretension which is released upon micropipette wounding and loss of contractile fibers.

The idea can be illustrated with a spring model accounting for actomyosin contractility (figure 4.2): In $\mathrm{Kty}^{-/}$- cells, tensed mechanical springs are arranged in series, where one spring symbolizes a single contractile bundle which is connected to a neighboring spring at spots where cell-cell junctions are perpendicular to the cell boundary. If all springs inside one wounded cell $(X)$ fail instantaneously, the springs in neighboring cells recoil to a new resting length and the wound area increases consequently. In WT cells, however, a well-connected homogeneously contracted cortex exists which stabilizes the layer even upon loss of all contractile elements inside one cell. Probably, intermediate filaments act as shock absorbers that slow down initial relaxation upon wounding.

Hence, in WT cells the junctions stayed local upon wounding before a slow closure process started. Here, the neighbors were even able to strengthen their cortex, which was inferred from the increased mechanical pretension in AFM experiments (section 3.5.3). $\mathrm{K} 14$ rescue cells did not exhibit characteristics of either $\mathrm{Kty}^{-/-}$cells or WT cells but often showed an intermediate behavior (section 3.5.5). Junctions and cortex were more homogeneous than in $\mathrm{Kty}^{-/}$- cells and micropipette movements were transmitted throughout the layer. Also, the cells exhibited a reduced contractility compared to $\mathrm{Kty}^{-/}$cells as 


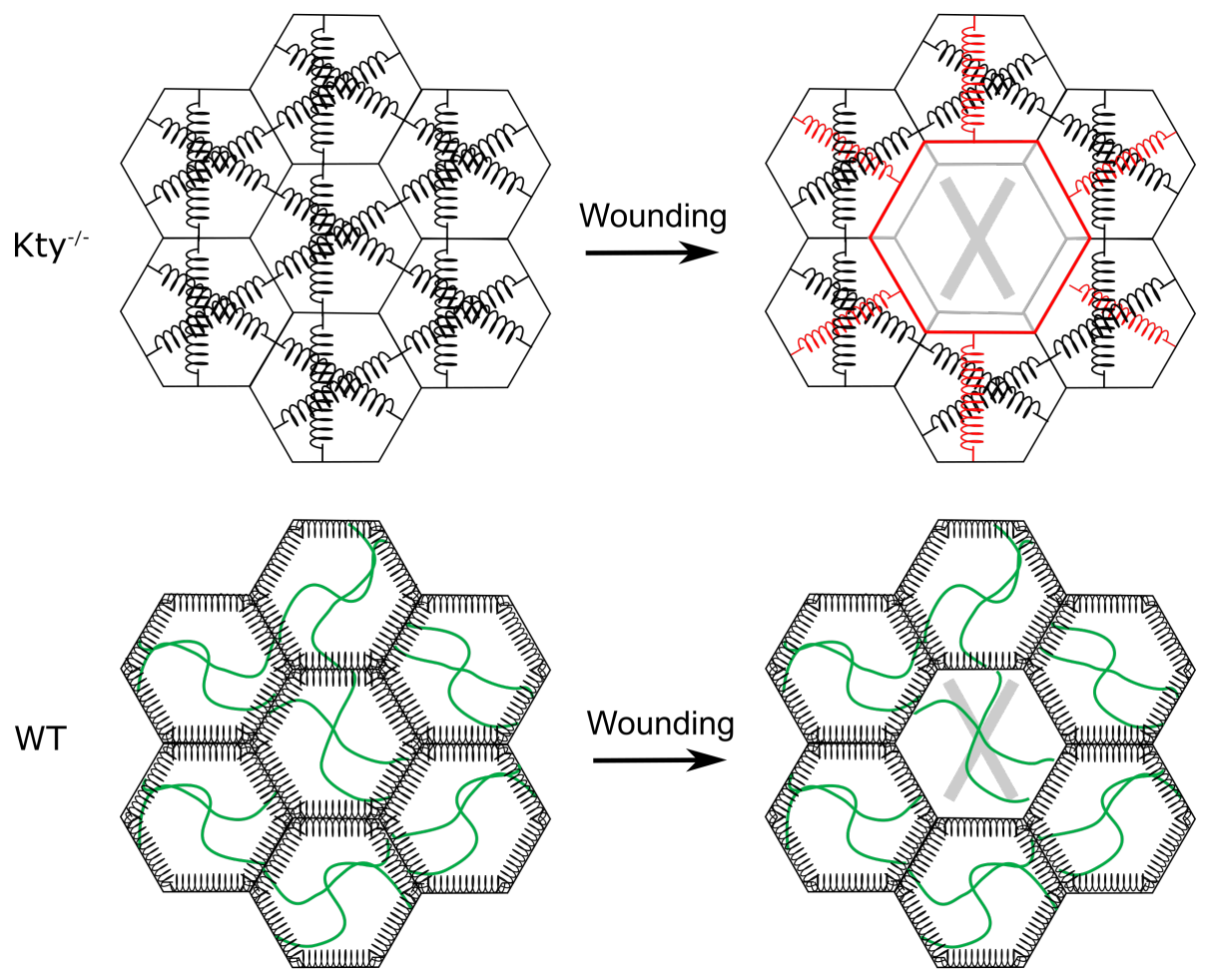

Figure 4.2.: Schematic model of the wound response in $\mathrm{Kty}^{-/-}$cells and WT cells. Actomyosin in $\mathrm{Kty}^{-/-}$ cells is bundled into thick fibers spanning the cell as indicated by springs. Only at the end of the fibers, cells are connected via cell-cell junctions. In WT cells a contractile cortex exists lining the cell. Intermediate filaments (green) are distributed inside the WT cells acting as shock absorbers. Cell-cell junctions with this architecture can be distributed all over the cells' periphery. Upon wounding and hence loss of contractile filaments inside one cell $(\mathrm{X})$, different responses occur: In $\mathrm{Kty}^{-/-}$contractile filaments release tension, shrink to a shorter resting length which leads to a wound increase (red). In WT cells forces are distributed more evenly so that stable junction positions and resting lengths of the contractile filaments are possible.

indicated by the wound size progression. These characteristics more resembled the WT cell behavior. However, tight junction regulation was still altered compared to WT cells as seen by ECIS measurements and the coupling was less pronounced, pointing to persisting discrepancies to WT cells. One reason might be that the keratin pair $\mathrm{K} 5 / \mathrm{K} 14$ is present to a lesser extent in rescue cells than in WT cells [113]. Also, other keratin isoforms are not or less present than in WT cells. So, the overall keratin amount is lower in rescue cells than in WT cells, which can explain the incomplete rescue of functionality. The general hypothesis is that due to lack of keratins, the actin cytoskeletal structure changes to maintain a stable mechanical phenotype by contractile elements spanning the cell. As force positively influences E-cadherin recruitment and subsequently junction 


\section{Discussion}

size [80], this could promote junction formation only at the ends of thick actomyosin fibers. This hypothesis could explain the mislocalization found for junctional proteins leading to impaired cell-cell adhesion. However, also direct mislocalization of junctional complexes and subsequent recruitment of actin to distinct spots at the cells periphery would result in the same phenotype observed here. So, different signaling mechanism might be plausible which lead to this new phenotype.

Still, with this new mechanical phenotype, the cells are vital as also seen in earlier studies [72, 73, 113]. However, this phenotype with increased contractility and fragmented junctions is very fragile. The junctions cannot efficiently seal the monolayer and the mechanical integer behavior of the layer is lost. This highlights the importance of keratin IFs for tissue function and mechanical homeostasis in keratinocyte cell layers. 


\section{Conclusion}

In this study, epithelial cells were characterized during layer formation and critical culture conditions. This allowed to monitor the onset of collective behavior and prerequisites for proper layer function. It was shown that cell-cell junctions dominate the cells behavior from an early stage of layer formation. They mature over time and allow the cell to build a large functioning collective. For live experiments, it was important to characterize the cells behavior upon different culture conditions such as media supplements and temperature. These experiments showed that cells are highly susceptible to these conditions and that they can influence the cells' mechanical properties.

With the new approach of micro-wounding, homeostatic conditions in a confluent cell monolayer were locally destroyed. Monitoring the wound responses in such a reduced in vitro system, allowed to gain a further understanding which contributions are prerequisite also for complex macroscopic wound healing. In MDCK II cells, single-cell wounds on the size of $\approx 20 \mu \mathrm{m}$ in diameter lead to striking effects. Defect neighboring cells which close the wound also exhibit an increased pretension. Even though the perturbation is small, precisely on the size of a single-cell, far-reaching effects can be measured also in several tens of microns away from the defect. This far-reaching signal transduction is most probably mediated by the cells mechanosensitive elements as cell-cell junctions and the cytoskeleton. This study demonstrates that collective behavior is not only restricted to large scale phenomena such as migration but also small disturbances as in micro-wounding elicit a coordinated response.

We hypothesized that tension generation inside the cells and the layer is an important signal triggering the collective response after micro-wounding. This was supported by analyzing the interplay between actomyosin and the intermediate filament network in homeostatic conditions and after micro-wounding in keratinocytes. In keratinocytes artificially lacking the keratin network by gene engineering, a new mechanical phenotype arises to compensate the loss. As a result, a barrier dysfunction and junction mislocal- 


\section{Conclusion}

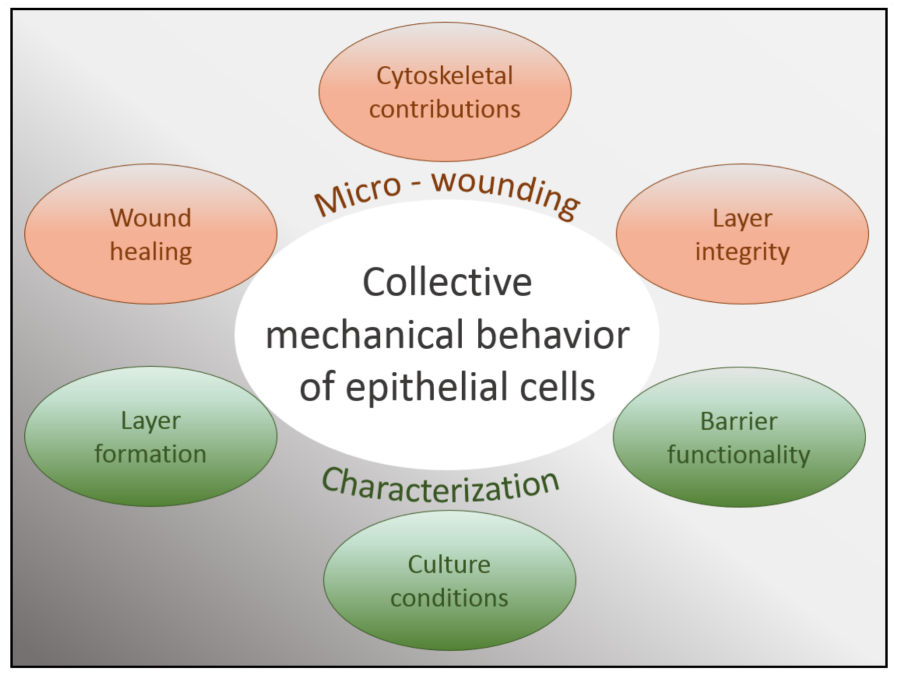

Figure 5.1.: The collective mechanical behavior of epithelial cells was analyzed in this study. Aspects of layer formation, culture conditions and barrier functionality to characterize collective mechanical behavior are investigated. Furthermore, the method of micro-wounding was applied to analyze micro wound healing and the importance of collective behavior for wound closure. This wounding method further allowed to decipher cytoskeletal contributions and the importance of layer integrity and collective behavior in wound closure.

ization occurs. With the micro-wounding technique we showed that these cells loose functional layer integrity which ensures proper wound response in wild-type cells. A mechanism was proposed, which relies on an altered tension distribution within these cells compared to cells containing an intact keratin network. With the tension distribution inside the keratin-lacking cells, layer functionality and the response to wounding gets lost. These findings highlight the importance of the intermediate filament network and subsequently well-controlled single-cell mechanical properties in cell layer homeostasis.

A more general approach for the future would be the use of $3 \mathrm{D}$ cell culture as also epithelial cells in the body build a multilayered system. This approach would go more in direction of the in vivo situation. Three dimensional cell culture systems are more and more advancing and will help to tackle more complex questions of proper cell function in the human body [222, 223]. Most presumably, collective behavior in three dimensions will change because adhesion complexes are build and located differently [224] and stress patterns across cells will change in a three dimensional tissue. Then wounding would become a three dimensional process where several cell layers are affected and have to respond. Moreover, co-culture of different cell lines would be useful to mimic the in 
vivo situation even more closely, as several cell types are involved in macroscopic wound healing in nature.

Adding more complexity to in vitro studies will help to understand collective behavior also in larger structures such as tissues or organs. Still, a lot of research with reduced systems is necessary to finally complete the picture of wound healing and collective phenomena in organs or even the human body. 



\section{Bibliography}

[1] L. Eckert, "Structure, function and differentiation of the keratinocyte," Physiological reviews, vol. 69, no. 4, 1989.

[2] J. Rother, H. Nöding, I. Mey, and A. Janshoff, "Atomic force microscopy-based microrheology reveals significant differences in the viscoelastic response between malign and benign cell lines," Open Biology, vol. 4, no. 5, 2014.

[3] G. C. Gurtner, S. Werner, Y. Barrandon, and M. T. Longaker, "Wound repair and regeneration," Nature, vol. 453, no. 7193, pp. 314-321, 2008.

[4] J. B. Reece, L. A. Urry, M. L. Cain, S. A. Wasserman, P. V. Minorsky, R. B. Jackson, et al., Campbell biology. Pearson Boston, 10 ed., 2014.

[5] B. Alberts, A. Johnson, J. Lewis, M. Raff, K. Roberts, and P. Walter, Molecular biology of the cell. Taylor \& Francis Group, 5 ed., 2007.

[6] O. S. Andersen and R. E. Koeppe, "Bilayer thickness and membrane protein function: an energetic perspective," Annual Review of Biophysics and Biomolecular Structure, vol. 36, no. 1, pp. 107-130, 2007.

[7] J. N. Israelachvili, Intermolecular and surface forces. Intermolecular and Surface Forces, Elsevier Science, 3 ed., 2015.

[8] A. Pietuch, B. R. Brückner, T. Fine, I. Mey, and A. Janshoff, "Elastic properties of cells in the context of confluent cell monolayers: impact of tension and surface area regulation," Soft Matter, vol. 9, pp. 11490-11502, 2013.

[9] D. Raucher and M. P. Sheetz, "Membrane expansion increases endocytosis rate during mitosis," Journal of Cell Biology, vol. 144, no. 3, pp. 497-506, 1999. 


\section{Bibliography}

[10] D. Raucher and M. P. Sheetz, "Cell spreading and lamellipodial extension rate is regulated by membrane tension," Journal of Cell Biology, vol. 148, no. 1, pp. 127136, 2000.

[11] R. M. Hochmuth and W. D. Marcus, "Membrane tethers formed from blood cells with available area and determination of their adhesion energy," Biophysical Journal, vol. 82, no. 6, pp. 2964-2969, 2002.

[12] D. A. Fletcher and R. D. Mullins, "Cell mechanics and the cytoskeleton," Nature, vol. 463, pp. 485-492, 2010.

[13] K. C. Holmes, D. Popp, W. Gebhard, and W. Kabsch, "Atomic model of the actin filament," Nature, vol. 347, no. 6288, pp. 44-49, 1990.

[14] T. D. Pollard, "Rate constants for the reactions of ATP-and ADP-actin with the ends of actin filaments," The Journal of cell biology, vol. 103, no. 6, pp. 27472754, 1986.

[15] T. D. Pollard and J. A. Cooper, "Actin and actin-binding proteins. A critical evaluation of mechanisms and functions," Annual Review of Biochemistry, vol. 55, pp. 987-1035, 1986.

[16] J. Käs, H. Strey, J. X. Tang, D. Finger, R. Ezzell, E. Sackmann, and P. A. Janmey, "F-actin, a model polymer for semiflexible chains in dilute, semidilute, and liquid crystalline solutions," Biophysical Journal, vol. 70, no. 2, pp. 609-625, 1996.

[17] R. Sanghvi-Shah and G. F. Weber, "Intermediate filaments at the junction of mechanotransduction, migration and development," Frontiers in Cell and Developmental Biology, vol. 5, pp. 1-19, 2017.

[18] F. Gittes, B. Mickey, J. Nettleton, and J. Howard, "Flexural rigidity of microtubules and actin filaments measured from thermal fluctuations in shape," Journal of Cell Biology, vol. 120, no. 4, pp. 923-934, 1993.

[19] J. Howard, Mechanics of motor proteins and the cytoskeleton. Sinauer, 2001. 
[20] L. Blanchoin, R. Boujemaa-Paterski, C. Sykes, and J. Plastino, "Actin dynamics, architecture and mechanics in cell motility," Physiological Reviews, vol. 94, pp. 235-263, 2014.

[21] M. Algrain, O. Turunen, A. Vaheri, D. Louvard, and M. Arpin, "Ezrin contains cytoskeleton and membrane binding domains accounting for its proposed role as a membrane-cytoskeletal linker," The Journal of cell biology, vol. 120, no. 1, pp. 129-139, 1993.

[22] C. L. Leung, K. J. Green, and R. K. Liem, "Plakins: A family of versatile cytolinker proteins," Trends in Cell Biology, vol. 12, no. 1, pp. 37-45, 2002.

[23] N. Morone, T. Fujiwara, K. Murase, R. S. Kasai, H. Ike, S. Yuasa, J. Usukura, and A. Kusumi, "Three-dimensional reconstruction of the membrane skeleton at the plasma membrane interface by electron tomography," Journal of Cell Biology, vol. 174, no. 6, pp. 851-862, 2006.

[24] B. R. Brückner, A. Pietuch, S. Nehls, J. Rother, and A. Janshoff, "Ezrin is a major regulator of membrane tension in epithelial cells," Scientific Reports, vol. 5, p. $14700,2015$.

[25] B. M. Chung, J. D. Rotty, and P. A. Coulombe, "Networking galore: intermediate filaments and cell migration," Current Opinion in Cell Biology, vol. 25, no. 5, pp. 600-612, 2013.

[26] J. Block, V. Schroeder, P. Pawelzyk, N. Willenbacher, and S. Köster, "Physical properties of cytoplasmic intermediate filaments," Biochimica et Biophysica Acta - Molecular Cell Research, vol. 1853, no. 11, pp. 3053-3064, 2015.

[27] R. Windoffer, M. Beil, T. M. Magin, and R. E. Leube, "Cytoskeleton in motion: The dynamics of keratin intermediate filaments in epithelia," Journal of Cell Biology, vol. 194, no. 5, pp. 669-678, 2011.

[28] N. M. Kumar and N. B. Gilula, "The gap junction communication channel," Cell, vol. 84, no. 3, pp. 381-388, 1996. 


\section{Bibliography}

[29] H. Chiba, M. Osanai, M. Murata, T. Kojima, and N. Sawada, "Transmembrane proteins of tight junctions," Biochimica et Biophysica Acta Biomembranes, vol. 1778 , no. 3, pp. 588-600, 2008.

[30] L. L. Mitic and J. M. Anderson, "Molecular architecture of tight junctions," Annual Review of Physiology., vol. 60, pp. 1211-42, 1998.

[31] B. Gumbiner, "Structure, biochemistry, and assembly of epithelial tight junctions," The American Journal of Physiology, vol. 253, no. 6, pp. C749-C758, 1987.

[32] B. Gumbiner, "Generation and maintenance of epithelial cell polarity," Current Opinion in Cell Biology, vol. 2, pp. 881-887, 1990.

[33] J. L. Madara, "Intestinal absorptive cell tight junctions are linked to cytoskeleton," The American journal of physiology, vol. 253, no. 1 Pt 1, pp. C171-C175, 1987.

[34] O. J. Harrison, X. Jin, S. Hong, F. Bahna, G. Ahlsen, J. Brasch, Y. Wu, J. Vendome, K. Felsovalyi, C. M. Hampton, R. B. Troyanovsky, A. Ben-Shaul, J. Frank, S. M. Troyanovsky, L. Shapiro, and B. Honig, "The extracellular architecture of adherens junctions revealed by crystal structures of type I cadherins," Structure, vol. 19, no. 2, pp. 244-256, 2011.

[35] M. Takeichi, K. Hatta, A. Nose, and A. Nagafuchi, "Identification of a gene family of cadherin cell adhesion molecules," Cell Differentiation and Development, vol. 25, pp. 91-94, 1988.

[36] C. Bertocchi, Y. Wang, A. Ravasio, Y. Hara, Y. Wu, T. Sailov, M. A. Baird, M. W. Davidson, R. Zaidel-Bar, Y. Toyama, B. Ladoux, R. Mege, and P. Kanchanawong, "Nanoscale architecture of cadherin-based cell adhesions," Nature Cell Biology, vol. 19, no. 1, pp. 28-37, 2017.

[37] K. Maki, S. W. Han, Y. Hirano, S. Yonemura, T. Hakoshima, and T. Adachi, "Mechano-adaptive sensory mechanism of $\alpha$-catenin under tension," Scientific Reports, vol. 6, no. April, pp. 1-9, 2016.

[38] S. Yonemura, M. Itoh, A. Nagafuchi, and S. Tsukita, "Cell-to-cell adherens junction formation and actin filament organization: similarities and differences between 
non-polarized fibroblasts and polarized epithelial cells," Journal of Cell Science, vol. 108, pp. 127-142, 1995.

[39] S. Yonemura, Y. Wada, T. Watanabe, A. Nagafuchi, and M. Shibata, " $\alpha$-Catenin as a tension transducer that induces adherens junction development," Nature Cell Biology, vol. 12, no. 6, pp. 533-542, 2010.

[40] M. Takeichi, "Dynamic contacts: rearranging adherens junctions to drive epithelial remodelling," Nature reviews. Molecular cell biology, vol. 15, no. 6, pp. 397-410, 2014.

[41] B. Baum and M. Georgiou, "Dynamics of adherens junctions in epithelial establishment, maintenance, and remodeling," Journal of Cell Biology, vol. 192, no. 6, pp. 907-917, 2011.

[42] S. Pokutta and W. I. Weis, "Structure and mechanism of cadherins and catenins in cell-cell contacts," Annual Review of Cell and Developmental Biology, vol. 23, no. 1, pp. 237-261, 2007.

[43] M. D. Kottke, "The desmosome: cell science lessons from human diseases," Journal of Cell Science, vol. 119, no. 5, pp. 797-806, 2006.

[44] L. Borradori and A. Sonnenberg, "Structure and function of hemidesmosomes: More than simple adhesion complexes," Journal of Investigative Dermatology, vol. 112, no. 4, pp. 411-418, 1999.

[45] S. Wallis, S. Lloyd, I. Wise, G. Ireland, T. P. Fleming, and D. Garrod, "The $\alpha$ isoform of protein kinase $C$ is involved in signaling the response of desmosomes to wounding in cultured epithelial cells," Molecular Biology of the Cell, vol. 11, no. 3, pp. 1077-1092, 2000.

[46] D. R. Garrod, M. Y. Berika, W. F. Bardsley, D. Holmes, and L. Tabernero, "Hyperadhesion in desmosomes: its regulation in wound healing and possible relationship to cadherin crystal structure," Journal of Cell Science, vol. 118, no. 24, pp. 57435754, 2005.

[47] E. Zamir and B. Geiger, "Molecular complexity and dynamics of cell-matrix adhesions," Journal of Cell Science, vol. 114, no. 20, pp. 3583-90, 2001. 


\section{Bibliography}

[48] P. Kanchanawong, G. Shtengel, A. M. Pasapera, E. B. Ramko, M. W. Davidson, H. F. Hess, and C. M. Waterman, "Nanoscale architecture of integrin-based cell adhesions," Nature, vol. 468, no. 7323, pp. 580-584, 2010.

[49] A. D. Bershadsky, N. Q. Balaban, and B. Geiger, "Adhesion-dependent cell mechanosensitivity," Annual review of cell and developmental biology, vol. 19, pp. 677-95, 2003.

[50] A. J. Ridley, M. A. Schwartz, K. Burridge, R. A. Firtel, M. H. Ginsberg, G. Borisy, J. T. Parsons, and A. R. Horwitz, "Cell migration: integrating signals from front to back," Science, vol. 302, pp. 1704-1710, 2003.

[51] C. S. Chen, "Mechanotransduction - a field pulling together?," Journal of Cell Science, vol. 121, pp. 3285-3292, 2008.

[52] A. R. Harris, A. Daeden, and G. T. Charras, "Formation of adherens junctions leads to the emergence of a tissue-level tension in epithelial monolayers," Journal of Cell Science, pp. 2507-2517, 2014.

[53] P. O. Strale, L. Duchesne, G. Peyret, L. Montel, T. Nguyen, E. Png, R. Tampé, S. Troyanovsky, S. Hénon, B. Ladoux, and R. M. Mège, "The formation of ordered nanoclusters controls cadherin anchoring to actin and cell-cell contact fluidity," Journal of Cell Biology, vol. 210, no. 2, pp. 333-346, 2015.

[54] Y. Wu, P. Kanchanawong, and R. Zaidel-bar, "Actin-delimited adhesionindependent clustering of E-cadherin forms the nanoscale building blocks of adherens junctions," Developmental Cell, vol. 32, no. 2, pp. 139-154, 2015.

[55] J. A. Tunggal, I. Helfrich, A. Schmitz, H. Schwarz, D. Günzel, M. Fromm, R. Kemler, T. Krieg, and C. M. Niessen, "E-cadherin is essential for in vivo epidermal barrier function by regulating tight junctions," EMBO Journal, vol. 24, no. 6, pp. 1146-1156, 2005.

[56] B. Gumbiner, B. Stevenson, and A. Grimaldi, "The role of the cell adhesion molecular uvomorulin in the formation and maintenance of the epithelial junctional complex," Journal of Cell Biology, vol. 107, no. October, pp. 1575-1587, 1988. 
[57] A. Rajasekaran, M. Hojo, T. Huima, and E. Rodriguez-Boulan, "Catenins and Zonula Occludins-1 form a complex during early stages in the assembly of tight junctions," Journal of Cell Biology, vol. 132, no. 3, pp. 451-463, 1996.

[58] C. T. Capaldo and I. G. Macara, "Depletion of E-cadherin disrupts establishment but not maintenance of cell junctions in Madin-Darby canine kidney epithelial cells," Molecular biology of the cell, vol. 18, no. 1, pp. 189-200, 2007.

[59] M. Rübsam, A. F. Mertz, A. Kubo, S. Marg, C. Jüngst, G. Goranci-Buzhala, A. C. Schauss, V. Horsley, E. R. Dufresne, M. Moser, W. Ziegler, M. Amagai, S. A. Wickström, and C. M. Niessen, "E-cadherin integrates mechanotransduction and EGFR signaling to control junctional tissue polarization and tight junction positioning," Nature Communications, vol. 8, no. 1, pp. 1-15, 2017.

[60] O. Nekrasova and K. J. Green, "Desmosome assembly and dynamics," Trends in Cell Biology, vol. 23, no. 11, pp. 537-546, 2013.

[61] O. Shafraz, M. Rübsam, S. N. Stahley, A. Caldara, A. P. Kowalczyk, C. M. Niessen, and S. Sivasankar, "E-cadherin binds to desmoglein to facilitate desmosome assembly," eLife, vol. 7, p. e37629, 2018.

[62] S. Kaliman, C. Jayachandran, F. Rehfeldt, and A. S. Smith, "Novel growth regime of MDCK II model tissues on soft substrates," Biophysical Journal, vol. 106, no. 7 , pp. 25-28, 2014.

[63] C. L. Adams, Y.-T. Chen, S. J. Smith, and W. James Nelson, "Mechanisms of epithelial cell-cell adhesion and cell compaction revealed by high-resolution tracking of E-cadherin- green fluorescent protein," The Journal of Cell Biology, vol. 142, no. 4, pp. 1105-1119, 1998.

[64] C. R. Gaush, W. L. Hard, and T. F. Smith, "Characterization of an established line of canine kidney cells (mdck)," Proceedings of the Society for Experimental Biology and Medicine, vol. 122, no. 3, pp. 931-935, 1966.

[65] J. D. Valentich, "Morphological similarities between the dog kidney cell line MDCK and the mammalian cortical collecting tubule," Annals of the New York Academy of Sciences, vol. 372, no. 1, pp. 384-405, 1981. 


\section{Bibliography}

[66] J. D. Dukes, P. Whitley, and A. D. Chalmers, "The MDCK variety pack: Choosing the right strain," BMC Cell Biology, vol. 12, no. 1, p. 43, 2011.

[67] B. Rothen-Rutishauser, S. D. Krämer, A. Braun, M. Günthert, and H. WunderliAllenspach, "MDCK cell cultures as an epithelial in vitro model: cytoskeleton and tight junctions as indicators for the definition of age-related stages by confocal microscopy," Pharmaceutical Research, vol. 15, no. 7, pp. 964-971, 1998.

[68] J. Balcarova-Ständer, S. E. Pfeiffer, S. D. Fuller, and K. Simons, "Development of cell surface polarity in the epithelial Madin-Darby canine kidney (MDCK) cell line," The EMBO journal, vol. 3, no. 11, pp. 2687-94, 1984.

[69] L. Petitjean, M. Reffay, E. Grasland-Mongrain, M. Poujade, B. Ladoux, A. Buguin, and P. Silberzan, "Velocity fields in a collectively migrating epithelium," Biophysical Journal, vol. 98, no. 9, pp. 1790-1800, 2010.

[70] D. T. Tambe, C. C. Hardin, T. E. Angelini, K. Rajendran, C. Y. Park, X. SerraPicamal, E. H. Zhou, M. H. Zaman, J. P. Butler, D. A. Weitz, J. J. Fredberg, and $X$. Trepat, "Collective cell guidance by cooperative intercellular forces," Nature Materials, vol. 10, no. 6, pp. 469-475, 2011.

[71] P. Vijayaraj, C. Kröger, U. Reuter, R. Windoffer, R. E. Leube, and T. M. Magin, "Keratins regulate protein biosynthesis through localization of GLUT1 and -3 upstream of AMP kinase and Raptor," Journal of Cell Biology, vol. 187, no. 2, pp. 175-184, 2009.

[72] L. Ramms, G. Fabris, R. Windoffer, N. Schwarz, R. Springer, C. Zhou, J. Lazar, S. Stiefel, N. Hersch, U. Schnakenberg, T. M. Magin, R. E. Leube, R. Merkel, and B. Hoffmann, "Keratins as the main component for the mechanical integrity of keratinocytes," Proceedings of the National Academy of Sciences of the United States of America, vol. 110, no. 46, pp. 18513-8, 2013.

[73] F. Loschke, K. Seltmann, J. E. Bouameur, and T. M. Magin, "Regulation of keratin network organization," Current Opinion in Cell Biology, vol. 32, pp. 56-64, 2015. 
[74] S. Mazzalupo, P. Wong, P. Martin, and P. Coulombe, "Role for keratins 6 and 17 during wound closure in embryonic mouse skin," Developmental Dynamics, vol. 226, no. 2, pp. 356-365, 2003.

[75] A. J. Engler, S. Sen, H. L. Sweeney, and D. E. Discher, "Matrix elasticity directs stem cell lineage specification," Cell, vol. 126, no. 4, pp. 677-689, 2006.

[76] C. M. Lo, H. B. Wang, M. Dembo, and Y. L. Wang, "Cell movement is guided by the rigidity of the substrate," Biophysical Journal, vol. 79, no. 1, pp. 144-152, 2000.

[77] T. Iskratsch, H. Wolfenson, and M. P. Sheetz, "Appreciating force and shape the rise of mechanotransduction in cell biology," Nature Reviews Molecular Cell Biology, vol. 15, no. 12, pp. 825-833, 2014.

[78] D. E. Leckband, Q. le Duc, N. Wang, and J. de Rooij, "Mechanotransduction at cadherin-mediated adhesions," Current Opinion in Cell Biology, vol. 23, pp. 523530, 2011.

[79] K. A. Beningo, M. Dembo, I. Kaverina, J. V. Small, and Y. L. Wang, "Nascent focal adhesions are responsible for the generation of strong propulsive forces in migrating fibroblasts," Journal of Cell Biology, vol. 153, no. 4, pp. 881-887, 2001.

[80] Z. Liu, J. L. Tan, D. M. Cohen, M. T. Yang, N. J. Sniadecki, S. A. Ruiz, C. M. Nelson, and C. S. Chen, "Mechanical tugging force regulates the size of cell-cell junctions," Proceedings of the National Academy of Sciences of the United States of America, vol. 107, pp. 9944-9949, 2010.

[81] C. Grashoff, B. D. Hoffman, M. D. Brenner, R. Zhou, M. Parsons, M. T. Yang, M. A. McLean, S. G. Sligar, C. S. Chen, T. Ha, and M. A. Schwartz, "Measuring mechanical tension across vinculin reveals regulation of focal adhesion dynamics," Nature, vol. 466, no. 7303, pp. 263-6, 2010.

[82] N. Borghi, M. Sorokina, O. G. Shcherbakova, W. I. Weis, B. L. Pruitt, W. J. Nelson, and a. R. Dunn, "E-cadherin is under constitutive actomyosin-generated tension that is increased at cell-cell contacts upon externally applied stretch," 
Bibliography

Proceedings of the National Academy of Sciences, vol. 109, no. 31, pp. 1256812573, 2012.

[83] A. K. Barry, H. Tabdili, I. Muhamed, J. Wu, N. Shashikanth, G. Gomez, A. Yap, C. J. Gottardi, J. de Rooij, N. Wang, and D. E. Leckband, " $\alpha$-catenin cytomechanics-role in cadherin-dependent adhesion and mechanotransduction," Journal of Cell Science, vol. 127, pp. 1779-91, 2014.

[84] W. H. Ziegler, R. C. Liddington, and D. R. Critchley, "The structure and regulation of vinculin," Trends in Cell Biology, vol. 16, no. 9, pp. 453-460, 2006.

[85] D. Choquet, D. P. Felsenfeld, and M. P. Sheetz, "Extracellular matrix rigidity causes strengthening of integrin- cytoskeleton linkages," Cell, vol. 88, no. 1, pp. 39-48, 1997.

[86] Q. Le Duc, Q. Shi, I. Blonk, A. Sonnenberg, N. Wang, D. E. Leckband, and J. De Rooij, "Vinculin potentiates E-cadherin mechanosensing and is recruited to actin-anchored sites within adherens junctions in a myosin II-dependent manner," Journal of Cell Biology, vol. 189, no. 7, pp. 1107-1115, 2010.

[87] C. D. Buckley, J. Tan, K. L. Anderson, D. Hanein, N. Volkmann, W. I. Weis, W. J. Nelson, and A. R. Dunn, "The minimal cadherin-catenin complex binds to actin filaments under force," Science, vol. 346, no. 6209, 2014.

[88] I. Muhamed, J. Wu, P. Sehgal, X. Kong, A. Tajik, N. Wang, and D. E. Leckband, "E-Cadherin-mediated force transduction signals regulate global cell mechanics," Journal of Cell Science, vol. 129, pp. 1843-1854, 2016.

[89] V. Maruthamuthu, B. Sabass, U. S. Schwarz, and M. L. Gardel, "Cell-ECM traction force modulates endogenous tension at cell-cell contacts," Proceedings of the National Academy of Sciences of the United States of America, vol. 108, pp. 4708-4713, 2011.

[90] B. Noethel, L. Ramms, G. Dreissen, M. Hoffmann, R. Springer, M. Rübsam, W. H. Ziegler, C. M. Niessen, R. Merkel, and B. Hoffmann, "Transition of responsive mechanosensitive elements from focal adhesions to adherens junctions 
upon epithelial differentiation," Molecular Biology of the Cell, vol. 49, no. 0 , pp. mbc.E17-06-0387, 2018.

[91] C. Collins and W. J. Nelson, "Running with neighbors: coordinating cell migration and cell-cell adhesion," Current Opinion in Cell Biology, vol. 36, pp. 62-70, 2015.

[92] D. Garrod and M. Chidgey, "Desmosome structure, composition and function," Biochimica et Biophysica Acta - Biomembranes, vol. 1778, no. 3, pp. 572-587, 2008.

[93] S. Getsios, A. C. Huen, and K. J. Green, "Working out the strength and flexibility of desmosomes," Nature Reviews Molecular Cell Biology, vol. 5, no. 4, pp. 271281, 2004.

[94] N. Osmani and M. Labouesse, "Remodeling of keratin-coupled cell adhesion complexes," Current Opinion in Cell Biology, vol. 32, pp. 30-38, 2015.

[95] M. Hatzfeld, R. Keil, and T. M. Magin, "Desmosomes and intermediate filaments: Their consequences for tissue mechanics," Cold Spring Harbor Perspectives in Biology, vol. 9, no. 6, 2017.

[96] J. A. Broussard, R. Yang, C. Huang, S. S. P. Nathamgari, A. M. Beese, L. M. Godsel, M. H. Hegazy, S. Lee, F. Zhou, N. J. Sniadecki, K. J. Green, and H. D. Espinosa, "The desmoplakin-intermediate filament linkage regulates cell mechanics," Molecular Biology of the Cell, vol. 28, no. 23, pp. 3156-3164, 2017.

[97] K. J. Cavanaugh, J. Oswari, and S. S. Margulies, "Role of stretch on tight junction structure in alveolar epithelial cells," American Journal of Respiratory Cell and Molecular Biology, vol. 25, pp. 584-591, 2001.

[98] J. L. Madara, R. Moore, and S. Carlson, "Alteration of intestinal tight junction structure and permeability by cytoskeletal contraction," The American journal of physiology, vol. 253, no. 6 Pt 1, pp. C854-61, 1987.

[99] B. R. Brückner and A. Janshoff, "Importance of integrity of cell-cell junctions for the mechanics of confluent mdck ii cells," Scientific Reports, vol. 8, no. 1, p. 14117, 2018. 
Bibliography

[100] A. X. Cartagena-Rivera, C. M. Van Itallie, J. M. Anderson, and R. S. Chadwick, "Apical surface supracellular mechanical properties in polarized epithelium using noninvasive acoustic force spectroscopy," Nature Communications, vol. 8, no. 1 , p. 1030, 2017.

[101] L. Kreplak, H. Bär, J. F. Leterrier, H. Herrmann, and U. Aebi, "Exploring the mechanical behavior of single intermediate filaments," Journal of Molecular Biology, vol. 354, no. 3, pp. 569-577, 2005.

[102] C. P. Brangwynne, F. C. MacKintosh, N. A. Kumar, S.and Geisse, J. Talbot, L. Mahadevan, K. K. Parker, D. E. Ingber, and D. A. Weitz, "Microtubules can bear enhanced compressive loads in living cells because of lateral reinforcement," Journal of Cell Biology, vol. 173, no. 5, pp. 733-741, 2006.

[103] D. E. Ingber, "Cellular tensegrity: defining new rules of biological design that govern the cytoskeleton," Journal of Cell Science, vol. 104, pp. 613-627, 1993.

[104] L. Deng, X. Trepat, J. P. Butler, E. Millet, K. G. Morgan, D. A. Weitz, and J. J. Fredberg, "Fast and slow dynamics of the cytoskeleton," Nature Materials, vol. 5, no. 8, pp. 636-640, 2006.

[105] J. H.-C. Wang, P. Goldschmidt-Clermont, J. Wille, and F. C.-P. Yin, "Specificity of endothelial cell reorientation in response to cyclic mechanical stretching," Journal of Biomechanics, vol. 34, no. 12, pp. 1563-1572, 2001.

[106] U. Faust, N. Hampe, W. Rubner, N. Kirchgessner, S. Safran, B. Hoffmann, and R. Merkel, "Cyclic stress at mhz frequencies aligns fibroblasts in direction of zero strain," PLOS ONE, vol. 6, no. 12, pp. 1-16, 2011.

[107] A. Zemel, F. Rehfeldt, A. E. X. Brown, D. E. Discher, and S. A. Safran, "Optimal matrix rigidity for stress fiber polarization in stem cells," Nature Physics, vol. 6, no. 6 , pp. 468-473, 2010.

[108] K. Ohashi, S. Fujiwara, and K. Mizuno, "Roles of the cytoskeleton, cell adhesion and rho signalling in mechanosensing and mechanotransduction," Journal of Biochemistry, vol. 161, no. 3, pp. 245-254, 2017. 
[109] I. Kaverina, O. Krylyshkina, K. Beningo, K. Anderson, Y.-L. Wang, and J. V. Small, "Tensile stress stimulates microtubule outgrowth in living cells," Journal of Cell Science, vol. 115, no. Pt 11, pp. 2283-2291, 2002.

[110] S. Etienne-Manneville, "Microtubules in cell migration," Annual Review of Cell and Developmental Biology, vol. 29, no. 1, pp. 471-499, 2013.

[111] P. A. Janmey, U. Euteneuer, P. Traub, and M. Schliwa, "Viscoelastic properties of vimentin compared with other filamentous biopolymer networks," Journal of Cell Biology, vol. 113, no. 1, pp. 155-160, 1991.

[112] J. Block, H. Witt, A. Candelli, E. J. Peterman, G. J. Wuite, A. Janshoff, and S. Köster, "Nonlinear loading-rate-dependent force response of individual vimentin intermediate filaments to applied strain," Physical Review Letters, vol. 118, no. 4, pp. 1-5, 2017.

[113] K. Seltmann, A. W. Fritsch, J. Käs, and T. M. Magin, "Keratins significantly contribute to cell stiffness and impact invasive behavior," Proceedings of the $\mathrm{Na}$ tional Academy of Sciences of the United States of America, vol. 110, no. 46, pp. 18507-12, 2013.

[114] K. Seltmann, W. Roth, C. Kröger, F. Loschke, M. Lederer, S. Hüttelmaier, and T. M. Magin, "Keratins mediate localization of hemidesmosomes and repress cell motility," Journal of Investigative Dermatology, vol. 133, no. 1, pp. 181-190, 2012.

[115] G. F. Weber, M. A. Bjerke, and D. W. DeSimone, "A Mechanoresponsive Cadherin-Keratin Complex Directs Polarized Protrusive Behavior and Collective Cell Migration," Developmental Cell, vol. 22, no. 1, pp. 104-115, 2012.

[116] P. Wong and P. A. Coulombe, "Loss of keratin 6 (K6) proteins reveals a function for intermediate filaments during wound repair," Journal of Cell Biology, vol. 163, no. 2, pp. 327-337, 2003.

[117] R. Sunyer, V. Conte, J. Escribano, A. Elosegui-Artola, A. Labernadie, L. Valon, D. Navajas, J. M. García-Aznar, J. J. Muñoz, P. Roca-Cusachs, and X. Trepat, 
Bibliography

"Collective cell durotaxis emerges from long-range intercellular force transmission," Science, vol. 353, no. 6304, pp. 1157-1161, 2016.

[118] M. Reffay, M. C. Parrini, O. Cochet-Escartin, B. Ladoux, A. Buguin, S. Coscoy, F. Amblard, J. Camonis, and P. Silberzan, "Interplay of RhoA and mechanical forces in collective cell migration driven by leader cells," Nature Cell Biology, vol. 16, no. 3, pp. 217-223, 2014.

[119] T. Das, K. Safferling, S. Rausch, N. Grabe, H. Boehm, and J. P. Spatz, "A molecular mechanotransduction pathway regulates collective migration of epithelial cells," Nature Cell Biology, vol. 17, no. 3, pp. 276-287, 2015.

[120] X. Serra-Picamal, V. Conte, R. Vincent, E. Anon, D. T. Tambe, E. Bazellieres, J. P. Butler, J. J. Fredberg, and X. Trepat, "Mechanical waves during tissue expansion," Nature Physics, vol. 8, no. 8, pp. 628-634, 2012.

[121] S. R. K. Vedula, M. C. Leong, T. L. Lai, P. Hersen, A. J. Kabla, C. T. Lim, and B. Ladoux, "Emerging modes of collective cell migration induced by geometrical constraints," Proceedings of the National Academy of Sciences of the United States of America, vol. 109, no. 32, pp. 12974-9, 2012.

[122] E. Bazellières, V. Conte, A. Elosgui-Artola, X. Serra-Picamal, M. BintanelMorcillo, P. Roca-Cusachs, J. J. Muñoz, M. Sales-Pardo, R. Guimerà, and $X$. Trepat, "Control of cell-cell forces and collective cell dynamics by the intercellular adhesome," Nature Cell Biology, vol. 17, no. 4, 2015.

[123] B. Ladoux and R. M. Mège, "Mechanobiology of collective cell behaviours," Nature Reviews Molecular Cell Biology, vol. 18, no. 12, pp. 743-757, 2017.

[124] S. R. K. Vedula, H. Hirata, M. H. Nai, A. Brugués, Y. Toyama, X. Trepat, C. T. Lim, and B. Ladoux, "Epithelial bridges maintain tissue integrity during collective cell migration," Nature Materials, vol. 13, no. 1, pp. 87-96, 2014.

[125] L. Coburn, I.-M. Schouwenaar, H. Lopez, A. S. Yap, V. Lobaskin, and G. A. Gomez, "Contact inhibition of locomotion and junctional mechanics guide collective cell behavior in epithelial wound repair," ArXiv e-print 1702.01578, 2017. 
[126] A. R. Harris, L. Peter, J. Bellis, B. Baum, A. J. Kabla, and G. T. Charras, "Characterizing the mechanics of cultured cell monolayers," Proceedings of the National Academy of Sciences, vol. 109, no. 41, pp. 16449-16454, 2012.

[127] G. T. Eisenhoffer, P. D. Loftus, M. Yoshigi, H. Otsuna, C.-B. Chien, P. A. Morcos, and J. Rosenblatt, "Crowding induces live cell extrusion to maintain homeostatic cell numbers in epithelia," Nature, vol. 484, no. 7395, pp. 546-549, 2013.

[128] C. Guillot and T. Lecuit, "Mechanics of epithelial tissue homeostasis and morphogenesis," Science, vol. 340, no. June, pp. 1185-1189, 2013.

[129] R. Farhadifar, J. C. Röper, B. Aigouy, S. Eaton, and F. Jülicher, "The influence of cell mechanics, cell-cell interactions, and proliferation on epithelial packing," Current Biology, vol. 17, no. 24, pp. 2095-2104, 2007.

[130] A. Noppe, A. P. Roberts, A. S. Yap, G. A. Gomez, and Z. Neufeld, "Modelling wound closure in an epithelial cell sheet using the Cellular Potts Model," Integrative Biology, vol. 7, pp. 1253-1264, 2015.

[131] G.-K. Xu, Y. Liu, and B. Li, "How do changes at the cell level affect the mechanical properties of epithelial monolayers?," Soft Matter, vol. 11, no. 45, pp. 8782-8788, 2015.

[132] Y. A. Miroshnikova, H. Q. Le, D. Schneider, T. Thalheim, M. Rübsam, N. Bremicker, J. Polleux, N. Kamprad, M. Tarantola, I. Wang, M. Balland, C. M. Niessen, J. Galle, and S. A. Wickström, "Adhesion forces and cortical tension couple cell proliferation and differentiation to drive epidermal stratification," Nature Cell Biology, vol. 20, no. 1, p. 69, 2018.

[133] C. De Pascalis, C. Pérez-González, S. Seetharaman, B. Boëda, B. Vianay, M. Burute, C. Leduc, N. Borghi, X. Trepat, and S. Etienne-Manneville, "Intermediate filaments control collective migration by restricting traction forces and sustaining cell?cell contacts," The Journal of Cell Biology, p. jcb.201801162, 2018.

[134] P. R. Sonavane, C. Wang, B. Dzamba, G. F. Weber, A. Periasamy, and D. W. DeSimone, "Mechanical and signaling roles for keratin intermediate filaments in 


\section{Bibliography}

the assembly and morphogenesis of mesendoderm tissue at gastrulation," Development, no. October, p. dev.155200, 2017.

[135] S. Köster, D. A. Weitz, R. D. Goldman, U. Aebi, and H. Herrmann, "Intermediate filament mechanics in vitro and in the cell: From coiled coils to filaments, fibers and networks," Current Opinion in Cell Biology, vol. 32, pp. 82-91, 2015.

[136] S. Karsch, D. Kong, J. Großhans, and A. Janshoff, "Single-cell defects cause a long-range mechanical response in a confluent epithelial cell layer," Biophysical Journal, vol. 113, no. 12, pp. 2601-2608, 2017.

[137] T. J. Shaw and P. Martin, "Wound repair at a glance," Journal of Cell Science, vol. 122, no. 18, pp. 3209-3213, 2009.

[138] A. M. Marchiando, W. V. Graham, and J. R. Turner, "Epithelial barriers in homeostasis and disease," Annual review of pathology, vol. 5, pp. 119-144, 2010.

[139] S. R. K. Vedula, G. Peyret, I. Cheddadi, T. Chen, A. Brugues, H. Hirata, H. LopezMenendez, Y. Toyama, L. Neves de Almeida, X. Trepat, C. T. Lim, and B. Ladoux, "Mechanics of epithelial closure over non-adherent environments," Nature communications, vol. 6, p. 6111, 2015.

[140] E. Anon, X. Serra-Picamal, P. Hersen, N. C. Gauthier, M. P. Sheetz, X. Trepat, and B. Ladoux, "Cell crawling mediates collective cell migration to close undamaged epithelial gaps," Proceedings of the National Academy of Sciences of the United States of America, vol. 109, no. 27, pp. 10891-6, 2012.

[141] A. Ravasio, I. Cheddadi, T. Chen, T. Pereira, H. T. Ong, C. Bertocchi, A. Brugues, A. Jacinto, A. J. Kabla, Y. Toyama, X. Trepat, N. Gov, L. Neves de Almeida, and B. Ladoux, "Gap geometry dictates epithelial closure efficiency," Nature Communications, vol. 6, p. 7683, 2015.

[142] T. Omelchenko, J. M. Vasiliev, I. M. Gelfand, H. H. Feder, and E. M. Bonder, "Rho-dependent formation of epithelial leader cells during wound healing," Proceedings of the National Academy of Sciences of the United States of America, vol. 100, no. 19, pp. 10788-93, 2003. 
[143] M. Poujade, E. Grasland-Mongrain, A. Hertzog, J. Jouanneau, P. Chavrier, B. Ladoux, A. Buguin, and P. Silberzan, "Collective migration of an epithelial monolayer in response to a model wound," Proceedings of the National Academy of Sciences, vol. 104, no. 41, pp. 15988-15993, 2007.

[144] G. Fenteany, P. A. Janmey, and T. P. Stossel, "Signaling pathways and cell mechanics involved in wound closure by epithelial cell sheets," Current Biology, vol. 10, no. 14, pp. 831-838, 2000.

[145] P. Martin and J. Lewis, "Actin cables and epidermal movement in embryonic wound healing," Nature, vol. 360, no. 6400, pp. 179-183, 1992.

[146] W. M. Bement, P. Forscher, and M. S. Mooseker, "A novel cytoskeletal structure involved in purse string wound closure and cell polarity maintenance," Journal of Cell Biology, vol. 121, no. 3, pp. 565-578, 1993.

[147] Y. Danjo and I. K. Gipson, "Actin 'purse string' filaments are anchored by Ecadherin-mediated adherens junctions at the leading edge of the epithelial wound, providing coordinated cell movement," Journal of Cell Science, vol. 111, pp. 332332, 1998.

[148] P. Florian, T. Schöneberg, J. D. Schulzke, M. Fromm, and A. H. Gitter, "Singlecell epithelial defects close rapidly by an actinomyosin purse string mechanism with functional tight junctions," The Journal of physiology, vol. 545, pp. 485-99, 2002.

[149] D. Günzel, P. Florian, J. F. Richter, H. Troeger, J. D. Schulzke, M. Fromm, and A. H. Gitter, "Restitution of single-cell defects in the mouse colon epithelium differs from that of cultured cells," American journal of physiology. Regulatory, integrative and comparative physiology, vol. 290, no. 6, pp. R1496-507, 2006.

[150] S. Begnaud, T. Chen, D. Delacour, R. M. Mège, and B. Ladoux, "Mechanics of epithelial tissues during gap closure," Current Opinion in Cell Biology, vol. 42, pp. 52-62, 2016. 


\section{Bibliography}

[151] A. Brugues, E. Anon, V. Conte, J. H. Veldhuis, M. Gupta, J. Colombelli, J. Muñoz, G. W. Brodland, B. Ladoux, and X. Trepat, "Forces driving epithelial wound healing," Nature Physics, vol. 10, no. 1, pp. 683-690, 2014.

[152] J. Rosenblatt, M. C. Raff, and L. P. Cramer, "An epithelial cell destined for apoptosis signals its neighbors to extrude it by an actin- and myosin-dependent mechanism," Current Biology, vol. 11, no. 23, pp. 1847-1857, 2001.

[153] D. Kuipers, A. Mehonic, M. Kajita, L. Peter, Y. Fujita, T. Duke, G. T. Charras, and J. E. Gale, "Epithelial repair is a two-stage process driven first by dying cells and then by their neighbours," Journal of Cell Science, vol. 127, no. Pt 6, pp. 1229-41, 2014.

[154] J. M. Russo, "Distinct temporal-spatial roles for rho kinase and myosin light chain kinase in epithelial purse-string wound closure," Gastroenterology, vol. 14, no. 2, pp. 1-21, 2005.

[155] M. Tamada, T. D. Perez, W. J. Nelson, and M. P. Sheetz, "Two distinct modes of myosin assembly and dynamics during epithelial wound closure," Journal of Cell Biology, vol. 176, no. 1, pp. 27-33, 2007.

[156] D. Andrade and J. Rosenblatt, "Apoptotic regulation of epithelial cellular extrusion," Apoptosis, vol. 16, no. 5, pp. 491-501, 2011.

[157] Y. Gu and J. Rosenblatt, "New emerging roles for epithelial cell extrusion," Current Opinion in Cell Biology, vol. 24, no. 6, pp. 865-870, 2012.

[158] I. Pastar, O. Stojadinovic, N. C. Yin, H. Ramirez, A. G. Nusbaum, A. Sawaya, S. B. Patel, L. Khalid, R. R. Isseroff, and M. Tomic-Canic, "Epithelialization in Wound Healing: A Comprehensive Review," Advances in Wound Care, vol. 3, no. 7, pp. 445-464, 2014.

[159] C.-C. Liang, A. Y. Park, and J.-L. Guan, "In vitro scratch assay: a convenient and inexpensive method for analysis of cell migration in vitro," Nature protocols, vol. 2, no. 2, p. 329, 2007. 
[160] J. Colombelli, E. G. Reynaud, and E. H. K. Stelzer, "Investigating relaxation processes in cells and developing organisms: from cell ablation to cytoskeleton nanosurgery," Methods in Cell Biology, vol. 82, no. 06, pp. 267-291, 2007.

[161] D. B. Murphy, Fundamentals of light microscopy and electronic imaging. John Wiley \& Sons, 2002.

[162] M. D. Abràmoff, P. J. Magalhães, and S. J. Ram, "Image processing with imagej," Biophotonics international, vol. 11, no. 7, pp. 36-42, 2004.

[163] F. J. Segerer, P. J. F. Röttgermann, S. Schuster, A. Piera Alberola, S. Zahler, and J. O. Rädler, "Versatile method to generate multiple types of micropatterns," Biointerphases, vol. 11, no. 1, p. 011005, 2016.

[164] S. Nehls, "Cortical tension of cells: from apical membrane patches to patterned cells," Dissertation, University of Göttingen, 2018.

[165] G. Binnig, C. F. Quate, and C. Gerber, "Atomic force microscope," Physical review letters, vol. 56, no. 9, p. 930, 1986.

[166] J. L. Hutter and J. Bechhoefer, "Calibration of atomic-force microscope tips," Review of Scientific Instruments, vol. 64, no. 7, pp. 1868-1873, 1993.

[167] H. Hertz, "Über die Berührung fester elastischer Körper," Journal für die reine und angewandte Mathematik, vol. 92, pp. 156-171, 1881.

[168] I. N. Sneddon, "The relation between load and penetration in the axisymmetric boussinesq problem for a punch of arbitrary profile," International Journal of Engineering Science, vol. 3, no. 1, pp. 47-57, 1965.

[169] A. Hategan, R. Law, S. Kahn, and D. E. Discher, "Adhesively-tensed cell membranes: lysis kinetics and atomic force microscopy probing," Biophysical Journal, vol. 85, no. 4, pp. 2746-2759, 2003.

[170] S. Sen, S. Subramanian, and D. E. Discher, "Indentation and adhesive probing of a cell membrane with AFM: theoretical model and experiments," Biophysical Journal, vol. 89, no. 5, pp. 3203-3213, 2005. 
Bibliography

[171] C. E. Morris and U. Homann, "Cell surface area regulation and membrane tension," The Journal of Membrane Biology, vol. 179, no. 2, pp. 79-102, 2001.

[172] P. Sens and J. Plastino, "Membrane tension and cytoskeleton organization in cell motility," Journal of Physics: Condensed Matter, vol. 27, no. 27, p. 273103, 2015.

[173] I. Giaever and C. R. Keese, "Micromotion of mammalian cells measured electrically," Proceedings of the National Academy of Sciences of the United States of America, vol. 88, no. 17, pp. 7896-7900, 1991.

[174] C. M. Lo, C. R. Keese, and I. Giaever, "Monitoring motion of confluent cells in tissue culture," Experimental Cell Research, vol. 204, no. 1, pp. 102-109, 1993.

[175] C. M. Lo, C. R. Keese, and I. Giaever, "Impedance analysis of MDCK cells measured by electric cell-substrate impedance sensing," Biophysical Journal, vol. 69, no. 6, pp. 2800-2807, 1995.

[176] R. Zeitler and P. Fromherz, "The thermal voltage fluctuations in the planar corecoat conductor of a neuron-semiconductor interface," Langmuir, vol. 29, no. 20, pp. 6084-6090, 2013.

[177] N. A. Abaza, J. Leighton, and S. G. Schultz, "Effects of ouabain on the function and structure of a cell line (MDCK) derived from canine kidney: I. Light microscopic observations of monolayer growth," In Vitro: Journal of the Tissue Culture Association, vol. 10, no. 3, pp. 172-183, 1974.

[178] A. Ettinger and T. Wittmann, "Flourescence live cell imaging," Methods in Cell Biology, vol. 123, pp. 77-94, 2015.

[179] J. D. Stoien and R. J. Wang, "Effect of near-ultraviolet and visible light on mammalian cells in culture II. Formation of toxic photoproducts in tissue culture medium by blacklight," Proceedings of the National Academy of Sciences of the United States of America, vol. 71, no. 10, pp. 3961-5, 1974.

[180] J. S. Zigler Jr., J. L. Lepe-Zuniga, B. Vistica, and I. Gery, "Analysis of the cytotoxic effects of light-exposed hepes-containing culture medium," In Vitro Cellular \& Developmental Biology, vol. 21, no. 5, pp. 282-287, 1985. 
[181] L. Hoheisel, "Temperature dependency of the mechanical properties of MDCK II cells," Bachelor Thesis, University of Göttingen, 2018.

[182] P. Schröder, "Formation of cell polarity in epithelial cells: a confocal microscopy study," Report, University of Göttingen, 2016.

[183] K. Simons and S. D. Fuller, "Cell surface polarity in epithelia," Annual Review of Cell Biology, vol. 1, pp. 243-288, 1985.

[184] D. Meder, A. Shevchenko, K. Simons, and J. Füllekrug, "Gp135/podocalyxin and nherf-2 participate in the formation of a preapical domain during polarization of mdck cells," The Journal of cell biology, vol. 168, no. 2, pp. 303-313, 2005.

[185] G. K. Ojakian and R. Schwimmer, "The polarized distribution of an apical cell surface glycoprotein is maintained by interactions with the cytoskeleton of madindarby canine kidney cells," The Journal of cell biology, vol. 107, no. 6, pp. 23772387, 1988.

[186] M. Zhang, T. Desai, and M. Ferrari, "Proteins and cells on peg immobilized silicon surfaces," Biomaterials, vol. 19, no. 10, pp. 953-960, 1998.

[187] N. Khalilgharibi, J. Fouchard, N. Asadipour, A. Yonis, A. Harris, P. Mosaffa, Y. Fujita, A. Kabla, B. Baum, J. J. Munoz, M. Miodownik, and G. Charras, "Stress relaxation in epithelial monolayers is controlled by actomyosin," bioRxiv, 2018.

[188] D. Schneider, T. Baronsky, A. Pietuch, J. Rother, M. Oelkers, D. Fichtner, D. Wedlich, and A. Janshoff, "Tension monitoring during epithelial-tomesenchymal transition links the switch of phenotype to expression of moesin and cadherins in NMuMG cells," PLoS ONE, vol. 8, no. 12, 2013.

[189] A. Rigato, F. Rico, F. Eghiaian, M. Piel, and S. Scheuring, "Atomic force microscopy mechanical mapping of micropatterned cells shows adhesion geometrydependent mechanical response on local and global scales," ACS Nano, vol. 9, no. 6, pp. 5846-5856, 2015. 
Bibliography

[190] P. J. Sammak, L. E. Hinman, P. O. T. Tran, M. D. Sjaastad, and T. E. Machen, "How do injured cells communicate with the surviving cell monolayer?," Journal of Cell Science, 1997.

[191] V. E. Klepeis, A. Cornell-Bell, and V. Trinkaus-Randall, "Growth factors but not gap junctions play a role in injury-induced $\mathrm{Ca}^{2+}$ waves in epithelial cells," Journal of Cell Science, vol. 114, no. 23, pp. 4185-4195, 2001.

[192] S. Karsch, F. Buechau, T. M. Magin, and A. Janshoff, "An intact keratin network is crucial for mechanical integrity and barrier function in keratinocyte cell sheets," bioRxiv, p. 661462, 2019.

[193] C. Kröger, F. Loschke, N. Schwarz, R. Windoffer, R. E. Leube, and T. M. Magin, "Keratins control intercellular adhesion involving PKC- $\alpha$-mediated desmoplakin phosphorylation," Journal of Cell Biology, vol. 201, no. 5, pp. 681-692, 2013.

[194] J. Wegener, C. R. Keese, and I. Giaever, "Electric cell-substrate impedance sensing (ECIS) as a noninvasive means to monitor the kinetics of cell spreading to artificial surfaces," Experimental Cell Research, vol. 259, no. 1, pp. 158-166, 2000.

[195] M. Tarantola, A.-K. Marel, E. Sunnick, H. Adam, J. Wegener, and A. Janshoff, "Dynamics of human cancer cell lines monitored by electrical and acoustic fluctuation analysis," Integrative biology : quantitative biosciences from nano to macro, vol. 2, no. 2-3, pp. 139-150, 2010.

[196] S. Kagan, D. Ickowicz, M. Shmuel, Y. Altschuler, E. Sionov, M. Pitusi, A. Weiss, S. Farber, A. J. Domb, and I. Polacheck, "Toxicity mechanisms of amphotericin B and its neutralization by conjugation with arabinogalactan," Antimicrobial Agents and Chemotherapy, vol. 56, no. 11, pp. 5603-5611, 2012.

[197] S. F. Hsu and R. R. Burnette, "Characterization of the effects of amphotericin B on ion channels in MDCK cells using the patch-clamp technique," Biochimica et Biophysica Acta - Biomembranes, vol. 1329, no. 1, pp. 26-38, 1997.

[198] T. Yao and Y. Asayama, "Animal-cell culture media: history, characteristics, and current issues," Reproductive Medicine and Biology, vol. 16, no. 2, pp. 99-117, 2017. 
[199] P. S. Niranjan, J. G. Forbes, S. C. Greer, J. Dudowicz, K. F. Freed, and J. F. Douglas, "Thermodynamic regulation of actin polymerization," Journal of Chemical Physics, vol. 114, no. 24, pp. 10573-10576, 2001.

[200] X. Han, G. Li, G. Li, and K. Lin, "FTIR study of the thermal denaturation of $\alpha$ actinin in its lipid-free and dioleoylphosphatidylglycerol-bound states and the central and $\mathrm{N}$-terminal domains of $\alpha$-actinin in $\mathrm{D}_{2} \mathrm{O}$," Biochemistry, vol. 37, no. 30, pp. 10730-10737, 1998.

[201] D. J. Wright and P. Wilding, "Differential scanning calorimetric study of muscle and its proteins: Myosin and its subfragments," Journal of the Science of Food and Agriculture, vol. 35, no. 3, pp. 357-372, 1984.

[202] R. Waugh and E. A. Evans, "Thermoelasticity of red blood cell membrane," Biophysical Journal, vol. 26, no. 1, pp. 115-131, 1979.

[203] B. Liu, C. J. Goergen, and J. Y. Shao, "Effect of temperature on tether extraction, surface protrusion, and cortical tension of human neutrophils," Biophysical Journal, vol. 93, no. 8, pp. 2923-2933, 2007.

[204] F. Rico, C. Chu, M. H. Abdulreda, Y. Qin, and V. T. Moy, "Temperature modulation of integrin-mediated cell adhesion," Biophysical Journal, vol. 99, no. 5, pp. 1387-1396, 2010.

[205] R. Sunyer, X. Trepat, J. J. Fredberg, R. Farré, and D. Navajas, "The temperature dependence of cell mechanics measured by atomic force microscopy," Physical biology, vol. 6, no. 2, p. 025009, 2009.

[206] T. R. Kießling, R. Stange, J. A. Käs, and A. W. Fritsch, "Thermorheology of living cells - Impact of temperature variations on cell mechanics," New Journal of Physics, vol. 15, 2013.

[207] H. Nöding, "Active and passive microrheology of F-actin membrane composites," Dissertation, University of Göttingen, 2018.

[208] M.-C. Giocondi and C. Le Grimellec, "Temperature dependence of plasma membrane physical state in living Madin-Darby Canine Kidney cells," Biochemical and biophysical research communications, vol. 162, no. 3, pp. 1004-1009, 1989. 


\section{Bibliography}

[209] M. Théry, A. Pépin, E. Dressaire, Y. Chen, and M. Bornens, "Cell distribution of stress fibres in response to the geometry of the adhesive environment," Cell Motility and the Cytoskeleton, vol. 63, no. December 2005, pp. 341-355, 2006.

[210] S. Deguchi, A. C. Saito, T. S. Matsui, W. Huang, and M. Sato, "The opposite mechano-response of paxillin phosphorylation between subcellular and whole-cell levels is explained by a minimal model of cell-substrate adhesions," Journal of Biomechanical Science and Engineering, vol. 12, no. 2, pp. 1-12, 2017.

[211] Q. Tseng, E. Duchemin-Pelletier, a. Deshiere, M. Balland, H. Guillou, O. Filhol, and M. Thery, "Spatial organization of the extracellular matrix regulates cell-cell junction positioning," Proceedings of the National Academy of Sciences, vol. 109, no. 5, pp. 1506-1511, 2012.

[212] Y. Cai and M. P. Sheetz, "Force propagation across cells: mechanical coherence of dynamic cytoskeletons," Current Opinion in Cell Biology, vol. 21, pp. 47-50, feb 2009.

[213] R. Winklbauer, "Cell adhesion strength from cortical tension - an integration of concepts," Journal of Cell Science, vol. 128, no. 20, pp. 3687-3693, 2015.

[214] T. Lecuit and P.-F. Lenne, "Cell surface mechanics and the control of cell shape, tissue patterns and morphogenesis," Nature reviews. Molecular Cell Biology, vol. 8, no. 8, pp. 633-644, 2007.

[215] S. K. Wu, G. A. Gomez, M. Michael, S. Verma, H. L. Cox, J. G. Lefevre, R. G. Parton, N. A. Hamilton, Z. Neufeld, and A. S. Yap, "Cortical F-actin stabilization generates apical-lateral patterns of junctional contractility that integrate cells into epithelia," Nature Cell Biology, vol. 16, no. 2, pp. 167-78, 2014.

[216] A. F. Mertz, Y. Che, S. Banerjee, J. M. Goldstein, K. A. Rosowski, and S. F. Revilla, "Cadherin-based intercellular adhesions organize epithelial cell-matrix traction forces," Proceedings of the National Academy of Sciences, vol. 110, no. 3, pp. 842-847, 2013. 
[217] C. M. Nelson, D. M. Pirone, J. L. Tan, and C. S. Chen, "Vascular endothelialcadherin regulates cytoskeletal tension, cell spreading, and focal adhesions by stimulating RhoA," Molecular Biology of the Cell, vol. 15, pp. 2943-53, jun 2004.

[218] A. G. Clark, A. L. Miller, E. Vaughan, H.-Y. E. Yu, W. M. Bement, and R. Penkert, "Integration of single and multicellular wound responses," Current Biology, vol. 19, no. 16, pp. 1389-1395, 2009.

[219] P. O. T. Tran, L. E. Hinman, G. M. Unger, and P. J. Sammak, "A woundinduced $\left[\mathrm{Ca}^{2+}\right]_{i}$ increase and its transcriptional activation of immediate early genes is important in the regulation of motility," Experimental Cell Research, vol. 246, pp. 319-326, 1999.

[220] P. Wales, C. E. Schuberth, R. Aufschnaiter, J. Fels, I. García-Aguilar, A. Janning, C. P. Dlugos, M. Schäfer-Herte, C. Klingner, M. Wälte, J. Kuhlmann, E. Menis, L. Hockaday Kang, K. C. Maier, W. Hou, A. Russo, H. N. Higgs, H. Pavenstädt, T. Vogl, J. Roth, B. Qualmann, M. M. Kessels, D. E. Martin, B. Mulder, and R. Wedlich-Söldner, "Calcium-mediated actin reset (CaAR) mediates acute cell adaptations," eLife, vol. 5, pp. 1-31, 2016.

[221] R. Mège, J. Gavard, and M. Lambert, "Regulation of cell-cell junctions by the cytoskeleton," Current Opinion in Cell Biology, vol. 18, pp. 541-548, 2006.

[222] A. Abbott, "Biology's new dimension," Nature, vol. 424, pp. 870-872, 2003.

[223] M. Ravi, V. Paramesh, S. R. Kaviya, E. Anuradha, and F. D. Paul Solomon, "3D cell culture systems: advantages and applications," Journal of Cellular Physiology, vol. 230, no. 1, pp. 16-26, 2015.

[224] E. Cukierman, "Taking cell-matrix adhesions to the third dimension," Science, vol. 294, no. 5547, pp. 1708-1712, 2001. 

A. Appendix 


\section{A. Appendix}

\section{Mechanics during single-cell defects}
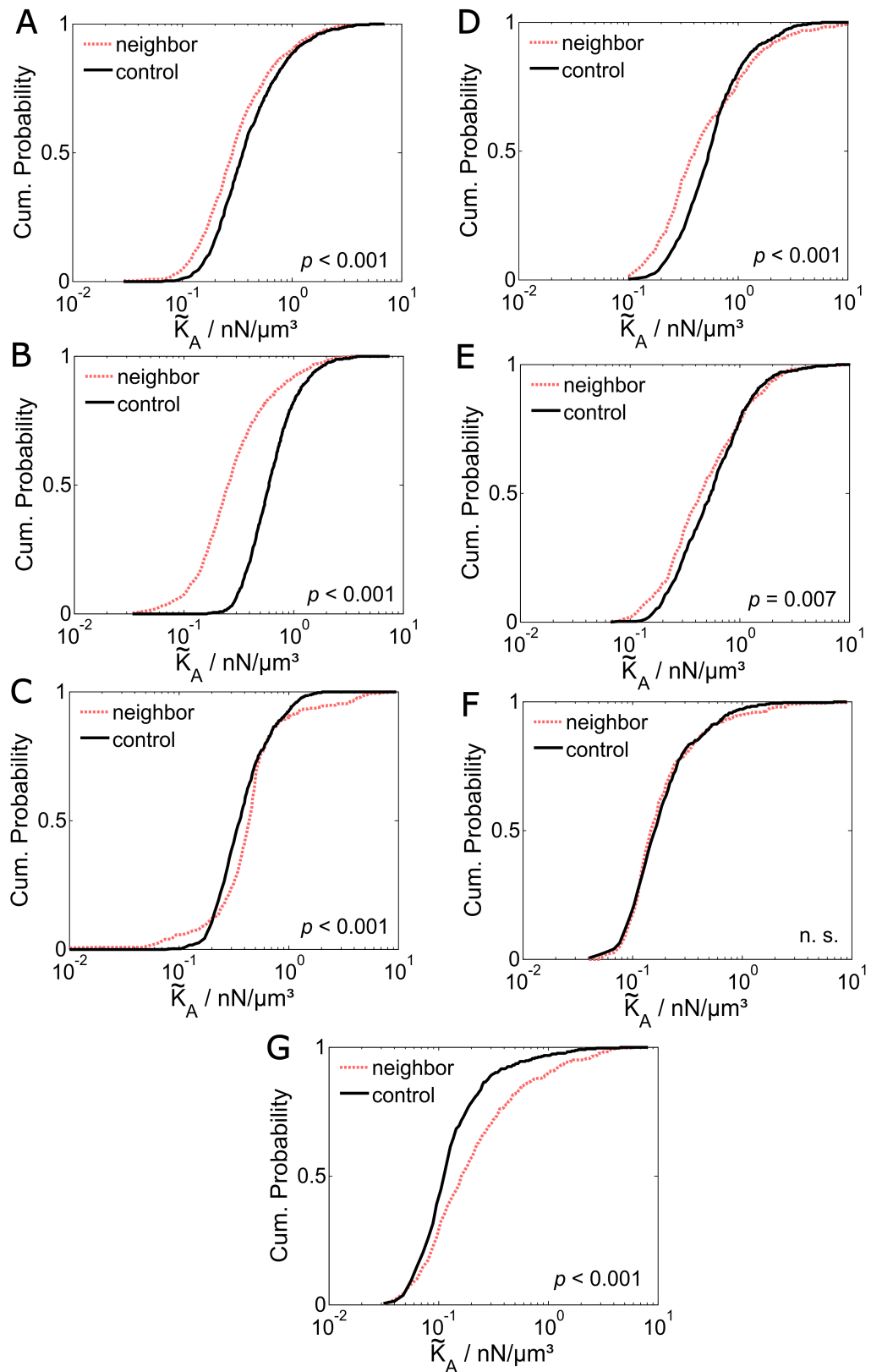

Figure A.1.: The apparent area compressibility modulus $\tilde{K}_{\mathrm{A}}$ does not consistently change in cells neighboring a single-cell defect (red, dashed) compared to cells in an undisturbed layer (black, solid) indicating that this parameter is not affected by this stimulus. Shown are the cumulative probabilities of 7 independent experiments. 

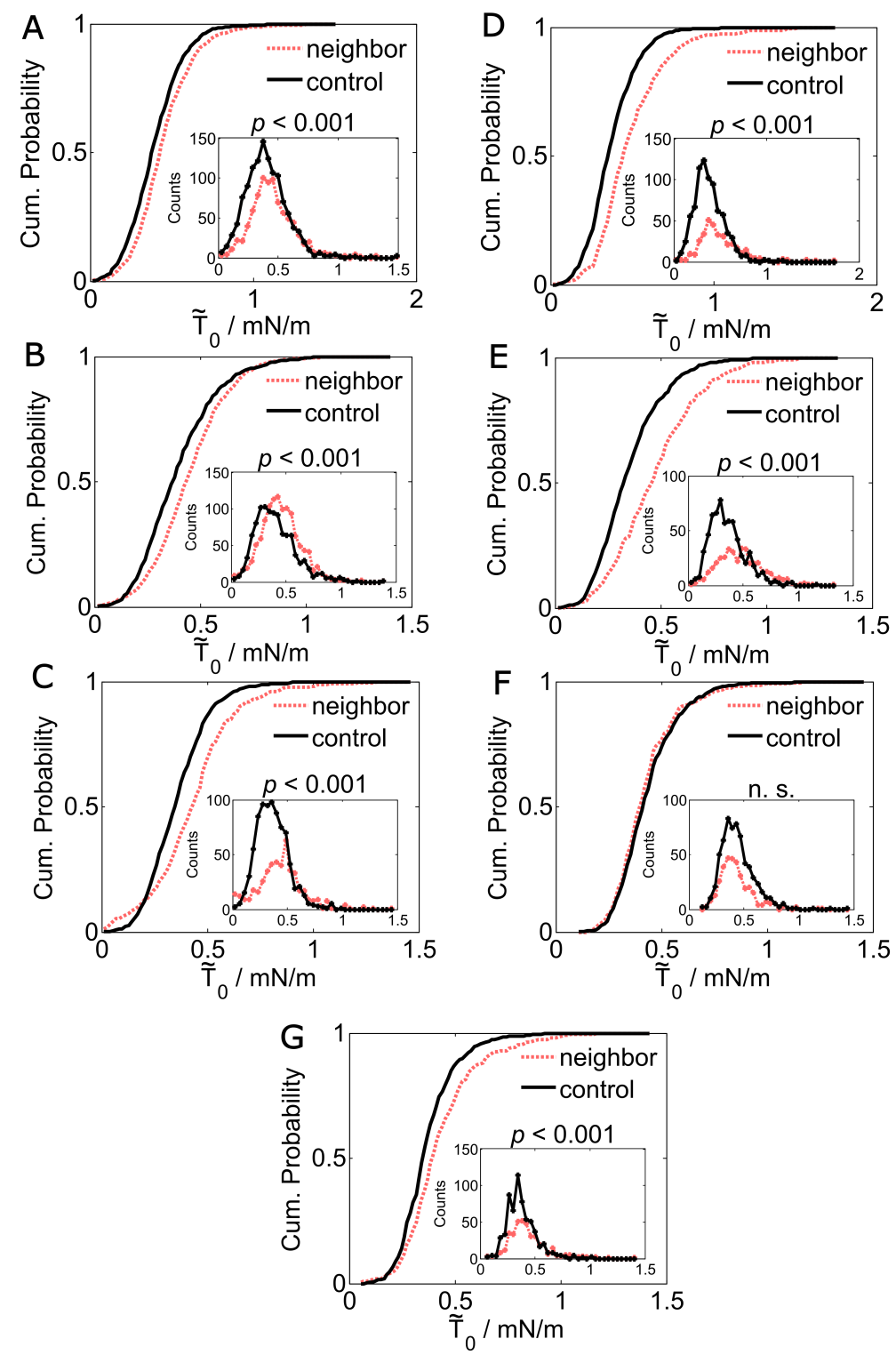

Figure A.2.: The apparent pretension $\tilde{T}_{0}$ shows a consistent change in cells neighboring a single-cell defect (red, dashed) compared to cells in an undisturbed layer (black, solid) indicating that this parameter is highly affected by this stimulus. Shown are the cumulative probabilities of 7 independent experiments and the histograms as inset. 


\section{A. Appendix}
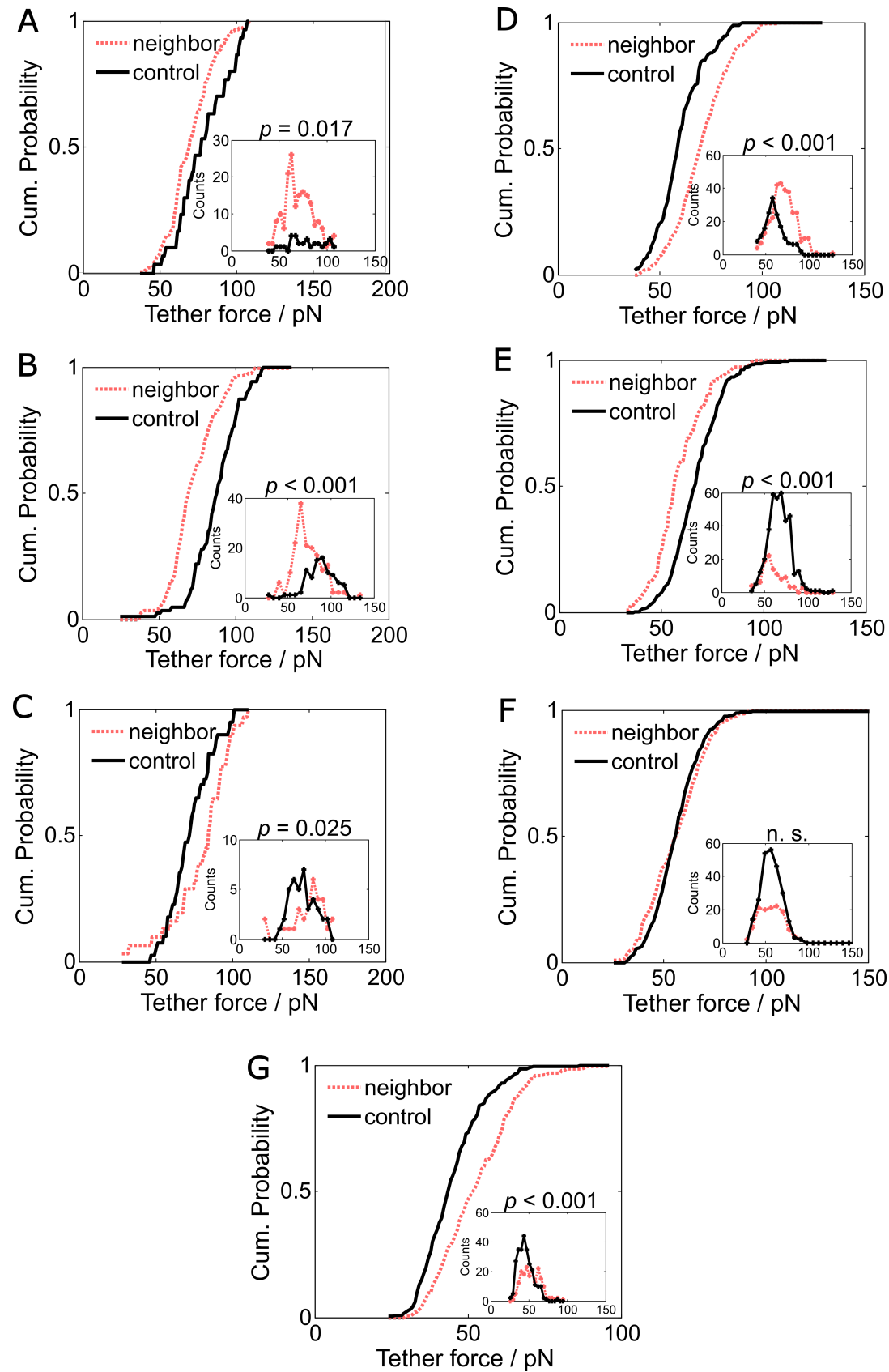

Figure A.3.: The tether rupture force does not consistently change in cells neighboring a single-cell defect (red, dashed) compared to cells in an undisturbed layer (black, solid) indicating that this parameter is not influenced by this stimulus. Shown are the cumulative probabilities of 7 independent experiments and the histograms as inset. 


\section{List of Figures}

1.1. An organism and its subunits . . . . . . . . . . . . . . . 2

1.2. An eukaryotic cell . . . . . . . . . . . . . . . . . . . . . . . 2

1.3. Geometry of epithelial cells . . . . . . . . . . . . . . . . . . . . 4

1.4. Cytoskeletal filaments inside the cell . . . . . . . . . . . . . . . . 5

1.5. Junctions at the cells' periphery . . . . . . . . . . . . . . . . . 9

1.6. Mechanosensing of cells . . . . . . . . . . . . . . . . . . . . 15

2.1. Methods used to create cell layers with large wounds . . . . . . . . . . . 29

2.2. Methods used to create single-cell defects . . . . . . . . . . . . . . . 30

2.3. Micro-patterning on a glass according to the protocol by Segerer et al. . 35

2.4. Probing the cells' mechanical properties with an AFM . . . . . . . . . . 38

2.5. A cone indenting a flat surface . . . . . . . . . . . . . . . . 40

2.6. The principle of electric cell-substrate impedance sensing . . . . . . . . . 42

2.7. The principle of cell adhesion noise spectroscopy . . . . . . . . . . . . . 43

3.1. Critical culture conditions during live cell experiments . . . . . . . . . . 46

3.2. Phase contrast images of MDCK II cells incubated for $2 \mathrm{~h}$ at different temperatures . . . . . . . . . . . . . . . . . . . . . . . . . . . . . . . . 48 48

3.3. Actin distribution inside MDCK II cells cultured for $2 \mathrm{~h}$ at different temperatures . . . . . . . . . . . . . . . . . . . . . . . . . . . . . . . . . . . 49

3.4. Ezrin distribution inside MDCK II cells cultured for $2 \mathrm{~h}$ at different temperatures . . . . . . . . . . . . . . . . . . . . . . . 49

3.5. Cell mechanics changes upon different temperatures . . . . . . . . . . 51

3.6. Heating efficiency in the used AFM compatible heating system . . . . . . 52

3.7. Formation of a MDCK II layer observed with cell adhesion noise spectroscopy . . . . . . . . . . . . . . . . . . . . . . 53 
3.8. Actin and E-cadherin distribution at different seeding densities and culture times . . . . . . . . . . . . . . . . . . 54

3.9. ZO-1 and actin distribution after 1 day of culture. . . . . . . . . . . 55

3.10. Apical polarity in cells with different seeding densities . . . . . . . . . 55

3.11. Actin distribution and cell height in densly seeded cells after 1 day and 2 days of culture . . . . . . . . . . . . . . . . . . 56

3.12. Actin filaments in densly seeded MDCK II after different culture times . . 57

3.13. Pattern obtained with the micro-patterning method . . . . . . . . 58

3.14. Isolated MDCK II cells on a micropattern . . . . . . . . . . . . . . . . 59

3.15. MDCK II cells with neighbor contact on a micropattern . . . . . . . . 59

3.16. Wound induction via classical wound healing essays. . . . . . . . . . . . 60

3.17. Closure of a single-cell defect . . . . . . . . . . . . . . . . 62

3.18. Pretension is altered in cells neighboring a defect . . . . . . . . . . . . 64

3.19. Mechanical investigation of the migration front of an incomplete cell layer 65

3.20. Cellular mechanics as a function of distance from the wound . . . . . . . 66

3.21. Neighbors of single-cell defects do not change their overall shape . . . . 67

3.22. Calcium signals after wounding . . . . . . . . . . . . . . . . . 69

3.23. Changes in junctional proteins during closure . . . . . . . . . . . . . 70

3.24. Contractility during wound closure . . . . . . . . . . . . . . . . . . . 71

3.25. Tight junction protein localization in keratinocyte cells . . . . . . . . . . 74

3.26. Adherens junction protein localization in keratinocyte cells. . . . . . . . . 74

3.27. Impaired junctions and fibrous structures in $\mathrm{Kty}^{-/-}$cells. . . . . . . . . . 75

3.28. Myosin localization in keratinocyte cells . . . . . . . . . . . . . . . 76

3.29. ECIS measurements reveal a barrier malfunction . . . . . . . . . . . . 77

3.30. Effects of changed cytoskeletal architecture . . . . . . . . . . . . . . . 79

3.31. Mechanics analyzed with the tension model in keratinocytes . . . . . . . 80

3.32. Cells adjacent to the site of single-cell manipulation respond differently to the micropipette wounding . . . . . . . . . . . . . . . . . . . . . 81

3.33. K14 rescue cells show a phenotype similar to WT cells. . . . . . . . . . . 83

3.34. K14 insertion partially restores barrier efficiency and mechanical integrity of cell monolayers . . . . . . . . . . . . . . . . . . . . . . . . . . . . . . . 84

4.1. Spatial extension of mechanical changes . . . . . . . . . . . . . . 93 
4.2. Schematic model of the wound response in keratinocytes . . . . . . . . . 97

5.1. Analyzing collective mechanical behavior in epithelial cells . . . . . . . 100

A.1. Apparent area compressibility modulus of cells neighboring a single-cell defect . . . . . . . . . . . . . . . . . . . 130

A.2. Apparent pretension of cells neighboring a single-cell defect . . . . . . 131

A.3. Tether rupture forces of cells neighboring a single-cell defect . . . . . . . 132 



\section{List of Tables}

2.1. Supplements in FAD medium for keratinocyte cell culture . . . . . . . . 26

2.2. Composition of LB media and agar solution used for plasmid amplification. 27

2.3. The antibody targeted structures with incubation time and concentration of the used antibodies . . . . . . . . . . . . . . . . . . . . . 31

2.4. The antibodies inculding dilution ratios used for experiments which are presented in section 3.5 



\section{Nomenclature}

\begin{tabular}{ll}
\hline Abbreviation & Long Name \\
\hline AFM & atomic force microscopy \\
Arp2/3 & actin related protein 2/3 \\
ATP & adenosine triphosphate \\
BSA & bovine serum albumin \\
ctrl & control \\
ECM & extra cellular matrix \\
FCS & fetal calf serum \\
DAPI & 4'6-diamidin-2-phenylindol \\
DMEM & Dulbecco's modifed eagle serum \\
DMSO & dimethylsulfoxide \\
DNA & deoxyribonucleic acid \\
ECIS & electrical cell-substrate impedance sensing \\
ECM & extracellular matrix \\
EDTA & ethylenediaminetetraacetic acid \\
ER & endoplasmatic reticulum \\
FA & focal adhesion \\
GFP & green fluorescent protein \\
GP & glycoprotein \\
FRET & Förster resonance energy transfer \\
HEPES & 4-(2-hydroxyethyl)-1-piperazineethanesulfonic acid \\
IF & intermediate filament \\
IgG & immunoglobulin G \\
K & keratin \\
Kty-/- & keratin type I knock out cells \\
&
\end{tabular}




\begin{tabular}{ll} 
LB medium & lysogeny broth medium \\
MDCK & Madin-Darby canine kidney \\
MT & microtubule \\
NA & numerical aperture \\
PBS & phosphate-buffered saline \\
PDMS & poly-(dimethylsiloxane) \\
PEG & poly(ethylene glycol) \\
PLL & poly(L-lysine) \\
P/S & penicillin/streptomycin \\
PSD & power spectral density \\
TBS & tris-buffered saline \\
TRITC & tetramethylrhodamine isothiocyanate \\
ULF & unit length filament \\
WT & wild type \\
$\mu P I P P$ & microscale plasma-initiated protein patterning \\
ZO & zonula occludens \\
\hline
\end{tabular}




\section{Danksagung}

So eine Arbeit kann nicht ohne die Unterstützung zahlreicher Menschen entstehen, denen ich an dieser Stelle danken möchte:

Zuerst möchte ich mich bei meinem Betreuer Prof. Andreas Janshoff bedanken, der mir die Möglichkeit gegeben hat, eine Doktorarbeit unter seiner Leitung anzufertigen. Während der Jahre hat er mich unterstützt und Anregungen eingebracht. Trotzdem wurden einem viel Freiheiten gelassen das Projekt selbst mit zu gestalten und den eigenen Weg durch die Promotion zu finden.

Außerdem möchte ich den weiteren Thesis-Komitee-Mitgliedern, Prof. Sarah Köster und Prof. Jörg Großhans, für die hilfreichen Diskussionen und Ratschläge danken. Ich habe mich immer sehr auf den regen Austausch in den Treffen gefreut. Zusätzlich danke ich den erweiterten Mitgliedern der Prüfungskommission, Prof. Silvio Rizzoli, Dr. Florian Rehfeldt und Dr. Thomas Burg, für die Bereitschaft meiner Disputation beizuwohnen und somit die Prüfung zu ermöglichen.

Meinen Kooperationspartnern Deqing Kong, Prof. Jörg Großhans, Dr. Fanny Büchau und Prof. Thomas M. Magin danke ich für die erfolgreiche Zusammenarbeit in den verschiedenen Projekten.

Für die Finanzierung während der Jahre danke ich der DFG und speziell dem Schwerpunktprogramm 1782 und dem Sonderforschungsbereich 937. Außerdem danke ich dem Promotionsprogramm GGNB und speziell den Mitarbeitern des Programms "Physics of Biological and Complex Systems", die einen mit Rat und Tat unterstützt und begleitet haben.

Aus meiner Arbeitsgruppe möchte ich zuerst denen danken, die viel zu oft vergessen werden. Unsere Zellkultur kann ich mir ohne Angela Rübeling nicht vorstellen. Danke, für all die Arbeit, die du mir abgenommen hast. Deine Unterstützung nicht nur bei Routine-Arbeiten, sondern auch bei Praktika und jeglichen Experimenten und Neuerungen, habe ich sehr wertgeschätzt. Ebenfalls danken für die zeitweilige Unterstützung 
in der Zellkultur möchte ich Anja Herdlitschke und Dr. Beate Rissom. Dr. Tabea Oswald als neue Mitarbeiterin möchte ich danken für die Unterstützung in der Zellkultur als auch für die hilfreichen fachlichen Diskussionen und ihr Engagement in organisatorischen Dingen. Außerdem möchte ich Petra Lappe für ihre offene Tür und ihre Unterstützung bei allen administrativen Angelegenheiten danken.

Von den Mit-Doktoranden haben besonders Helen Nöding, Thilo Baronsky und Andrea Cordes meinen Alltag geprägt. Sie haben mich immer fachlich sowie privat unterstützt und hatten jederzeit ein offenes Ohr. Die Gespräche tagsüber und auch abends haben mein Arbeitsumfeld sehr bereichert und mir stets weitergeholfen.

Fachlich unterstützt, besonders in der Anfangszeit, haben mich Bastian R. Brückner, Stefan Nehls und Ingo Mey. Dem gesamten AK Janshoff danke ich für die gemeinsame Zeit, ob in der Mensa, dem Kaffeeraum oder auch bei abendlichen Veranstaltungen und dem fachlichen Input über die Jahre hinweg. Außerdem möchte ich mich bei meinen Bachelor- und Master-Studenten bedanken, die zeitweise mit mir zusammen Experimente machten und ich somit auch von ihnen Neues lernen durfte.

Natürlich möchte ich auch Menschen aus meinem privaten Umfeld danken, ohne die ich diese Arbeit am Ende nicht verfasst hätte. Gute Freunde aus dem Physik-Studium, der Tennismannschaft oder anderen Bekanntenkreisen haben mich immer wieder aufgefangen, wenn ich Unterstützung brauchte. Gemeinsames Frühstücken, Kaffee trinken, Feiern, Tennis spielen, Segeln, Surfen, Sport gucken, Urlaub machen und überhaupt die vielen gemeinsamen Stunden an Wochenenden und Abenden waren wichtige Faktoren um auch mal abschalten zu können. Nur so konnte es danach motiviert weitergehen und diese Arbeit konnte entstehen. Rabea, dir danke ich zusätzlich für das Korrekturlesen der Arbeit und die aufmunternden Worte, die du immer bereit hattest.

Besonders möchte ich mich bei Markus bedanken, ohne den ich mir die finale Zeit dieser Doktorarbeit nicht vorstellen kann. Danke, dass du sie gemeinsam mit mir durchlebt und mich dabei so unglaublich unterstützt hast.

Abschließend möchte ich meiner Familie danken. Ihr habt den größten Dank verdient, weil ihr mich so treu begleitet und so viel Verständnis für mich und meine Arbeit aufbringt. Eure Worte haben mir immer wieder viel Kraft gegeben und mich ermutigt meinen Weg zu gehen. Trotzdem weiß ich euch jederzeit hinter mir, ihr seid die beste Unterstützung die man haben kann. Danke! 


\section{Curriculum Vitae}

\section{Personal Information}

Name: $\quad$ Susanne Karsch

Born: $\quad 8^{\text {th }}$ April 1990 in Nordhorn

Nationality: German

\section{Academic Studies}

since 02/2015 PhD student at the Institute of Physical Chemistry

within the group "Biophysical Chemistry"

University of Göttingen

10/2012 - 12/2014 Master of Science in Physics (grade 1.2)

University of Göttingen

Thesis: "Cytoskeletal Impact on Nuclear Shape and Mechanics in C2C12 Muscle Progenitor Cells" within the group "Cell \& Matrix mechanics" at the Third Institute of Physics - Biophysics

10/2009 - 09/2012 Bachelor of Science in Physics (grade 1.6)

University of Göttingen

Thesis: "Influence of Substrate Properties on Force Generation in Thrombocytes" within the group "Cellular Biophysics" at the Institute for X-ray Physics

\section{Education}

08/2002 - 06/2009 Hölty-Gymnasium Celle, Germany

General higher education entrance qualification (grade 1.6) 
08/1996 - 06/2002 Primary school and "Orientierungsstufe" in Waldshut and Hambühren, Germany

\section{Selected Conferences}

19th IUPAB congress and 11th EBSA congress, Edinburgh, UK

International Symposium "Biological Dynamics from Microscopic to Mesoscopic Scales", Grimma, Germany

\section{Publications}

- S. Karsch, D. Kong, J. Großhans, and A. Janshoff, "Single-cell defects cause a long-range mechanical response in a confluent epithelial cell layer", Biophysical Journal, vol. 113, no. 12, pp. 2601-2608, 2017.

- S. Karsch, F. Büchau, T. M. Magin and A. Janshoff, "An intact keratin network is crucial for mechanical integrity and barrier function in keratinocyte cell sheets", bioRxiv: 661462, 2019. 